\title{
TRAINING FOR VOLUNTEERS - A MULTIPLE CASE STUDY INVESTIGATING THE RELATIONSHIP BETWEEN VOLUNTEER MOTIVATIONS AND REQUIRED \\ TRAINING PROGRAMMES
}

BY

\section{PHILIP RONALD DALY}

\author{
A thesis \\ submitted to Victoria University of Wellington \\ in partial fulfilment of the requirements for a degree of \\ Master of Education
}

Victoria University of Wellington, New Zealand 


\begin{abstract}
This thesis reports on a multiple case study exploring the impact of required training programmes upon the motivations of new volunteers. The core research question is: How are the primary motivations of volunteers affected by the initial training that they are required to complete? Previous research has investigated the motivations of volunteers, and sought to measure the efficacy of volunteer training programmes. However there is little research investigating how volunteer motivations and volunteer training programmes influence each other.

Volunteers from two contrasting volunteer organisations were interviewed about their motivations to volunteer, experiences of the volunteer training, and any subsequent changes in motivation. Prior to these interviews the volunteers were given the Volunteers Function Inventory (VFI) questionnaire which measures volunteer motivations. The interview data was analysed using a six phase process of thematic analysis, and the motivational themes from this analysis were checked against the results of the VFI Questionnaire. The study found little difference in the overall motivations of volunteers between the two organisations, though there were differences in how these motivations were expressed within each organisation. Both sets of volunteers displayed high levels of altruistic and knowledge-based motivations, with the career motivation being the most variable, and age and stage of life appearing as a key moderator for the motivations. Volunteers appreciated training that they perceived as being relevant, and that utilised peer-learning, but both training programmes lacked the flexibility required to have a high level of relevance to a wide range of learning needs and motivations. Ultimately the motivations of the volunteers were improved by the training when they found that it either helped them to be more effective in their volunteer roles, or related well to their motivations for volunteering.
\end{abstract}




\section{Table of Contents}

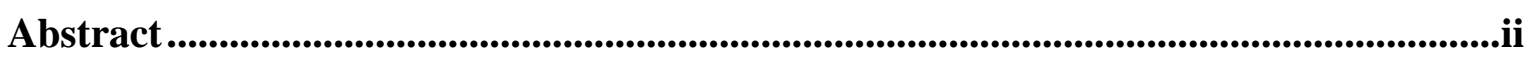

Table of Contents .......................................................................................................... iii

Acknowledgements.....................................................................................................................vi

Glossary ........................................................................................................................

List of Statistical Symbols ..................................................................................vii

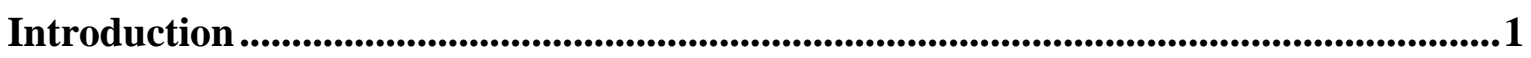

Personal Motivation for the Research ....................................................................

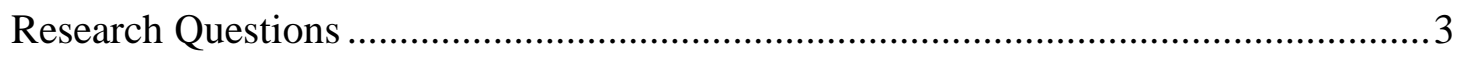

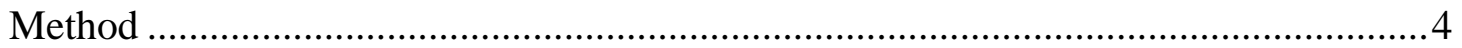

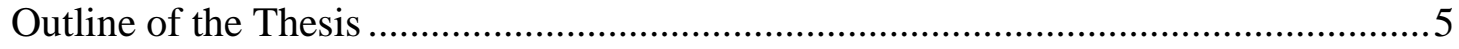

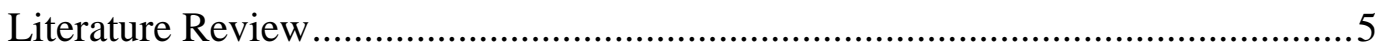

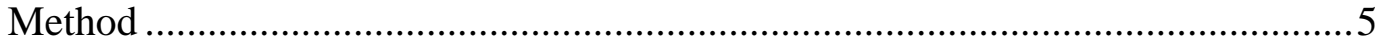

Results of the VFI Questionnaire .................................................................

Participant Perspectives from the Semi-Structured Interviews.................................

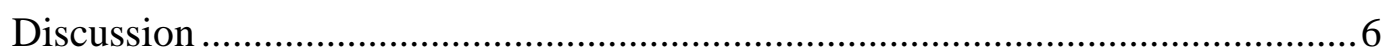

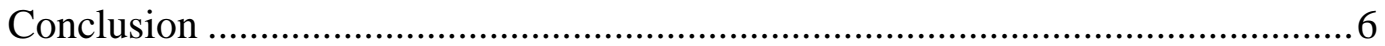

Literature Review..................................................................................................................

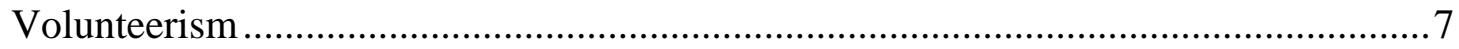

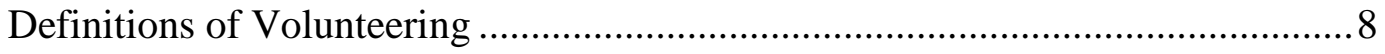

Differences across Culture and Nationality ..................................................... 10

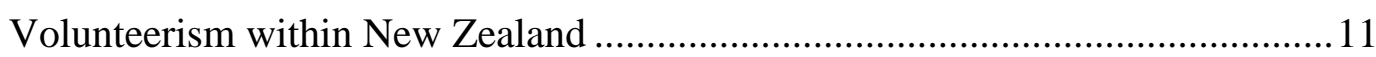

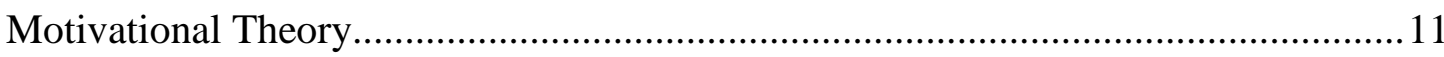

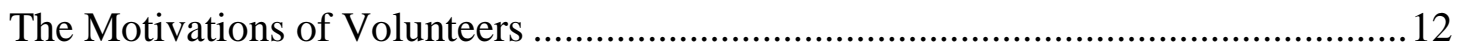

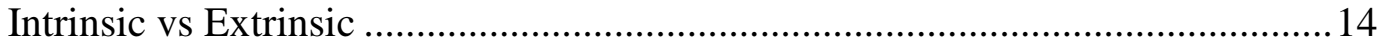

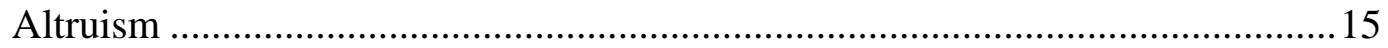

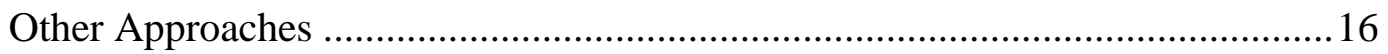

The VFI - A Functionalist Approach to Volunteer Motivation............................... 17

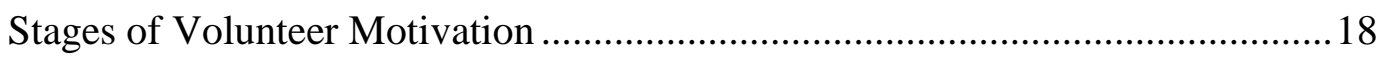

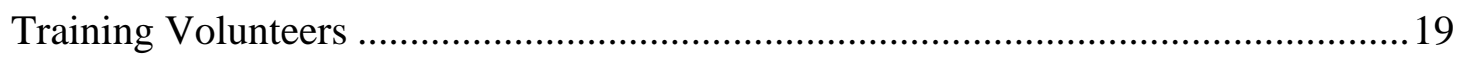

General Features of Effective Professional Development ....................................21

Professionalisation of the Volunteer Sector......................................................22

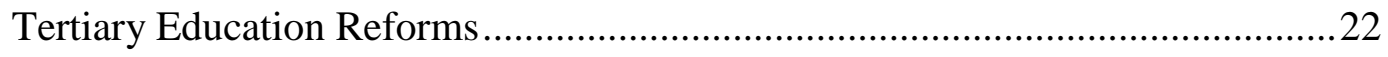

Method ..............................................................................................................................25

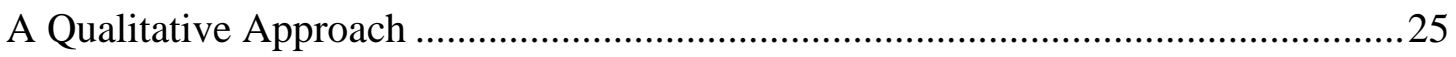

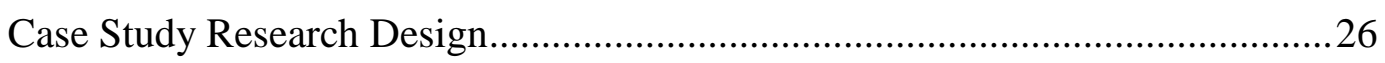

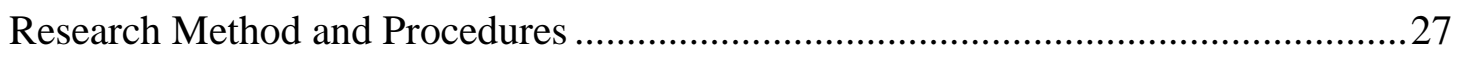




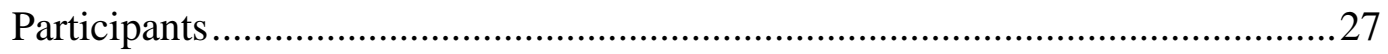

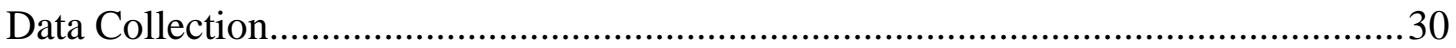

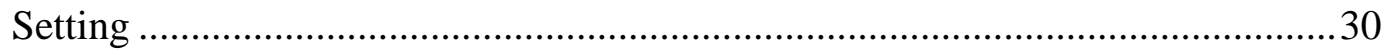

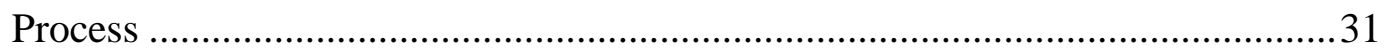

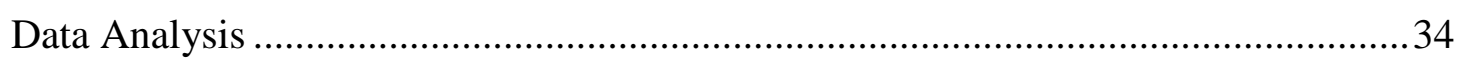

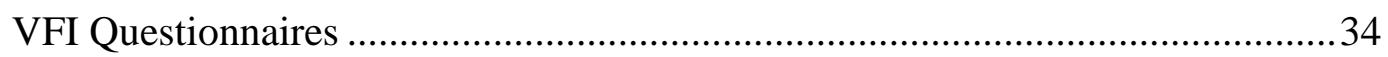

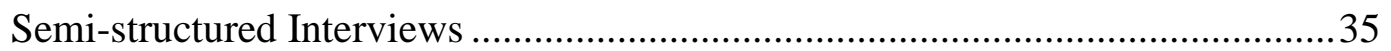

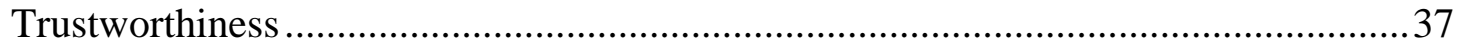

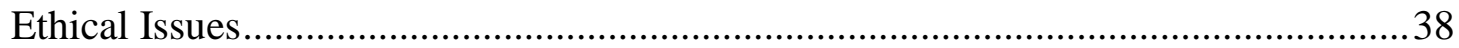

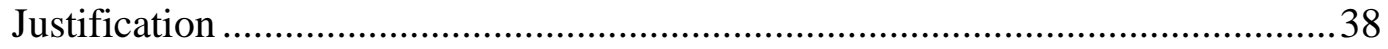

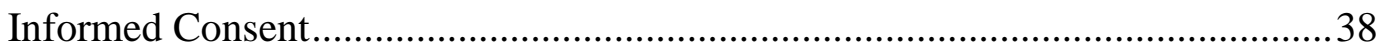

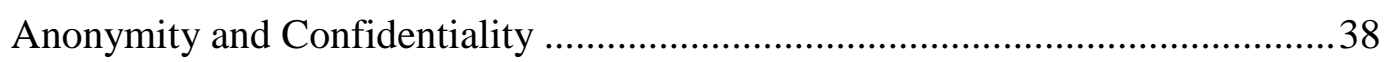

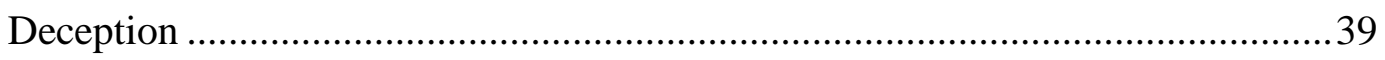

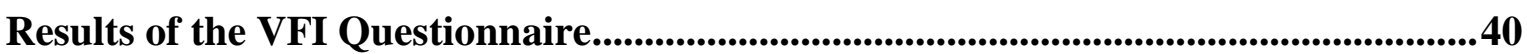

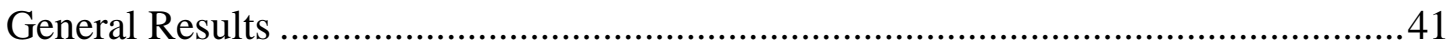

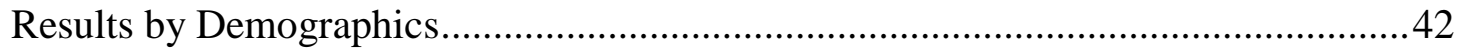

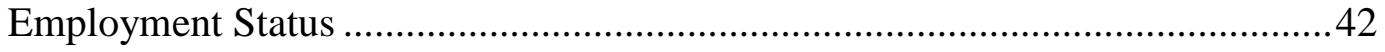

Age

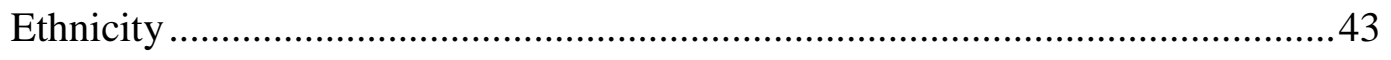

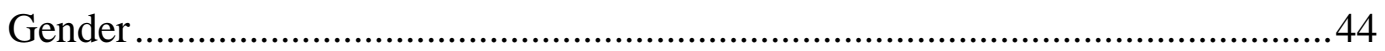

Participant Perspectives from the Semi-Structured Interviews ...................................46

The Motivations to Volunteer with Organisation A …...............................................46

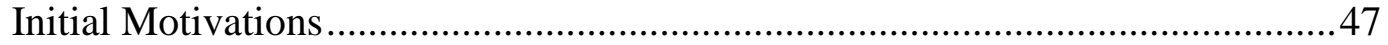

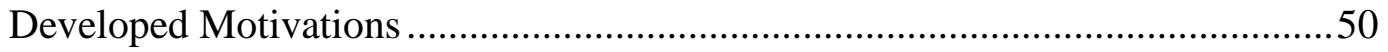

The Motivations to Volunteer with Organisation B................................................53

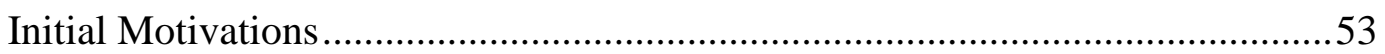

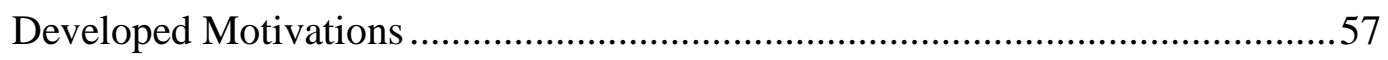

Analysis of Volunteer Motivations Using VFI Results .............................................59

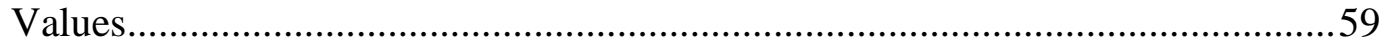

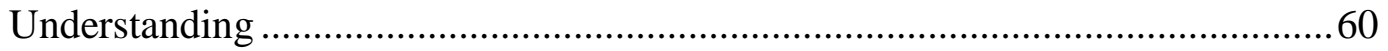

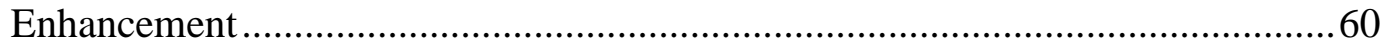

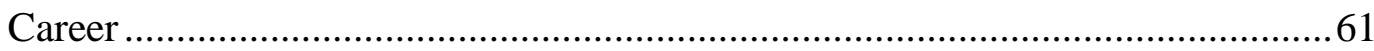

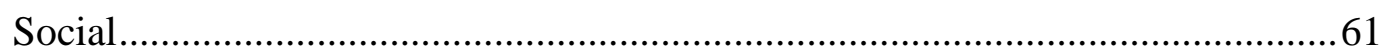

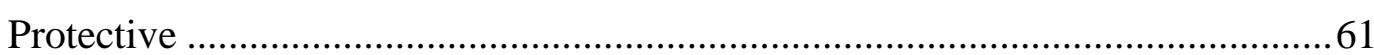

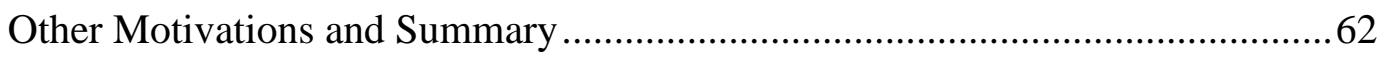

Volunteers' Perceptions of the Training Programme for Organisation A ...................62

Positive Aspects of the Training for Organisation A ...........................................63 
Negative Aspects of the Training for Organisation A ….....................................65

Volunteers' Perceptions of the Training Programme for Organisation B....................67

Positive Aspects of the Training for Organisation B ..........................................6 67

Negative Aspects of the Training for Organisation B........................................6 69

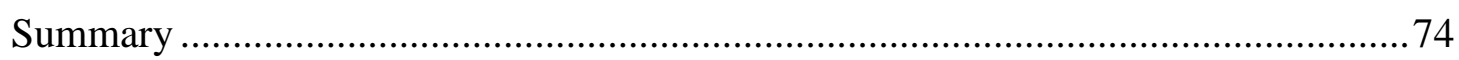

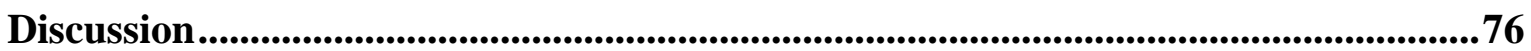

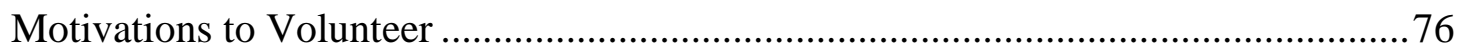

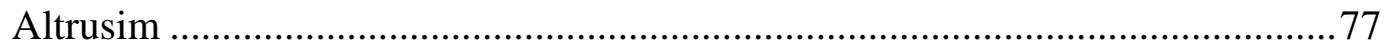

The Impacts of Age and Stage of Life ............................................................ 78

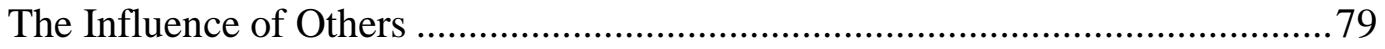

Loneliness and Other "Undesirable" Motivations ............................................... 80

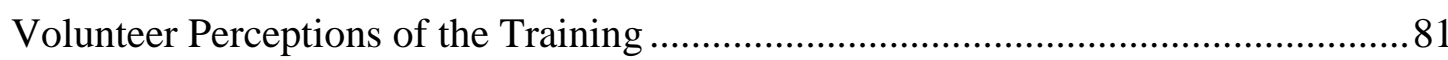

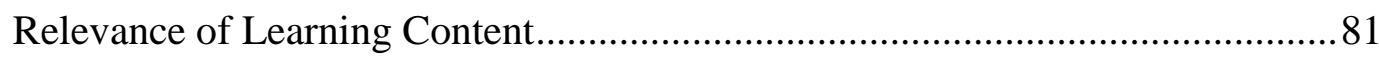

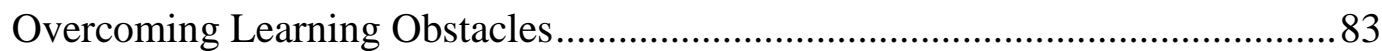

Individualisation and Recognition of Prior Knowledge and Skills...................... 84

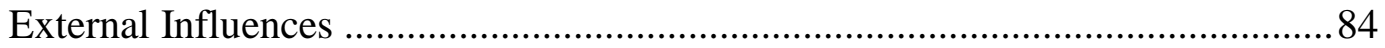

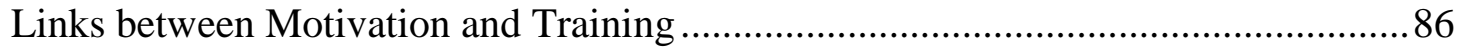

Factors that Enhanced Volunteer Motivation ....................................................... 86

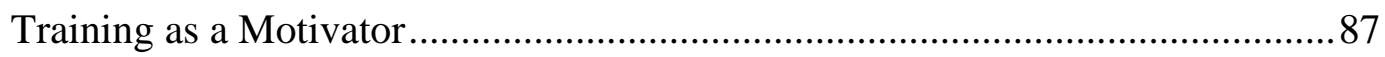

Training as a Potential De-Motivator............................................................ 88

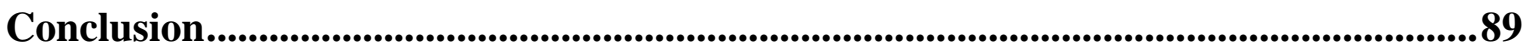

Recommendations for Volunteer Organisations ……............................................... 89

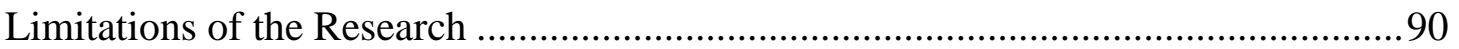

Recommendations for Further Research ............................................................ 91

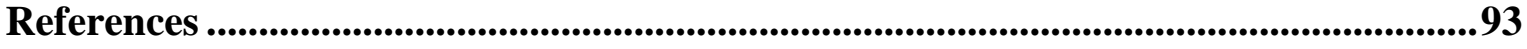

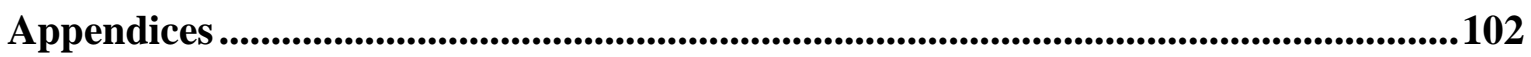

Appendix A - Organisational Information Sheet.................................................. 103

Appendix B - Organisational Consent Form ............................................................ 105

Appendix C - Participant Information Sheet .......................................................... 106

Appendix D - Participant Consent Form ................................................................ 108

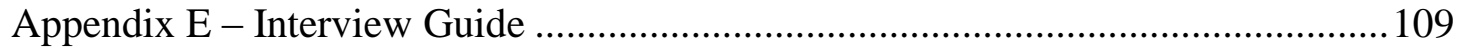

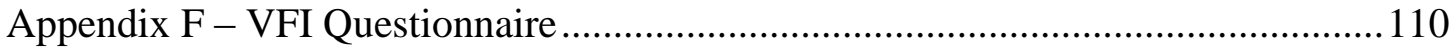

Appendix G - VFI Questions by Motivational Function........................................ 112 


\section{Acknowledgements}

The journey of writing this thesis has been a long and bumpy road, and a voyage that I would not have survived without the support of a number of special people. Though I cannot list them all, I do want to acknowledge the following:

Thank you to the seven volunteers, and two volunteer organisations, who agreed to participate in this research. I am inspired by your passion, and the volunteer work that you do is making this world a better place.

To my employer, The Salvation Army, and to all of my friends and colleagues at the Booth College of Mission, thank you. I could not have finished this thesis without your support: practically, financially, emotionally, and spiritually. In particular to Malcolm, for being a constant sounding board and source of encouragement and consolation. And to my various line managers - Caroline, Bryant and Liz, for your ongoing support and encouragement.

To all of the friends and family who have supported Tamsen, Mac, and myself through the last few years, and particularly through this last six weeks, thank you. Especially to those who accepted academic refugees (Lee and Bruce, Stew Collette and Micah, Clare and Nina, Lorraine, Leslie and Phil), to Mum and Dad for proofreading and meals, and to our wider families for the support and encouragement.

To my supervisor Dr Stephanie Doyle who guided me through a task which at times seemed unachievable. Thanks for your gentle encouragement, attention to detail, and well-timed suggestions.

And lastly, to Tamsen and Mac. We made it! Thanks for sticking in there, and allowing me the space and time to finish, particularly over the last few months. I love you both lots, but you should already know that. 


\section{Glossary}

National Certificate

NZQF

Pakeha

Te Tiriti o Waitangi

TEC

CALT

NCALNE

Unit Standards
A qualification that meets nationally endorsed standards and is listed on the NZQF (see below).

New Zealand Qualifications Framework. This framework contains all the secondary and tertiary qualifications that are nationally recognised and accredited.

A Māori term for non-Māori or New Zealanders who are of European descent.

The Treaty of Waitangi - New Zealand's founding document signed by the British Crown and Māori Chiefs. A good description of its content and history can be found at: https://nzhistory.govt.nz/politics/treaty-ofwaitangi

Tertiary Education Commission. A government agency charged with maintaining the tertiary education sector in New Zealand.

The Certificate in Adult Literacy Tutoring. An internal qualification (not on the NZQF) which was previously gained by tutors within Organisation B.

The National Certificate in Adult Literacy and Numeracy Education. The new qualification to be gained by tutors within Organisation B which is listed on the NZQF.

Units of learning that can be accrued towards a national qualification. Each unit standard has elements or learning outcomes which the learner must demonstrate in order to gain that standard.

\section{List of Statistical Symbols}

$M$

$N$

$n$

$S D$
Mean

Sample Size (full sample)

Sample Size (sub-sample)

Standard Deviation 


\section{Introduction}

No matter where you go in the world, in every country, city, and community, you will find volunteers; people who are utilising their time in the service of others, without any expectation of financial reward. There will, of course, be differences in how volunteerism is expressed in each of these contexts: some communities will have highly formalised volunteer structures, whereas for others it will be viewed as a natural part of living in community with others. In some volunteer environments there will be formal training systems, whereas for others it will be something that is learnt through experience and by watching others. Yet, despite these differences, volunteering is a common thread that can be traced across all cultures, religions, and nationalities.

Volunteerism is becoming an increasingly important factor within modern global economies. In the United Kingdom volunteers have been estimated to contribute $£ 40$ billion to the economy, and in Canada \$16 billion (Davis Smith, 2001). In a 2002 US study it was found that $44 \%$ of the adult population worked as a volunteer at some point during their lives, and $22 \%$ of the population were active in volunteering at that time (Lo Presti, 2013). In New Zealand it has been estimated that one third of the population is involved in some form of unpaid work for a voluntary organisation, with up to two thirds participating in any form of unpaid work outside of their home (Internal Affairs, 2016). These numbers could be even higher, with researchers suggesting that there may be widespread under-reporting of volunteer numbers. This may be due to the complexities of trying to define what might be considered volunteering, the challenges of trying to collect reliable data, and low response rates (Duxfield, 2016; Wilson, 2012).

The benefits of volunteerism are far greater than the economic benefits to organisations and countries. It is common for the majority of attention to be paid to the benefits for organisations and clients, with less attention paid to the benefits for volunteers, as well as the social (and non-financial) benefits towards society as a whole (Manetti, Bellucci, Como, \& Bagnoli, 2015). Volunteerism has been found to reduce mortality rates (Konrath, Fuhrel-Forbis, Lou, \& Brown, 2012; Wilson, 2012), enhance mental health (Wilson, 2012), and increase both a volunteer's job prospects and potential wage rate (Hackl, Halla, \& Pruckner, 2007; Wilson, 2012). A study by a 
New Zealand recruitment agency found that $93 \%$ of employers saw volunteering to be a credible form of real-work experience, and $89 \%$ believed that candidates who volunteered had an advantage securing employment (Volunteering New Zealand, n.d.). Socially, the benefits of volunteerism to communities are more than just economic, with correlations found between higher rates of volunteering and lower crime rates, lower levels of school absenteeism, and the breaking of stereotypes (e.g. for disabled volunteers) (Davis Smith, 2000).

As the importance of volunteerism has become more recognised, there has been a growing number of attempts to explore, and try to understand what the motivations of volunteers are. A better understanding of the motivations of volunteers can help organisations to recruit more effectively, and to retain them for longer. One of the most important studies in this area was conducted by Clary, Snyder, Ridge, et al. (1998), in which they developed the Volunteer Functions Inventory (VFI). Taking a functionalist approach, this inventory provides a framework to define the motivations of the volunteers according to six common functions that are served by volunteerism: values (altruistic and humanitarian concern for others), understanding (gaining knowledge, skills and abilities), social (engaging with others), career (as a means for career advancement), protective (to reduce feelings of guilt or negativity), and enhancement (to grow and develop one's ego).

There has been less research undertaken which aims to discover the most effective methods for training volunteers (Huang, Strawderman, Babski-Reeves, Ahmed, \& Salehi, 2014). Some attempts to describe best practices for volunteer training can be found, such as the Volunteer Training Framework developed by Volunteering South Australia (Deslandes \& Rogers, 2008), but there is little empirical research using rigorous research methods. Even less common is research which attempts to explore possible links between the two concepts of volunteer motivation and volunteer training (Newton, Becker, \& Bell, 2014). In the literature search for this thesis, there were just two studies found charting this relationship in any way (Newton et al., 2014; Pells, 2008b). It is this gap in the current literature that this research attempts to explore. 


\section{Personal Motivation for the Research}

The choice of this research topic has been influenced by both my present role and my upbringing. I am currently employed as a Project Manager at The Salvation Army, a large not-for-profit organisation that works with thousands of volunteers across New Zealand (and many more worldwide). In this role I have responsibility for coordinating training events for employees and volunteers of The Salvation Army nationwide, and working with other departments to create their own training resources. Additionally, as a child growing up within The Salvation Army, I witnessed many family members and role models doing volunteer work for this organisation. This included activities such as cleaning the church hall, working at the second hand clothes store, and running book sales. I too have been involved as a volunteer in many activities, including helping to run a youth group, leading a community gardening project, and living in a community drop-in house.

Because of this first-hand experience, at both a personal and professional level, I understand the vital role that volunteers play in our communities. Many of our notfor-profit organisations would simply not be able to function without volunteer labour. But I have also witnessed first-hand the growing professionalisation of many of these organisations, leading to increases in external compliance and regulations, and the lifting of expectations upon volunteers. In many cases this has led to positive change, with better resourced and safer work environments. But in some cases the balance has tipped, and the growing expectations have led to disillusionment, and volunteers leaving their roles. It is my hope that this research will help to start dialogue about how we can train volunteers in a way that inspires them in their service.

\section{Research Questions}

This research attempts to explore the motivations which compel people to volunteer, and how these are affected by the training that the volunteers are given. This is summarised by the main research question: How are the primary motivations of volunteers affected by the initial training that they are required to complete?

Most volunteer organisations require volunteers to undertake some form of training within the first year of volunteer service. If this training proves to be a demotivating 
factor for the volunteers the organisation could face high turnover rates. This would have wide-ranging impacts for the organisation including increased training and induction needs (due to high volunteer turnover), lack of institutional knowledge and experience, low morale, and loss of functionality. It is also beneficial for the volunteer that they receive training which they find motivating, and makes them more effective in their role. It has been widely stated that volunteers who are placed in roles that fulfil their motivations to volunteer, will be most satisfied, and willing to volunteer for longer (Clary \& Snyder, 1999; Douglas \& Rollins, 2007; Houle, Sagarin, \& Kaplan, 2005; Wilson, 2012). It follows that volunteers will be most likely to see training as a benefit to them if it relates positively to their motivations to volunteer (Newton et al., 2014; Pells, 2008b). Gaining a better understanding of the relationship between these two factors can be valuable in helping to shape training that is useful for both the volunteer and for volunteer organisations.

Sitting below the main research questions were three research sub-questions. What were the volunteers' primary motivations to volunteer? What were the volunteers' perceptions of the training that they are required to complete? Have the volunteers' motivations shifted since they started as a volunteer, either because of the training, or some other factor?

\section{Method}

As this is an area with little prior research a research method that allows for an exploratory approach was required. Qualitative research seeks to understand and describe people's experiences, which was a more fitting approach than a quantitative approach which typically seeks to test hypotheses or theories (Johnson \& Christensen, 2012). The use of case study allows for rich and detailed description, which further fits an exploratory approach, and two contrasting volunteer organisations were selected to provide contrasting perspectives. The use of case study fits research which is particularistic, descriptive and heuristic (Merriam, 2009).

Previous research into the motivations of volunteers has been criticised for lacking rigorous research methods (Shye, 2010). To protect against this potential limitation, rigorously tested research methods were selected for this study. The use of semistructured interviews was selected as the primary method of research, due to its 
applicability to both qualitative research, and the case study method. The data from these interviews were analysed utilising the six stage process of thematic analysis developed by Braun and Clarke (2006), and then the motivational themes were crosschecked against the results of the VFI Questionnaire. This VFI Questionnaire, as developed by Clary, Snyder, Ridge, et al. (1998), is one of the most rigorously tested methods of determining volunteer motivation.

\section{Outline of the Thesis}

\section{Literature Review}

The literature review contains a survey of current volunteer research, focussing on the aspects of motivation and training. Firstly the concept of volunteering is defined, differences across cultures and nationalities explored, before volunteerism within New Zealand is outlined. The broad topic of motivation is introduced next, and descriptions of various key approaches to the area of volunteer motivation are then described. Lastly, the training of volunteers is explored, including a brief summary of key aspects of professional development in the general workplace, and relevant recent changes within the New Zealand education sector.

\section{Method}

As noted above, this research utilises a qualitative case study approach. In the method chapter, this approach is explained, and the research participants are described. Data gathering methods, and the analysis of this data, is detailed, followed by an exploration of the trustworthiness of the research, and ethical considerations.

\section{Results of the VFI Questionnaire}

The next short chapter analyses the results of the VFI Questionnaire, including demographic breakdowns of the data.

\section{Participant Perspectives from the Semi-Structured Interviews}

The main aim of this section is to summarise interview responses according to the first two research questions: What were the volunteer's primary motivations to volunteer? What were the volunteer's perceptions of the training that they are required 
to complete? Firstly the motivations of the volunteers are examined, and findings are cross-checked against the VFI results from the previous chapter. Then the volunteers' perceptions of the training are analysed and described.

\section{Discussion}

Key themes from the research are discussed in the next chapter, as generated by the three research questions. Firstly themes about motivation are explored, including the nature of altruism, the impacts of age and stage of life, the influence of others, and loneliness and other undesirable motivations. Themes taken from responses about volunteer training are considered next, including the relevance of training, overcoming learning barriers, individualisation of training and recognition of prior skills, and outside influences. Lastly, the third research question is addressed, and links between training and motivation are considered. General factors that enhanced volunteer motivation are discussed, followed by aspects that made training to be a motivator, and then lastly aspects that made training to be a demotivating factor.

\section{Conclusion}

The research is summarised in the final section with reference to key findings and themes. Recommendations for volunteer organisations are presented, followed by the limitations of the research, and recommendations for future study. 


\section{Literature Review}

Before undertaking the empirical aspects of the research, an initial review of relevant literature was undertaken. This initial search utilised books and journal articles from Victoria University of Wellington's library, journal databases, and official volunteering websites. Discoveries from this initial literature search were used to shape the research questions and methodology for the research. Later, a more thorough and systematic literature search was undertaken to: get better depth of understanding in areas of importance, gain insight into areas that arose during the interviews, and ensure that the most recent relevant studies had been included.

This data is summarised below in four main sections. Firstly, volunteerism as a concept is considered and defined, with reference to differences across cultures and nationalities, and features of volunteerism within New Zealand. Motivation as a construct is then outlined, followed by a section exploring the motivations of volunteers. Within this section various key concepts are introduced, including the functionalist approach used by the VFI Questionnaire. Lastly, the training of volunteers is considered, factors which are seen as important in good professional development activities are detailed, and a description is provided of the New Zealand educational context within which this research was undertaken.

\section{Volunteerism}

Throughout the world, volunteerism plays an important role in every society and culture. As outlined in the previous section, the economic benefits of volunteerism are undeniable, and the volunteer sector plays a significant part in most economies around the world (Davis Smith, 2000). Because of this, governments around the world have attempted to harness and utilise the not-for-profit sector, including volunteers. This has led to fears that governments may eventually withdraw services, leaving the sector to pick up the pieces (Davis Smith, 2000; McDonald \& Warburton, 2001). The New Zealand Government has recognised the need to create an effective and collaborative relationship with the not-for-profit sector, including opportunities for meaningful involvement in decision-making (Internal Affairs, 2016; Senior, 2006).

Attempting to describe a typical volunteer is fraught with difficulty, given the wide breadth of activities, cultures, and nationalities involved. There are, however, some 
general characteristics that have been identified. Volunteers tend to score higher on the personality traits of extroversion and agreeableness compared to the general population, and, at least within the United States, they are more often women than men (Wilson, 2012). There is a higher rate of volunteerism amongst those with higher socio-economic status (Hustinx, Cnaan, \& Handy, 2010), as well as amongst those involved in part-time work (Wilson, 2012). Though there is a demand for volunteers in many areas, placing high recruitment demands on volunteer managers (de Haas, 2012; Pells, 2008a; Smith, Cordery, \& Dutton, 2010), this is not always the case. In a report investigating the demand for hospital volunteers, Handy and Srinivasan (2005) discovered that hospitals were not utilising all of the volunteers available to them, and considered any recruitment against whether the costs incurred by the volunteers would be offset by their output.

Whilst there is a growing body of research into volunteers and volunteerism, it is still a relatively under-researched area, particularly in studies utilising strong theoretical approaches (McDonald \& Warburton, 2001). A criticism of research in this field is the lack of strong theoretical frameworks (Shye, 2010), and the lack of a unified theory towards a phenomenon that spans multiple research fields, cultures, and nationalities (Hustinx et al., 2010). There is a high risk of bias in volunteerism research, with a preference for self-reporting increasing the risk of volunteers trying to make themselves look more favourable (Aydinli et al., 2016; Shye, 2010), and a general tendency across all literature to emphasise the more virtuous aspects of volunteerism (Hustinx et al., 2010).

\section{Definitions of Volunteering}

The United Nations definition of volunteerism underpins most common definitions around the world (see Ellis et al. (2014) for a comparison of the definitions across various European countries). This identifies three basic criteria for activities to be classified as volunteering, these being: not undertaken for financial gain, undertaken of one's own free will, and benefitting a third party or society at large (The Department of Internal Affairs, 2001). Within each of these criteria there is some flexibility, and none is mutually exclusive. For instance, an activity may be undertaken of one's own free will, yet it could still be influenced by feelings of obligation such as to society or culture. Snyder and Omoto (2008, p. 3) define 
volunteering as "freely chosen and deliberate helping activities that extend over time, are engaged in without expectation of reward or other compensation and often through formal organisations, and that are performed on behalf of causes or individuals who desire assistance." There is growing acknowledgement that due to the wide scope of volunteering activities across the world, any activity may only fall within the bounds of volunteering to a matter of degree (Cnaan, Handy, \& Wadsworth, 1996).

Other attempts to define volunteerism have emphasised the nature of the activity, or the levels of engagement of the volunteer. Examinations of the nature of the activity have included the difference between planned and spontaneous acts of helping (Aydinli et al., 2014), the length of service, ranging from episodic events through to continuous service (Mykletun \& Himanen, 2016), and the nature of volunteerism, such as mutual aid, philanthropy, participation and advocacy (Davis Smith, 2000). Descriptions of the levels of engagement of volunteers have included willingness to engage, ranging from obligated to enthusiasts (Alexander, Kim, \& Kim, 2015), and levels of enthusiasm, ranging from active engagement through to passive involvement (Holmes \& Slater, 2012). A model developed by Snyder and Omoto (2008) proposes volunteerism as a process with three stages: antecedents, experiences and consequences, and across four levels: the individual, interpersonal, agency and societal. Antecedents refer to the personality, motivational, and circumstantial characteristics of individuals that lead them to volunteer. Experiences refer to the psychological and behavioural aspects of interpersonal relationships that occur whilst volunteering, and consequences refer to the impact of their service at various levels.

An area of volunteering that is attracting increasing attention is informal volunteering. The distinction between formal and informal volunteering is constantly moving, but historically formal volunteering has been described as taking place within a non-profit organisation, and in a designated volunteer position (Ellis et al., 2014). By contrast “informal volunteers are self-appointed and self-managed, engaging in activities such as helping neighbours, supervising local traffic flow, or sending information to politicians" (Hustinx et al., 2010, p. 7). In Western cultures formal expressions of volunteerism are more common, whereas among minority groups, less developed countries, and lower socio-economic groups, informal volunteering is more frequent (Hustinx et al., 2010). The increasing recognition of informal volunteer activities 
prompted Volunteering Australia to re-examine, and ultimately change their definition of volunteering, to be more inclusive of these activities (Ellis et al., 2014).

\section{Differences across Culture and Nationality}

Volunteerism, in some form, occurs within every country of the world, though how it is expressed varies widely across different cultures, groups, and nationalities. A study of business students in Europe and the United States by Offstein, Chory, \& Childers Jr (2015), demonstrated differing approaches to a volunteering task, highlighting different understandings of volunteerism between the two cultures. When the two groups of students were given loose instructions to accomplish "five hours of goodness" that would "make the world, the community, the life of another better" the US students chose to undertake the volunteer service individually and in areas where they were already active, whereas the Central European group chose to collaborate on a shared project that they created together as a result of the project (Offstein et al., 2015, p. 77). This difference of approach may not only suggest a difference in culture and understanding between the two groups, but also a wider shift in the nature of volunteerism. The modernisation of society emphasises the importance of individuality, which in turn appears to be shifting volunteerism in some parts of the world towards a more individualistic approach (Wilson, 2012). Davis Smith (2000) identifies widespread differences in the public perceptions of volunteering between countries, noting that the less economically developed the country is, the less formal its volunteering structures are likely to be. Countries with less structured volunteering networks therefore rely more heavily on informal networks of mutual aid and selfhelp.

Motivations can be strongly affected by culture, with understanding of volunteerism differing across various cultures. Within New Zealand, for example, the term volunteer is rarely used by Māori and Pacific peoples, who see many of these activities as an expected contribution to their community and/or family (Tamasese, Parsons, Sullivan, \& Waldegrave, 2010; The Department of Internal Affairs, 2001). Within these cultures, the common Western motivation of altruism is replaced by notions of obligation, duty, reciprocity and mutuality (Tamasese et al., 2010). For Māori, the term manaakitanga is preferred to volunteerism, which is loosely translated as hospitality, and denotes acts of caring and sharing with others (The Department of 
Internal Affairs, 2001). The notion of volunteering as a normal part of community life is also noted within religious communities, where it is commonly expected that church members will contribute both to their church and the wider community (Lukka \& Locke, 2003).

\section{Volunteerism within New Zealand}

Volunteering New Zealand defines volunteering as "work done of one's own free will, unpaid, for the common good" (Volunteering New Zealand, 2014, p. 1). Rates of volunteerism and giving within New Zealand are amongst the highest in the world, with it being ranked as the world's fourth most charitable country in the 2016 World Giving Index (Internal Affairs, 2016). The labour value of volunteers has been estimated at $\$ 3.5$ billion per year, which equates to $1.7 \%$ of New Zealand's Gross Domestic Profit. A national survey in 2013 found that the number of people volunteering had risen by $20 \%$; however, the average hours that a volunteer worked had fallen (Internal Affairs, 2016).

As noted earlier, there has been an increased willingness from the New Zealand Government to partner with volunteer organisations in the not-for-profit sector. Historically this has not always been the case, with the 20th century seeing a "moving frontier" of welfare between the not-for-profit sector and the state (Tennant, 2001). The rise of the New Zealand welfare system in the 1970s was in many ways a state response to the failure of the voluntary sector to provide for the public's welfare needs. Today, however, the state and not-for-profit sector are closely linked together, with Government grants totalling $\$ 4.1$ billion pouring into the sector, representing $10.8 \%$ of total income (Internal Affairs, 2016). But this relationship is not totally welcomed by all. Many within the not-for-profit sector remain wary of the increasing compliance and regulatory requirements that come with government funding (Ellis et al., 2014; Internal Affairs, 2016).

\section{Motivational Theory}

Motivation has been conceptualised in various ways over the history of motivational research. Early theories were based around the belief that motivations were created by inherent factors within the individual such as drives, needs and beliefs (Urdan \& Schoenfelder, 2006). Later behaviourist theories, such as Skinner's operant 
conditioning approach, proposed that motivation to engage in learning comes from contingencies associated with familiar tasks (Skinner, 1954). Thus, positive reinforcement will encourage a learner to attempt similar tasks, but negative reinforcement will reduce future motivation for similar tasks. Cognitive approaches focus on a learner's belief about the probability of success in a learning activity. An example of this cognitive approach are attribution theories, which state that people try to bring order to their lives by developing implicit theories as to why things have happened (Tollefson, 2000). Social cognitive theories, such as Bandura's theory of self-efficacy, state that individuals' beliefs are also influenced by social contextual factors. In his theory of self-efficacy, Bandura (1986) argued that an individual's belief as to whether they could achieve a particular task is the most crucial determinant as to whether they will go on to complete it.

Other approaches include those focussed upon personal agency beliefs such as selfdetermination theory (Deci \& Ryan, 2008). In this theory Deci and Ryan propose that humans have three innate needs which must be fulfilled in order to achieve selfmotivation and personal well-being. These needs are: relatedness (the ability to find security and belonging in a social environment), competence (developing self-worth by experiencing mastery) and autonomy (to have individual control over one's own life and actions). In his motivational systems theory, Ford (1992) proposes that achievement and competence are the results of a motivated, skilful and biologically capable person interacting with a responsive environment. Thus the four prerequisites for someone to complete an activity effectively are: the person must have the motivation to initiate and maintain the activity until it is complete, the person must have the skill necessary to execute the activity, the person's biology must be able to support the operation of the motivation and skill components, and the person must have a cooperative environment.

\section{The Motivations of Volunteers}

People have various motivations for volunteering which are often complex, multilayered, and can develop and change over time (Clary \& Snyder, 1999; Pells, 2008b; Yeung, 2004). Further, individuals may not be consciously aware of their true motivations for volunteering (Aydinli et al., 2014; Offstein et al., 2015). Motivational differences have been discovered across various nationalities, which suggest that they 
may be affected by both structural factors, such as political and welfare systems, as well as cultural factors, such as the levels of individualism and religiosity (Grönlund et al., 2011). Educational achievement has been demonstrated to be the strongest predictor as to whether someone will volunteer or not (at least in a formal capacity), with those with higher education being the most likely to volunteer (Burns, Reid, Toncar, Anderson, \& Wells, 2008; Shye, 2010; Wilson, 2012). Getting people to volunteer early in life has been shown to be a powerful tool to enhance volunteerism, with studies showing that those who volunteer in high school (even when obligated) are more likely to continue to volunteer in college and then also into their working life (Fényes, 2015; Simha, Topuzova, \& Albert, 2011). In a study of volunteers working at a healthy living centre, Warren and Garthwaite (2015) noted that many of those volunteering decided to either enter or re-enter volunteerism as a result of a milestone event in their life such as their children leaving home, or retirement. However, it has been found that retirees who have no previous history of volunteerism are very unlikely to start volunteering once they retire (Wilson, 2012).

One area receiving particular attention in the area of volunteer motivation, is the influence of religion and spirituality. Youth involvement in religion has been found to have a positive effect on volunteerism as an adult, and is a stronger predictor than gender, marital status or nationality (Wilson, 2012). Okun, O'Rourke, Keller, Johnson, and Enders (2015) found that religiosity (a pursuit of the sacred via membership in religious institutions) was a stronger predictor than spirituality (a reference to transcendence without necessarily being bound to religious traditions). In a study of youth in Eastern Europe, Fényes (2015) found that church attendance alone was a weak predictor of volunteer participation; however, it became significant when isolated to those who regularly prayed, or belonged to religious sub-groups (such as a youth group). Lukka and Locke (2003) note that it is dangerous to categorise faith based volunteering as a single entity, as each faith has distinctive features, shaped by the belief systems, norms, and institutions of the faith community which shape the experiences of volunteers within that faith. In their study of various faith groups in England, they did, however, identify certain principles which guided all of the groups, such as acting selflessly, helping those who are needy, and being concerned with social justice and inequality. 
There have been numerous attempts to define and categorise the motivations of volunteers. The most common approach has been to split motivations into distinct categories such as intrinsic and extrinsic, or altruistic and self-serving, as well as many others that have multiple categories. Alternative approaches have drawn upon wider motivational concepts such as role identity theory (Callero, Howard, \& Piliavin, 1987; Chacón, Vecina, \& Dávila, 2007; Piliavin, Grube, \& Callero, 2002) and attachment theory (Erez, Mikulincer, van Ijzendoorn, \& Kroonenberg, 2008).

Whilst it would be impossible to detail all of these approaches, some of the most important distinctions are listed below.

\section{Intrinsic vs Extrinsic}

A common definition of intrinsic motivation is that a person "is intrinsically motivated to perform an activity when one receives no apparent reward except the activity itself" (Deci, 1971, p. 105). By contrast, extrinsically motivated activities are performed to obtain a separable outcome (Finkelstien, 2009), and thus are driven by external rewards, such as the desire to improve human capital, social recognition, or physical rewards (Degli Antoni, 2009).

Whilst intrinsic and extrinsic motivational factors are both powerful determinants of human behaviour, they have differing effects on volunteering behaviour (Cappellari \& Turati, 2004). A survey of data from employees at an Italian public utility company by Cappellari and Turati (2004) found that those who had higher levels of intrinsic motivation were more likely to volunteer than those who were more extrinsically motivated. In a study of US undergraduate students, Finkelstien (2009) found that those driven by intrinsic motivations had a correlation with prosocial personalities and the establishment of volunteer role identities (the process whereby a volunteer internalises the role of volunteer), whereas there was no link for either of these constructs from those driven by extrinsic motivations. Surprisingly, in this study motivational orientation did not have a significant effect on either the length of time spent volunteering, or the strength of commitment shown by the volunteer. 


\section{Altruism}

Chou (1996, p. 297) defines altruism as "voluntary, intentional behaviour motivated to benefit another that is not motivated by the expectation of external rewards or avoiding externally produced punishments or aversive stimuli”.

There has been a long history of debate as to whether true altruism exists, or whether any act thought to be of an altruistic nature actually serves deeper, and possibly hidden, selfish motives (for example to enhance social standing or feel better about oneself) (Burns, Reid, Toncar, Fawcett, \& Anderson, 2006; Clary, Snyder, \& Stukas, 1998). The consensus seems to have shifted to the point of view that true altruism may indeed exist as a motive (Piliavin \& Charng, 1990). Clary, Snyder, \& Stukas (1998) note that true altruism may not actually be a useful notion to aim for, as it can be linked to issues with volunteer burnout, and unrealistic expectations which alienate potential volunteers. A more helpful way to look at it, may be as a conceptual duality of altruistic and self-serving motivations, where the volunteer sits somewhere between the two (Shye, 2010). There are always a number of motivational factors evident for any volunteer, and thus there will exist a complex interaction between altruistic and self-serving factors (Clary, Snyder, \& Stukas, 1998; Cnaan \& GoldbergGlen, 1991; Hustinx et al., 2010). In a study of marketing students from five US universities, Burns et al., (2006) discovered that rather than appearing as a separate motive, altruism was present as a factor in all of the other motivations noted by the students as reasons to volunteer.

Whilst volunteers may be motivated by a combination of altruistic and self-serving motivations, certain factors will usually be felt more strongly than others. In a study of 13 countries, Grönlund et al. (2011) found that across all of the countries there were connections between individualism and resume motivations (i.e. trying to improve job prospects) and egalitarianism and altruistic motivations. Okun et al. (2015) found that volunteers with a high altruistic motivation had an increased likelihood of volunteering, and that this was even more pronounced when combined with high levels of religiosity. In fact, altruism, or other terminologies with a similar meaning, has been measured as the most important factor in the majority of studies, across a wide variety of volunteer service, and volunteer delineations (Pells, 2008a). Some examples include the following areas: those over 50 (Principi, Chiatti, \& 
Lamura, 2012), Generation Y (Burns et al., 2008), bike race volunteers (Mykletun \& Himanen, 2016), corporate social responsibility (Do Paço, Agostinho, \& Nave, 2013), organisations caring for people with AIDS (Stewart \& Weinstein, 1997), palliative care (Muckaden \& Pandya, 2016), cancer education (Jasperse, Herst, \& Kane, 2012), firefighters (de Haas, 2012), and conservation workers (Douglas \& Rollins, 2007). These results need to be interpreted with a degree of caution, however. Due to the self-reported nature of all of these studies, the prospect of reporting bias poses a high threat to validity. There is a distinct possibility that respondents will tend to report motivations that they believe will make them look better, even if this occurs at a subconscious level (Aydinli et al., 2016; Shye, 2010).

\section{Other Approaches}

Other research has defined volunteer motivation in different ways. Hackl et al. (2007) borrowed from economic theory and split motivation into a consumption group, which was defined as those undertaking utility bearing activities to help others, and an investment group, containing those who were interested in building human capital. In a literature review Pells (2008a) noted three main categories that volunteer motivations tended to be split into: consumptive, investment and altruistic. A unique approach put forward by Yeung (2004), is the octagon model of volunteer motivation. This model was devised as a result of a phenomenological study of volunteers from the Evangelical Lutheran Church of Finland, and maps four dimensions, each with two contrasting poles which are either inward or outward focused. The eight poles are getting and giving, continuity and newness, distance and proximity, and thought and action. Yeung contends that volunteers will sit somewhere in the middle of each of these poles, making it possible to map out a complex motivational profile of the volunteer.

Cnaan and Goldberg-Glen (1991) argue that two, three, or even six category models are insufficient, and that volunteer motivation can only be described as a unidimensional model. Through examination of all meaningful previous studies of volunteer motivation, they mapped out 22 distinct motives which could not easily be placed within any of the previous 2, 3, or 6 category models. Thus, they concluded that volunteers do not actually distinguish between types of motives, but rather act on a unique combination of any of the various motives that they had outlined. This 
combination of motives comes together for the volunteer as a unified whole, which they have termed a unidimensional model.

Several studies have proposed new motivations, specific to their particular context, which have not been covered within previous motivational frameworks. These include an adrenalin surge for emergency services volunteers (Pells, 2008b), the myth of a hero's adventure for overseas development workers (Hudson \& Inkson, 2006), and the attraction of a foreign destination for volunteer tourists (Grimm \& Needham, 2012).

\section{The VFI - A Functionalist Approach to Volunteer Motivation}

The most common method for measuring volunteer motivation is the Volunteer Functions Inventory (VFI) which was developed by Clary, Snyder, Ridge, et al. (1998). Functional theories of motivation describe the adaptive and purposeful strivings of individuals towards personal goals, and emphasise that similar behaviour can serve different psychological functions for different people (Clary, Snyder, Ridge, et al., 1998; Houle et al., 2005). Clary, Snyder, Ridge, et al. (1998) stress that volunteers may have a combination of any of these motivations, and two volunteers in the same organisation will likely have a completely different combination of motivations even when undertaking similar tasks. Further, two people can have similar purposes for volunteering which serve completely different motivational functions. For example, two volunteers could state that they enjoy the interaction with experienced peers whilst serving as a volunteer. However, whilst Volunteer A may enjoy this simply because of the social interaction, Volunteer B may enjoy the wisdom the experienced volunteer imparts, which enables them to complete assignments towards a desired qualification. Thus, the same attitude serves a social function for volunteer $\mathrm{A}$, and a career function for volunteer B.

The VFI has now been used and tested across a wide range of volunteer areas. When a prototype was analysed against a national survey of American adults the results provided considerable support towards a functional approach of volunteer motivation (Clary, Snyder, \& Stukas, 1996). The VFI also proved reliable when tested in different fields such as with online panellists (Vocino \& Polonsky, 2011) and parks and recreation volunteers (Silverberg, Ellis, Backman, \& Backman, 1999). Across all of the various areas it has been used, the three highest functions have been values, 
understanding and enhancement, though this does vary between different groups (Clary \& Snyder, 1999; Newton et al., 2014). There have also been certain trends noted for the functions, such as the belonging function being negatively correlated against educational achievement (Ferreira, Proença, \& Proença, 2012), volunteers with a high career function finding dissatisfaction in workplaces with less job resources (Lo Presti, 2013), and the career function being negatively correlated with age (Ferreira et al., 2012). Employees who were compelled to volunteer as a part of a corporate social responsibility programme were found to have the same order of VFI functions as other volunteers doing the same activity, but at consistently lower levels (Do Paço et al., 2013). Key criticisms of the VFI include that it is eclectic with little theoretical basis, it does not use an exhaustive list of functions, not all of the functions have the same level of generality, and the responses are subject to desirability bias (Shye, 2010; Wilson, 2012).

\section{Stages of Volunteer Motivation}

There are several different stages of volunteer motivation which will likely change as a volunteer continues with an organisation. Chacón et al. (2007) have proposed a three stage model of volunteer service: the initial phase, greater involvement, and sustained involvement. During the initial phase, satisfaction of initial motivations to volunteer is the best predictor of satisfaction, and therefore, continued service. This is a contention that has been noted across much of the literature (e.g. Clary \& Snyder, 1999; Douglas \& Rollins, 2007; Houle et al., 2005). As the volunteer continues in their role, their level of commitment to the volunteer organisation becomes the fundamental influence over whether they will stay or not. Eventually, in the last phase, role identity becomes paramount, as for a volunteer to sustain long term involvement, they need to assume the role of the volunteer. At this point the role of a volunteer becomes internalised to the point where it is something that one is, and not just something that one does (Finkelstien, 2009). These stages were confirmed by Jiménez, Fuertes, \& Abad (2010) who went further to chart the specific characteristics of volunteers who had remained into the final stage, having completed over eight years of service. Many of the findings were expected, such as organisational commitment, a strong role identity, and satisfaction with friendships within the organisation. But a surprising result was a higher level of emotional exhaustion noted 
by the experienced volunteers. This seems to suggest that volunteers require there to be an important cost to their actions so that they feel that it is worthwhile. A similar finding was also noted by Stukas, Worth, Clary, and Snyder (2009) who found that matching tasks to volunteer motivations made the volunteerism more exciting and interesting, but also more draining and frustrating.

There are a number of other factors that have been identified as important to the retention of volunteers. One of the most important of these is the need for adequate supervision. Volunteers appreciate managers who understand their motivations (Grimm \& Needham, 2012), have a good understanding and experience working with volunteers (Silverberg et al., 1999), and put in place good support structures (Lo Presti, 2013; McCudden, 2000). Other factors which have been identified as affecting the retention of volunteers include social and organisational recognition (Lo Presti, 2013; Mykletun \& Himanen, 2016), good information and communication (Lo Presti, 2013; Warren \& Garthwaite, 2015), a sense of success from their contributions (Fortier, Auger, \& Froment-Prévosto, 2007; Mykletun \& Himanen, 2016), and fun (Fortier et al., 2007).

\section{Training Volunteers}

When using the term training, is should be noted that this term is used instead of the terms education or learning. As Pells (2008a, p.3) notes;

Training is instruction that is directly related to the employment activity of the trainees, and usually given in their place of employment. This differentiates training from education, which is typically less specific to the trainees' work tasks and most often (but not always) undertaken prior to employment. Both training and education are a subset of the more inclusive concept of learning.

Within this thesis I will exclusively be using the term training, in keeping with usage by the volunteer organisations within this research, and in much of the literature on volunteers (e.g. Huang et al., 2014).

In considering the training of volunteers, it is imperative that the needs of both the organisation and the volunteer are considered. The major barriers towards training for organisations are time and money (Nichols et al., 2005; Pells, 2008a). Generally 
organisations will invest in training if the benefits they receive through productivity gains are high enough to offset the costs (both through putting on the training, and loss of productivity). Training can also offer benefits to the organisation such as increased awareness of company values and brand, and risk mitigation (Babbage, 1990).

Training is most likely to be seen as a benefit for a volunteer when it relates directly to their motivations for volunteering (Newton et al., 2014; Pells, 2008b), and can have many benefits for volunteers including personal growth, helping to pull volunteer teams together, and giving them a shared purpose (Kenwright, 2000). But for many volunteers mandatory training can be seen as a barrier, with the main reasons for this including family, time, and work commitments, lack of relevance, and threatening assessment (Attewell, 2009; Pells, 2008a; Warren \& Garthwaite, 2015). This does not mean that training is a deterrent for all volunteers, as in a number of studies intense training programmes have actually attracted volunteers to a programme (Muckaden \& Pandya, 2016), demonstrating organisational commitment to both the training programme and the volunteer's work (Kinnane, Waters, \& Aranda, 2011; Newton et al., 2014), and leading to higher satisfaction rates (Lavenburg \& Bernt, 2012). But volunteers with different motives will not value training events in the same way, as they will have differing needs and expectations of the volunteering activities, and thus the training event (Newton et al., 2014).

The effectiveness of a training programme will depend upon a combination of both trainee attributes, and the effectiveness of the trainers and delivery methods used (Arthur, Bennett, Edens, \& Bell, 2003; Noe, 1986; Salehi, Strawderman, Huang, Ahmed, \& Babski-Reeves, 2009). Wlodkowski (1997) argues that motivation is inseparable from culture within a learning environment. Differences in cultural experience and demographic both affect how volunteers react to training (Ginsberg, 2005; Salehi et al., 2009), and satisfaction with a training programme will influence not just that training event but also any subsequent learning (Noe 1986). There has been little empirical research undertaken into the effectiveness of volunteer training programmes (Bautista, 2015; Huang et al., 2014). In one of the few studies available, Huang et al. (2014) examined four different methods of volunteer training for truck drivers in the United States. Interestingly those who selected face-to-face training had 
the most engagement with the training, but those who undertook web-based training performed the best with assessment.

\section{General Features of Effective Professional Development}

Though there has been little research into the most effective methods of training volunteers (Bautista, 2015; Huang et al., 2014), there is a wealth of literature detailing the aspects that make up good professional development activities in general workplace environments (e.g. Boud \& Hager, 2012; Eraut, 2004; Murphy \& Calway, 2008; Piggot-Irvine, 2006). The assumption that human resources practices used within paid workforces can be directly transferred to volunteer environments is subject to debate (Laczo \& Hanisch, 1999). However, in the absence of research about volunteer training methods, the features of workplace professional development activities are generally used as the benchmark for both employees and volunteers. These features include: the need for flexibility in the teaching programme, collaborative learning, transfer of learning, and organisational support.

A feature of successful professional development activities is the use of collaborative learning, which allows learners to observe, engage and discover new processes of thinking in action, as they encounter different learning approaches from others within the group (Cervero, 2001). This collaborative approach has also been noted as a successful teaching method within volunteerism. Pairing volunteers with a permanent staff member, or experienced volunteer, has proven in many cases to be a useful tool in helping to ground knowledge back into the workplace (Babbage, 1990; Douglas \& Rollins, 2007).

An aspect that has been regarded by many as one of the most crucial elements to help obtain lasting learning outcomes, is the transfer of learning (the ability to transfer what has been learnt at a training event back into a practical work situation) (Cervero, 2001; Eraut, 2004; Murphy \& Calway, 2008). This transfer process from the abstract to the practical is described by Daley (2001) as a part of the actual meaning making process. Without this contextualisation process, people in diverse roles, and encountering complex situations, must rely on the retrieval of prescriptive knowledge which will tend to help little with complex real-world problems. 
Lastly, in order for learning to translate into practice there must be organisational support of the learner. This organisational support can occur (or not occur) on a number of levels. The wider attitude of the organisation towards learning has been found to affect an employee's confidence in the learning process (Boud \& Hager, 2012; Dailey, 1984; Eraut, 2004), and the attitude of the learner's manager is one of the most crucial factors determining whether a learner will be successful in implementing new knowledge (Eraut, 2004; Murphy \& Calway, 2008). On a more practical level, learners must be provided with the practical resources, such as necessary equipment, money, and information, that they need, if they are to effectively transfer learning back into the workplace (Noe, 1986).

\section{Professionalisation of the Volunteer Sector}

Within New Zealand, volunteer organisations take a variety of approaches to the training of their volunteers. Increasingly there is pressure on many volunteer organisations, and particularly those within more formal workplaces with external contracts, to train volunteers using formal qualifications that sit on the New Zealand Qualifications Framework (NZQF) (Pells, 2008a). This is partly due to the desire of volunteers to receive qualifications that are externally recognised, a finding that is described within the responses of the volunteers later in this report. But it is also due to the increasing pressure for volunteer organisations to professionalise their operations and training systems due to external pressures, such as requirements that are necessitated by government funding (Nichols et al., 2005; Pells, 2008a). This parallels similar pressures felt by volunteer organisations in other countries, such as Australia (Ellis et al., 2014) and England (Nichols et al., 2005; Taylor \& Garratt, 2010). The pressure to professionalise within the voluntary sports sector in England, has been caused by both the requirements of external funders (such as the government), and competition from private providers. This has led to feelings within the sector of centralisation, regulation and uniformity (Taylor \& Garratt, 2010).

\section{Tertiary Education Reforms}

The last 30 years have been a time of huge transition within the educational sector in New Zealand. Starting in the late 1980s and early 1990s, and as a direct result of concerns over the state of the New Zealand economy, education became a means to 
transition people into work (Strathdee, 2011; Vaughan, 2005). The governments of that time believed that people in a post-industrial economy required high levels of skills and qualifications (Strathdee, 2012) and so introduced a single qualifications framework (refer to Table 2.1) with a common system for measuring and recording learning (Strathdee, 2011). New Zealand remains today as one of only a handful of countries with a unified or comprehensive qualifications system (Chan, 2016). The result of these far-reaching changes was to shift education systems away from professional accountability and towards systems of government accountability and audit (Vaughan, 2005). It also broke down the previous distinctions between academic and vocational knowledge, with all forms of knowledge seen as being of equal value, and transferability of learning seen as a key aim (Strathdee, 2011).

Another change was the introduction of a market-based bulk-funding system for education, with funding dependent upon the numbers of equivalent full time students that a provider had (Vaughan, 2005). The unforeseen consequence of this shift was a massive increase in private education providers and programmes, and a "bums-onseats" mentality that tended to emphasise the need for student numbers over quality outcomes. This was addressed in the 2000s by the introduction of the Tertiary Education Commission which was tasked with directing tertiary investment into areas of strategic relevance (Strathdee, 2011; Vaughan, 2005). Both of these models have seen tensions arise between the needs of funding structures which are outcomes based, and learners, employers and industries (Chan, 2016). In a survey of tertiary

\section{Table 2.1: Qualification Types on the NZQF}

\begin{tabular}{|c|c|c|c|}
\hline Level & Certificate & Diploma & Degree \\
\hline 10 & & & Doctoral Degree \\
\hline 9 & & & Master's Degree \\
\hline 8 & Postgraduate Certificate & Postgraduate Diploma & Bachelor Honours Degree \\
\hline 7 & Graduate Certificate & Graduate Diploma & Bachelor Honours Degree \\
\hline 6 & Certificate & Diploma & \\
\hline 5 & Certificate & Diploma & \\
\hline 4 & Certificate & & \\
\hline 3 & Certificate & & \\
\hline 2 & Certificate & & \\
\hline 1 & Certificate & & \\
\hline
\end{tabular}

Note: Adapted from http://www.nzqa.govt.nz/studying-in-new-zealand/understand-nz-quals/ 
education staff the Tertiary Education Union found that institutional pressure to increase completion rates (which are a key funding criterion) put pressure on their staff to pass students regardless of whether they had met the criteria or not (Oosterman, Sedgwick, \& Grey, 2017).

The final major change within the New Zealand tertiary sector has been the increased influence of Industry Training Organisations (ITOs). These bodies were established in 1992 as a result of the Industry Training Strategy, and were seen as a way for industries to control the development and management of their industry training programmes (Strathdee, 2011). However, in the years since, the purpose of these ITOs has constantly shifted according to the changing political persuasions of the New Zealand Government (Strathdee, 2012). In 2002, the new Labour Government argued that the previous market-led approach had led to unsatisfactory results in terms of the quality of training provided, and appropriateness of skills produced (Moses \& Strathdee, 2007). Further, it argued that employers were not investing sufficiently in training and development, and so advocated for a more active relationship with ITOs in developing and reforming training within their sectors. Gradually this has led to tension between the Tertiary Education Commission and ITOs, particularly around areas such as outcomes based funding structures (Chan, 2016; Moses \& Strathdee, 2007). Funding for qualifications is administered by TEC based on a provider's qualification completion rates; however, this does not always work well for industries that would prefer smaller or more flexible training which may not lead to a completed qualification. Presently the New Zealand Government spends three billion New Zealand Dollars per year towards the education of 565,000 tertiary students, including 138,000 within industry based training (Ministry of Education, 2014). 


\section{Method}

The purpose of this chapter is to describe the methodology used to undertake this research project. Firstly, the rationale for the use of a qualitative case study approach is given, and the features of this approach are described. The research method is then detailed, with descriptions of the two volunteer organisations, training events, and data collection methods. Following this the data analysis methods will be explained, both for the VFI questionnaire, and the semi-structured interviews. Finally, the trustworthiness of the findings and ethical issues are explored.

\section{A Qualitative Approach}

Whilst there is a growing body of research into the motivations of volunteers, and some exploration into effective training methods for volunteers, there has been little critical research into the interactions between these two phenomena. The lack of prior research in this area meant that an explorative approach was most suitable for this study. Qualitative research seeks to understand and describe people's experiences as opposed to quantitative research which typically seeks to test hypotheses or theories (Johnson \& Christensen, 2012). The use of a qualitative approach permitted an indepth exploration of the feelings and perspectives of the volunteers in the research, and provided a detailed description of a largely unexplored research area.

Those using qualitative research methods tend to select an interpretative paradigm to inform the assumptions they are making about: the nature of reality (ontology), what is known (epistemology), the inclusion of values (axiology), how the research emerges (methodology), and how the research is written (rhetorical) (Creswell, Hanson, Plano Clark, \& Morales, 2007). For this research a constructionist approach was selected. Researchers who utilise this approach "claim that truth is relative and that it is dependent on one's perspective" (Baxter \& Jack, 2009, p. 545). Constructivist research relies heavily upon detailed description of contextual factors, due to the belief that "reality is constructed by individuals interacting with their social worlds" (Merriam, 1998, p. 6). As the construction of knowledge is believed, within this approach, to be heavily influenced by a learner's context, it is important to understand and describe wider contextual factors as a part of the research. A constructionist paradigm fits well with the use of a case study approach due to the rich 
description that is afforded by this research method (Baxter \& Jack, 2009; Creswell et al., 2007).

There was a small quantitative element to the research, in the form of a widely used standardised questionnaire. This was a minor part of the overall research and used as a point of triangulation to confirm the more significant data from the semi-structured interviews. The intent of this instrument was not to alter the exploratory and descriptive nature of the research, and therefore shift the research as a whole away from a qualitative approach.

\section{Case Study Research Design}

"Case studies are differentiated from other types of qualitative research in that they are intensive descriptions and analyses of a single unit or bounded system" (Merriam, 1998, p.19). Grünbaum (2007) describes six major characteristics of case studies: The study objects are always related to people who are studied in their natural environment. They are based on contemporary (and therefore not historical) phenomenon. They take a holistic perspective, and try to identify and understand contextual factors. The studies are usually qualitative, where the objective is descriptive, exploratory, or explanatory. The researcher has no control over crucial events unfolding in the context. There are numerous data sources utilised by the researcher. And rich contextual accounts are produced to describe the phenomenon.

Merriam (2009) argues that the single most defining characteristic of case study research is the limiting of a study to a particular object, or case. This object of study becomes a bounded system, a single entity around which there are boundaries which fence in that which is to be studied. The boundaries around the case can include time and place, time and activity, and definition and context (Baxter \& Jack, 2009). The collective case study approach (also referred to as multiple case study approach) is used to gain greater insight, by utilising more than one unit of study (Johnson \& Christensen, 2012). Within this approach there is only one issue of concern, but multiple studies are utilised to show different perspectives, and further illustrate the issue (Creswell et al., 2007).

The issue of concern for this multiple case study was the relationship between training and the motivation of volunteers. To investigate this issue two cases were utilised, 
which were the two volunteer organisations, and as will be detailed further below, these two cases were further bound by length of service. Thus, the case study was limited to volunteers from two voluntary organisations, who had undertaken the organisation's training programme, but had a maximum of two years of volunteer service with the organisation.

Common criticisms of qualitative case studies involve issues of reliability, validity and generalisability (Merriam, 2009). The use of multiple methods of data collection, and multiple sources can help to alleviate some concerns regarding trustworthiness of the data. To further improve trustworthiness Yin (2009) calls for a chain of evidence that could be used by another researcher to track through each step of the case. Qualitative case studies, however, will never achieve results that can claim to be generalised across a general population. The intent is instead to provide a rich and detailed description of the bounded system that is being studied.

\section{Research Method and Procedures}

The following section details the methods or tools used to collect the data used for this research. Having settled upon a qualitative case study approach for the project, it was necessary to select research methods that complemented this approach.

\section{Participants}

Investigations into the characteristics of volunteers within New Zealand have shown marked contrasts in the age, gender, household income, and stage of life of volunteers across different areas of the volunteer sector (Charbonneau, Brennan, \& Hercus, 2006). Whilst it cannot necessarily be assumed that consistency of such factors will equal consistency of motivation or experience as a volunteer, it did seem prudent to include the perspectives of volunteers from more than one environment. Two volunteer organisations with contrasting volunteer activities were invited to participate in the study. Meetings with both organisations were held, and once they had agreed to participate, copies of the organisational consent form were signed.

Within each organisation a purposive sample of volunteers with less than two years of experience was sought. The decision to restrict the sample size to those with less than two years of experience was made for two reasons. Firstly, a key aspect of the 
research related to the participant's initial motivations to volunteer and how these motivations were influenced by the induction training that they received. Secondly, the initiation training procedures of both organisations generally occurred over the first two years of volunteer service (though ongoing informal training and professional development activities did continue after this period). Restricting the sample size to those with less than two years of experience attempted to mitigate recall bias by ensuring that the answers were coming from those with recent memories of why they were motivated to join, whilst still including the perspectives of volunteers who had been through the whole initiation procedure.

\section{Volunteer Organisation A}

The volunteers from Organisation A were selected from two fire stations in the Wellington region. Volunteers are required to attend a weekly training night, where informal training is conducted, and news is disseminated. Additionally, new recruits are required to complete mandatory formal training before they can participate fully in firefighting activities which may involve a call out to an emergency situation at any point during the week.

Each of the stations was visited during one of the training nights, and the research was briefly explained to the firefighters. Invitations to participate were then offered to those who met the criteria. Four volunteers responded positively and each was subsequently interviewed.

Of the four participants, three from Organisation A were male and one was female, and they had an average age of 25. Two identified themselves as students, and two were in full time employment. All participants were of European descent, with two identifying themselves as New Zealand European/Pakeha and two identifying themselves as British/English. The length of service of the group ranged from one month to three years, which included one volunteer who fell outside of the initial criteria of having less than two years of service. However, due to the relevancy of their responses, and the fact that they were still undertaking induction training, it was decided to include their responses as part of the research. 


\section{Volunteer Organisation B}

Volunteers from Organisation B are adult literacy tutors providing one-to-one or small group tutoring for adults with low literacy and numeracy skills within a suburb in the greater Wellington region. Before taking on any students, prospective tutors are required to complete a block course and follow up assignments towards a nationally recognised qualification.

Of the three participants from Organisation B, two were female and one was male, and they had an average age of 58. Two identified themselves as being retired and one as in full time employment. All participants were of European descent with two identifying themselves as New Zealand European/Pakeha and one identifying as British. The length of service of the group ranged from one month to two years.

Table 3.1: Study Participant Demographics (Both Organisations)

\begin{tabular}{lllll}
\hline Age & Gender & Ethnicity & Employment Status & Length of Service \\
$16-20$ & Male & English & Full Time Employee & 2-3 Years \\
$16-20$ & Male & NZ European & Student & 1 Year \\
$16-20$ & Male & Pakeha & Student & 5 Months \\
$30-39$ & Female & NZ European & Full Time Employee & 2 Months \\
$40-49$ & Female & British & Full Time Employee & 1 Month \\
$65+$ & Female & British & Retired & 1 Month \\
$65+$ & Male & Pakeha & Retired & 2 Years
\end{tabular}

\section{Difficulties Recruiting Participants}

Whilst it was initially hoped to include the perspectives of a greater number of participants, this proved to be more difficult than expected. In contrast to research with permanent employees, where interviews may be undertaken during work time, any interview with a volunteer will usually happen in their spare time. For many, and in particular for those already busy with work, family, their volunteering, and any other pursuits, participating in additional research can be an extra burden that they are, quite rightfully, unwilling to undertake. This is particularly so for new recruits who are already undertaking induction training. Many of the participants noted how they found it difficult to find the time to complete assessments, and so their generosity in finding more time to participate in this research is greatly appreciated. Time is a valuable commodity within the voluntary sector, and it is a consideration that must be factored into the expectations of any research within this area. 


\section{Data Collection}

Researchers utilising the case-study method tend to be pragmatic and utilise multiple methods and data sources (Johnson \& Christensen, 2012). The two main methods of data collection in this thesis were semi-structured interviews and a short questionnaire. Further information was gathered via visits to the volunteer sites, informal conversations with volunteers, and exploration of the organisations' websites.

Shye (2010) notes the need for rigorous research methods when researching the motivations of volunteers, to avoid eclectic results with little theoretical basis. The use of semi-structured interviews is consistent with any qualitative explorative research which seeks to gain in-depth insight into various phenomena (Denzin \& Lincoln, 2008). Merriam (2009) states that interviewing is the best technique to use when conducting intensive studies into a few selected individuals. The VFI Questionnaire is one of the most rigorously tested and reviewed tools to diagnose the motivations underlying volunteerism (Clary, Snyder, Ridge, et al., 1998). This short quantitative questionnaire was given to participants prior to the interview to ground any motivational findings against a previously tested motivational framework, and to serve as a reference point for themes emerging from the interviews.

\section{Setting}

Prior to the interviews I was invited to attend training nights at both fire stations in Organisation A: to get a sense of the training that goes on, and to meet participants from this organisation prior to arranging interviews. This allowed me to observe training activities first-hand, meet and talk with a number of the volunteers, and get a feeling for the culture of both stations. Following these training nights, contact was made by email with those who had expressed interest, to give further information and to arrange an interview time. Organisation B preferred to send information about the research directly to potential participants, and requested that anyone who was interested was to contact me. Prior to the interviews I was able to visit the centre, meet some staff members, and get a snapshot of the volunteers' environment.

Once contacted by the volunteers, I arranged a time and place for the interview based upon what was most convenient to the participant. Interviews were held in a mixture 
of the organisation's base, participant's workplace, and my workplace. Initially it was hoped to conduct a second interview with participants to try and measure changes in motivation over the intervening time period. But due to the wide range of length of service within the sample group, and the depth of data already captured, this was deemed to be unnecessary. Whilst it certainly would have added some extra detail and insight, it was thought not to be worth the extra inconvenience to a group of people for whom time was already an important factor.

\section{Process}

Prior to the interviews, participants were given the participant information sheet, and once they had arrived at the interview they were asked to complete the participant consent form. Following this process they were given a paper copy of the VFI Questionnaire and asked to complete it, which usually took approximately five minutes. I remained in the room whilst this was being completed, as the questionnaire often elicited questions or comments from the participants which I referred back to during the interview if necessary. Once they had completed the questionnaire, participants were led through a semi-structured interview using the questions from a pre-prescribed schedule. The interviews were recorded on two devices, a portable audio recorder, and my mobile phone, to safeguard against loss of data. Additionally, multiple recordings proved to be valuable when checking the transcriptions, as if one recorder was bumped or obscured, the conversation could still be captured via the other device. Upon completion of the interview participants were thanked, asked to contact me at any point should they have questions or think of something else to add, and informed that they would be sent general themes to comment on in due course.

\section{VFI Questionnaire}

The Volunteers Function Inventory (VFI) is a questionnaire devised by Clary, Snyder, Ridge, et al. (1998) to assess the functions potentially served by volunteerism. Utilising a functionalist approach they argue that people can perform the same actions in the service of different psychological functions. Thus, two people could have the same purposes for volunteering but with these purposes serving completely different functions. Clary, Snyder, Ridge, et al. grouped these functions into six categories:

- values - the opportunity to express values related to altruistic concerns 
- understanding - the chance to gain new learning experiences and/or to exercise existing knowledge, skills, and abilities

- social - the ability to socialise and/or engage in activities viewed favourably by others

- $\quad$ career - to allow career related benefits including preparing for a potential role

- protective - involves processes associated with the ego including protecting from feelings of guilt or negative feelings of the self

- enhancement - the other side of the ego including feelings of positive strivings and to obtain satisfactions related to personal growth and self-esteem

In the questionnaire participants are asked to rate 30 possible reasons for volunteering such as "volunteering makes me feel important" and "I feel it is important to help others" on a scale of one to seven (refer to Appendices F and G for a full list of the questions). Each question relates to one of the functions listed above, with a total of five questions per function.

\section{Semi-structured Interviews}

Interviews are one of the most common forms of qualitative data collection, and necessary when we cannot directly observe behaviour, feelings, or how participants observe the world around them (Merriam, 2009). Semi-structured interviews, or the interview guide approach, involve an interviewer using a pre-prescribed interview protocol containing open ended questions about specific areas of interest (Johnson \& Christensen, 2012). This leaves the interviewer free to explore areas of interest which may arise whilst covering the areas needed.

The questions for the interview protocol were devised early on in the research process, and were submitted as part of both the research proposal, and the application for ethics approval. There were two main focus areas for the interview; the participant's motivations to volunteer, and the perceptions that they had of the training events.

Following some introductory demographic questions, the next questions sought to explore the volunteer's initial motivations to volunteer, and whether these had shifted since they had started as a volunteer. Whilst calling for the use of more rigorous testing procedures when exploring volunteer motivations, Shye (2010) notes that the 
use of standardised lists may have a short circuit effect. This short circuit excludes motivations that are salient for certain sets of people, circumstances or volunteering, but not common enough to have been noted on previous lists. This is to some degree verified by a study by Pells (2008b) who observed a motivation unique to those volunteering in the emergency services. The feeling of adrenalin rush that volunteers experienced when doing emergency services work is one that is quite probably unique to that field, and not categorised in any existing lists of volunteer motivations. As participants had already completed the VFI Questionnaire it was decided to keep these questions on motivation quite general so as to leave the research open to uncharted motivations which would not easily fit within the existing VFI functions.

The remaining questions explored the participant's perceptions of the training. These were loosely based upon the Framework for Culturally Responsive Teaching developed by Wlodkowski and Ginsberg (1995). This framework seeks to provide a teaching strategy that helps students relate learning content to their own cultural backgrounds. This approach is significant to this study, as motivations are influenced by emotions, which are in turn strongly learnt through culture. Thus, for teaching to be effective for people across a range of cultures, it requires a holistic approach where the how, what, and why, are unified and meaningful. In this framework four motivational conditions are listed, which the teacher must create or enhance, in order to have a culturally responsive teaching environment. They are:

- establishing inclusion - by creating an atmosphere where the teacher and students feel respected and connected to each other

- developing attitude - by creating a favourable attitude towards learning by personal relevance and choice

- enhancing meaning - creating challenging, thoughtful, learning experiences that include student perspectives and values

- engendering competence - helping students to understand that they are effective in learning something they value

The questions about training were loosely framed by these four conditions as a way to discover how the training event affected their motivations. Whilst it is acknowledged that all of the participants interviewed for this study were of British or NZ European ethnicity this would not be representative of everyone doing the initiation training for 
both organisations (as noted in a few of the interviews). It is also important to note that, despite the similarities in ethnicities, the differences in age, life experience, and aspirations within the group of participants was wide, and contributed to a wide range of worldviews.

Because the participants of this research came from two different volunteer organisations, and had varying levels of service and experience, all of the interviews were unique. Some volunteers came into the interviews with no thoughts about what they wanted to say, and required a lot of follow-up questions to elicit meaningful answers. Other volunteers entered with clear ideas of what they wanted to say, in particular those who had training experiences which they did not think were optimal. It was important during the interviews to remain impartial, whilst still retaining rapport with the participant by empathising with their point of view. I found the need to resist the temptation to seek the sensational, or to mine for areas of conflict. In all of the interviews there were aspects of the volunteer's experience which were "good", "bad", and even non-remarkable, and it was necessary to remain open to all of these possibilities, and not to prioritise one over the other.

\section{Data Analysis}

The two main methods of data collection for this research required different approaches towards analysis. The data from the VFI Questionnaires required a simple quantitative analysis similar to that used by Clary, Snyder, Ridge, et al. (1998). For the larger, and primary, data from the semi-structured interviews, a qualitative approach was taken using the six phase process of thematic analysis developed by Braun and Clarke (2006).

\section{VFI Questionnaires}

Given the small sample size, and because the questionnaire was not the primary source of data for this research, a simple and pragmatic approach was taken towards the analysis of this quantitative data. Following the approach towards individual data that was used in the initial study outlining the VFI (Clary, Snyder, Ridge, et al., 1998), the means and standard deviations for each participant across each of the various factors were calculated. 
The variances in both mean and standard deviations highlighted the differences in response approach between various participants. To complete the questionnaire, participants were asked to rate each of the factor statements between 1 (not at all important/accurate) to 7 (extremely important/accurate). A simple analysis of responses showed that some participants responded generally higher than others across all of the questions, and some responded with a larger range of responses than others. Due to this variance in approach, little heed was paid to how participants responses varied between each other on any data area. Rather, each participant was analysed according to how their responses varied across the six factor areas within their own response data. Given that the point of the questionnaire was to ascertain which motivational factors the participant thought were the most important, and not how strongly these motivational forces were held in comparison to others, this approach seemed fitting.

Mean and standard deviation values were calculated for each participant across all of the six motivational factors and an overall mean calculated. The means for each factor were then rated from highest to lowest to reveal the factors which were most important to the participant. Overall means were calculated both for each participant organisation, and as a whole. This was not to compare the participant organisations against each other on any one factor, for the reasons mentioned earlier, but to find the factors which were rated higher and lower for each of these groups.

\section{Semi-structured Interviews}

Data analysis for the semi-structured interviews utilised the six phase process of thematic analysis developed by Braun and Clarke (2006). Thematic analysis is a widely used method of data analysis but suffers from being rarely acknowledged and poorly demarcated (Boyatzis, 1998). Braun and Clarke (2006) argue that clear and concise guidelines are required to avoid the "anything goes" mentality that is a common critique of qualitative research. The process of data analysis for these interviews was consciously undertaken at a slow and methodical pace, aligning closely with the phases outlined by Braun and Clarke at every step.

\section{Six Phase Process}


As per the Braun and Clarke model, analysis was a recursive process, and not a strictly linear process. Discoveries in data, or new theme generation, often necessitated skips back and forth across all of the six phases.

Phase one of the six phase process is "familiarising yourself with the data". The choice was made to transcribe the interview recordings personally, which proved to be a long, but valuable, method of data familiarisation. With the interviews lasting between 15-45 minutes long, these transcriptions took a number of months to complete which allowed a lengthy period of time for various ideas to settle. Once they had all been transcribed I read through each transcription at least twice to further familiarise myself with what had been said.

Phase two of the process is "generating initial codes" which was completed by noting interesting features across the data and collating these features together. To begin, each interview transcription was printed out, and data that was deemed to be significant was highlighted, and then assigned an initial code. Once this had been completed for each interview, these highlighted sections were extracted electronically and tagged with the initial code. This process involved some rationalisation of codes, with similar codes being combined, such as "stage of life" and "change of life". During this stage, care was taken to retain codes even if they did not appear to be dominant, and to keep data surrounding the extract to ensure that the sections retained their context.

The third stage is "searching for themes", which began by sorting each code into 34 thematic subsets which were then further consolidated into five major categories: initial motivations, ongoing or developed motivations, training, workplace culture, and miscellaneous.

Phase four is "reviewing themes" which involves refining and reviewing the themes at two levels. Firstly, the collated data for each theme were re-read to check if they fitted coherently within that theme. Secondly, the validity of the various individual themes was considered against the entire data set. At this point I returned back to phase one, and re-checked the transcriptions against the second audio recordings that I had made. This allowed me to both double check the transcriptions for accuracy, and to listen to the entire data set against the initial themes that I had developed. Following this process a series of thematic maps were drafted with the themes mapped out visually. 
Phase five "developing and naming themes" involved the development and reworking of the themes set out previously. Eventually this led to a set of themes within three main areas: motivation (initial and developed), training (positive and negative), and intersections between training and motivation. At this point the themes were developed into a one page map which was sent to all of the participants for feedback. This feedback, where appropriate, was integrated back into the data set, but did not necessitate a major review of the thematic analysis.

The sixth and final phase "producing the report" was completed by the production of this thesis.

\section{Trustworthiness}

The three main areas of trustworthiness in qualitative research are descriptive, interpretative, and theoretical (Johnson \& Christensen, 2012). Descriptive validity refers to the factual accuracy of the account. Two recordings were made of each interview to ensure against loss of data, and these were carefully transcribed and checked against each other. Interpretative validity describes how accurately the meanings intended were ascribed by the researcher, with regards to the participant's viewpoints, thoughts, feelings, intentions and experiences. The most common method of checking for interpretative validity is through the use of participant feedback. All participants were sent a map of themes during data analysis for feedback, which yielded positive feedback. Where suggestions were made these were incorporated back into the pool of data, however, no feedback that was received contradicted any of the major themes. The verbatim transcriptions of interviews were low-inference descriptors, which captured the nature of each conversation as truly as possible. Theoretical validity refers to the degree to which data matches a theoretical explanation. The research began with clearly defined constructs, and then used an instrument (case-study) that was recognised as a reliable measure of these constructs. After themes had been identified from the interview data, they were cross-checked in relation to Clary, Snyder, Ridge, et al.'s (1998) constructs and this process was detailed. 


\section{Ethical Issues}

Any research that utilises human participants requires careful ethical consideration. Ethical approval for this research was requested via the Victoria University of Wellington Human Ethics Committee, and this was granted on 28 August 2015. Clark (1997) notes four ethical principles which are important for educational research: justification, informed consent, confidentiality, and deception.

\section{Justification}

As noted earlier in the report, the intersection between volunteer motivations and training remains an area with little research. It is hoped that any research in this area may contribute to training for volunteers that is both meaningful and increases their motivations to continue in their volunteering. Both organisations approached were eager to participate and utilise the findings from the research.

\section{Informed Consent}

Written consent was first sought from the respective organisations, and then from each participant before collecting any data. Participant information forms were distributed to each participant either during initial inquiries, or prior to the interviews, and these were further explained in person, prior to the start of the interview. The information forms clearly explained the scope of the project, what the participant's role would be, their right to withdraw from the project, and how their personal information would be kept safe.

\section{Anonymity and Confidentiality}

An aim of the research was to respect the privacy of all research participants, including through seeking to ensure their anonymity in any research reports. Whilst every care has been taken to achieve this aim, given the small number of volunteer organisations in the Wellington region, and the small number of research participants, this is probably not entirely possible. Certainly, whilst the exact names of Organisation A and Organisation B have not been given, those familiar with the respective sectors may be able to identify the organisations involved. This was clearly disclosed on the organisational information sheet, and explained prior to the organisational consent forms being signed. Likewise, the small number of research 
participants means that those within each respective organisation may be able to identify some of the participants from their responses. To mitigate this, specific personal information has been removed, and when quotes were deemed to be controversial they have not been assigned to participants. All personal data has been kept on password controlled computers or in a locked filing cabinet, and participant data coded before distribution (e.g. to the research supervisor) as part of the research process.

\section{Deception}

Every care has been taken to remain open and transparent to everyone involved with the project regarding the aims and hopes for this research. I have tried to remain open minded to the data received, and to avoid pre-conceptions of what I wished to find. All of the participants will be offered a summary of the findings following the completion of the research, and both organisations have requested presentations of my findings. 


\section{Results of the VFI Questionnaire}

This section briefly summarises the results of the VFI questionnaire. These results are first discussed as a whole and then compared between the two volunteer organisations and various demographic groupings.

In the questionnaire each respondent was asked to rate thirty reasons for volunteering as to how important or accurate they are in relation to their reasons for volunteering. Each reason is rated from one to seven, with one being "not at all important/accurate" and seven being "extremely accurate". Each of the 30 values relates to one of the volunteering functions devised by Clary, Snyder, Ridge, et al. (1998), with each function being represented by five questions (for a full list of the questions, refer to Appendices $\mathrm{F}$ and $\mathrm{G})$. The volunteering functions are:

- values - the opportunity to express values related to altruistic concerns

- understanding - the chance to gain new learning experiences and/or to exercise existing knowledge, skills, and abilities

- social - the ability to socialise and/or engage in activities viewed favourably by others

- career - to allow career related benefits including preparing for a potential role

- protective - involves processes associated with the ego including protecting from feelings of guilt or negative feelings of the self

- enhancement - the other side of the ego including feelings of positive strivings and to obtain satisfactions related to personal growth and self-esteem

Firstly it must be acknowledged that given the small sample size, any attempt to provide substantial quantitative analysis of the data would be meaningless. However, the data that was captured does provide an opportunity to make general comparisons between the seven volunteers, and the two organisations, as well to provide a reference point for the answers from the semi-structured interviews.

At an individual level it is clear to see that some volunteers responded with generally higher answers, and/or with less variance than other volunteers. VL23 $(M=1.7)$ for example had a low average response across all of the factors, whereas VL24 $(M=5.1)$ had a much higher average response. Similarly, whilst VL26's answers were remarkably consistent, with a standard deviation of less than 1 in each of the six 
factors, others such as VF19 and VL24 had large variances in standard deviation, indicating that they were more inconsistent in their answers within each of the functions. Due to these variances in response, it was impossible to make straight comparisons of individual answers across the various functions. Rather, each participant or group was analysed according to how their responses differed across the six factor areas within their own response data.

Table 4.1: VFI Results Organisation A

\begin{tabular}{lccccccccc}
\hline & \multicolumn{2}{c}{ VF25 } & \multicolumn{2}{c}{ VF19 } & \multicolumn{2}{c}{ VF22 } & \multicolumn{2}{c}{ VF21 } & All \\
& $M$ & $S D$ & $M$ & $S D$ & $M$ & $S D$ & $M$ & $S D$ & $M$ \\
Career & 3.2 & 2.0 & 5.8 & 1.3 & $\mathbf{5 . 6}$ & 0.5 & 1.0 & 0.0 & 3.9 \\
Enhancement & 6.0 & 0.9 & 4.0 & 1.4 & 4.4 & 0.5 & 1.2 & 0.4 & 3.9 \\
Protective & 3.4 & 1.6 & 3.6 & 1.1 & 4.0 & 0.7 & 1.2 & 0.4 & 3.1 \\
Social & 5.6 & 0.5 & 3.2 & 1.6 & 3.4 & 1.1 & 2.0 & 1.4 & 3.6 \\
Understanding & 6.4 & 0.5 & $\mathbf{6 . 2}$ & 0.8 & $\mathbf{5 . 6}$ & 0.9 & 1.8 & 1.1 & 5.0 \\
Values & $\mathbf{7 . 0}$ & 0.0 & 5.8 & 0.4 & 4.8 & 1.3 & $\mathbf{4 . 2}$ & 2.6 & $\mathbf{5 . 5}$ \\
\cline { 2 - 9 } (all functions) & 5.3 & & 4.8 & & 4.6 & & 1.9 & & 4.1
\end{tabular}

Note: The highest factor scores have been noted in bold and the lowest in italics.

Table 4.2: VFI Results Organisation B

\begin{tabular}{llllllll}
\hline & \multicolumn{2}{c}{ VL24 } & \multicolumn{2}{c}{ VL23 } & \multicolumn{2}{c}{ VL26 } & All \\
& $M$ & $S D$ & $M$ & $S D$ & $M$ & $S D$ & $M$ \\
Career & 6.0 & 0.7 & 1.0 & 0.0 & 2.0 & 0.0 & 3.0 \\
Enhancement & 4.2 & 1.1 & 1.6 & 1.3 & 4.4 & 0.9 & 3.4 \\
Protective & 2.8 & 1.5 & 2.0 & 1.4 & 2.4 & 0.9 & 2.4 \\
Social & 3.8 & 1.8 & 1.0 & 0.0 & 2.4 & 0.9 & 2.4 \\
Understanding & $\mathbf{7 . 0}$ & 0.0 & 1.8 & 1.1 & $\mathbf{6 . 0}$ & 0.0 & 4.9 \\
Values & $\mathbf{7 . 0}$ & 0.0 & $\mathbf{2 . 8}$ & 2.5 & $\mathbf{6 . 0}$ & 0.0 & $\mathbf{5 . 3}$ \\
$M$ (all functions) & 5.1 & & 1.7 & & 3.9 & & 3.6
\end{tabular}

\section{General Results}

Across all of the volunteers the highest average response was for the values function $(M=5.4)$, followed closely by the understanding function $(M=5.0)$. The lowest two functions were social $(M=3.1)$ and protective $(M=2.8)$. Interestingly these rankings stayed remarkably consistent between the two organisations with both having values highest, understanding second, and protective and social as their bottom two functions. This mirrors previous research which has shown values to be the highest 
function, along with understanding and enhancement (Clary \& Snyder, 1999; Newton et al., 2014). The function that varied the most between volunteers was the career function, with some volunteers rating this very highly, and others ranking it as not important at all. The reasons for this will be discussed further below.

Table 4.3: VFI Results Combined Organisations

\begin{tabular}{lcccccc}
\hline & \multicolumn{2}{c}{ All } & \multicolumn{2}{c}{ Org A } & \multicolumn{2}{c}{$\begin{array}{c}\text { Org B } \\
(n=3)\end{array}$} \\
& $M$ & Ranking & $M$ & Ranking & $M$ & Ranking \\
Career & 3.5 & 4 & 3.9 & 3 & 3.0 & 4 \\
Enhancement & 3.7 & 3 & 3.9 & 3 & 3.4 & 3 \\
Protective & 2.8 & 6 & 3.1 & 6 & 2.4 & 5 \\
Social & 3.1 & 5 & 3.6 & 5 & 2.4 & 5 \\
Understanding & 5.0 & 2 & 5.0 & 2 & 4.9 & 2 \\
Values & $\mathbf{5 . 4}$ & 1 & $\mathbf{5 . 5}$ & 1 & $\mathbf{5 . 3}$ & 1 \\
$M$ (all functions) & 3.9 & & 4.1 & & 3.6 &
\end{tabular}

\section{Results by Demographics}

Variances in response were explored against demographic factors of age, employment status, ethnicity, and gender. When examining responses in each of these groupings, it is noticeable that some groupings allocated higher or lower scores in every function than the average participant (for example, note the lower than average responses in every function by those who are retired, or over 65 years of age, and higher than average responses by those aged 16-20). For this reason, it is once again necessary to examine results as to how they compare based on their relative ranking within the same demographic grouping, as opposed to rating each function across the various demographics groupings.

\section{Employment Status}

In terms of employment status, it is unsurprising to note that those who were students ranked career as their second highest rated function $(M=5.7)$, which is far above where it sits on average as the fourth most highly rated function. As noted earlier, values is generally the most highly rated function, however, this only appeared as the third most highly rated function for students $(M=5.3)$ who noted understanding $(M=$ $5.9)$ as their highest rated function. By contrast those who were retired rated career $(M$ $=1.5)$ as their lowest rated function, with social $(M=1.7)$ just marginally above it. 
Table 4.4: VFI Results by Employment Status

\begin{tabular}{lcccccccc}
\hline & \multicolumn{2}{c}{$\begin{array}{c}\text { Employed } \\
(n=3)\end{array}$} & \multicolumn{2}{c}{$\begin{array}{c}\text { Student } \\
(n=2)\end{array}$} & \multicolumn{2}{c}{$\begin{array}{c}\text { Retired } \\
(n=2)\end{array}$} & \multicolumn{2}{c}{$\begin{array}{c}\text { All } \\
(N=7)\end{array}$} \\
& $M$ & Ranking & $M$ & Ranking & $M$ & Ranking & $M$ & Ranking \\
Career & 3.4 & 5 & 5.7 & 2 & 1.5 & 6 & 3.5 & 4 \\
Enhancement & 3.8 & 3 & 4.2 & 4 & 3.0 & 3 & 3.7 & 3 \\
Protective & 2.5 & 6 & 3.8 & 5 & 2.2 & 4 & 2.8 & 6 \\
Social & 3.8 & 3 & 3.3 & 6 & 1.7 & 5 & 3.1 & 5 \\
Understanding & 5.1 & 2 & $\mathbf{5 . 9}$ & 1 & 3.9 & 2 & 5.0 & 2 \\
Values & $\mathbf{6 . 1}$ & 1 & 5.3 & 3 & $\mathbf{4 . 4}$ & 1 & $\mathbf{5 . 4}$ & 1 \\
$M$ (all functions) & 4.1 & & 4.7 & & 2.8 & & 3.9 &
\end{tabular}

\section{Age}

When examining age profiles it should be acknowledged that, due to both the small sample size, and demographic profiles, the results for those aged 65 and over is identical to the results for those who were retired, with career and social both ranking lowly in relative importance. Results in the other two age ranges stay fairly similar to the rankings overall, other than a slight difference in the importance attributed to enhancement by those aged 35-50. Whilst this is ranked as the third highest ranked function overall, it is only the fifth ranked amongst this demographic.

Table 4.5: VFI Results by Age

\begin{tabular}{lcccccccc}
\hline & \multicolumn{2}{c}{$\mathbf{1 6 - 2 0}$} & \multicolumn{2}{c}{$\mathbf{3 5 - 5 0}$} & \multicolumn{2}{c}{$\mathbf{6 5 +}$} & \multicolumn{2}{c}{ All } \\
& $M$ & Ranking & $M$ & \multicolumn{2}{c}{$(n=2)$} & \multicolumn{2}{c}{$(n=2)$} & \multicolumn{2}{c}{$(N=7)$} \\
Career & 4.9 & 3 & 3.5 & 3 & 1.5 & 6 & 3.5 & 4 \\
Enhancement & 4.8 & 4 & 2.7 & 5 & 3.0 & 3 & 3.7 & 3 \\
Protective & 3.7 & 6 & 2.0 & 6 & 2.2 & 4 & 2.8 & 6 \\
Social & 4.1 & 5 & 2.9 & 4 & 1.7 & 5 & 3.1 & 5 \\
Understanding & $\mathbf{6 . 1}$ & 1 & 4.4 & 2 & 3.9 & 2 & 5.0 & 2 \\
Values & 5.9 & 2 & $\mathbf{5 . 6}$ & 1 & $\mathbf{4 . 4}$ & 1 & $\mathbf{5 . 4}$ & 1 \\
$M$ (all functions) & 4.9 & & 3.5 & & 2.8 & & 3.9 &
\end{tabular}

\section{Ethnicity}

For those of British ethnicity the values function is rated most highly $(M=4.7)$, and by a significant margin ahead of the understanding function $(M=3.3)$ which, though rated second most highly, is actually closer in rating to social $(M=2.9)$ and enhancement $(M=2.9)$. For this grouping the career function is rated quite 
convincingly as the least important of the functions $(M=1.7)$ which may be influenced by the lack of students in this demographic. The New Zealand European grouping is fairly similar in results to the overall rankings.

Table 4.6: VFI Results by Ethnicity

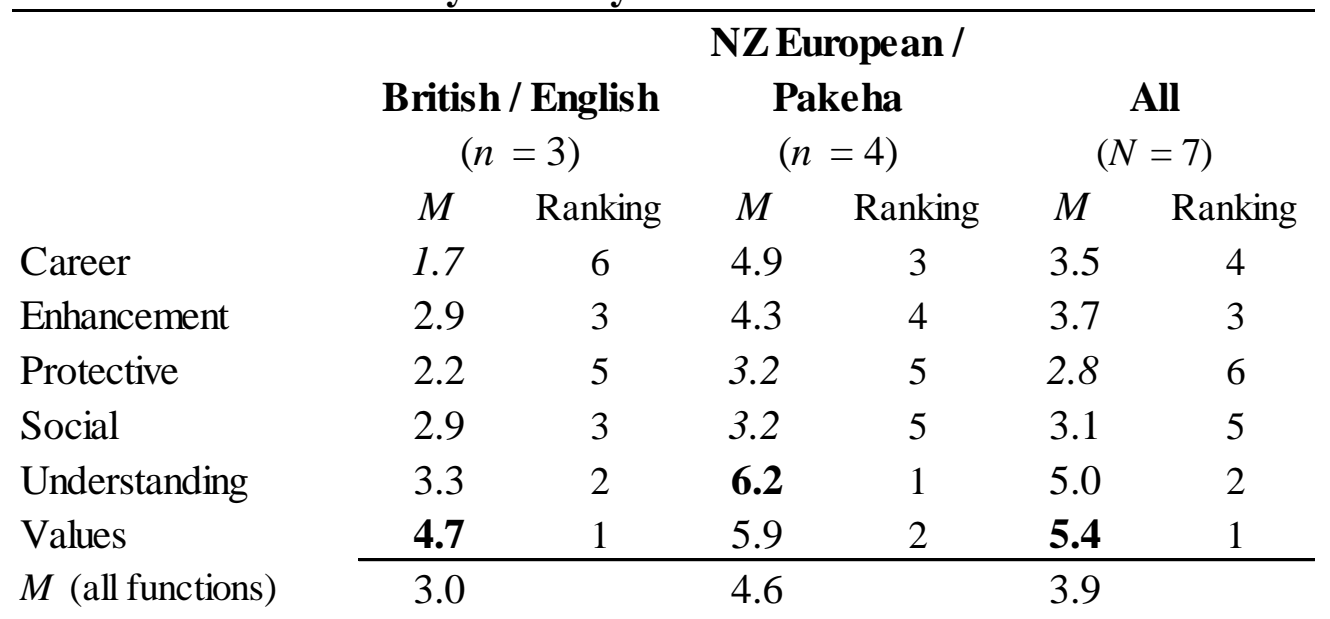

\section{Gender}

Lastly, when exploring gender differences, males stay relatively similar to the overall average, however, with understanding rating as the most important function $(M=6.1)$, slightly ahead of values $(M=5.9)$. It is noticeable, however, that for females the values function is rated emphatically as the most important $(M=4.7)$. This is again followed by understanding $(M=3.5)$, with the other four rating fairly similarly to each other, though significantly lower than the first two functions.

Table 4.7: VFI Results by Gender

\begin{tabular}{lcccccc}
\hline & \multicolumn{2}{c}{ Male } & \multicolumn{2}{c}{$\begin{array}{c}\text { Female } \\
(n=3)\end{array}$} & \multicolumn{2}{c}{$\begin{array}{c}\text { All } \\
(N=7)\end{array}$} \\
& $M$ & Ranking & $M$ & Ranking & $M$ & Ranking \\
Career & 4.2 & 4 & 2.7 & 3 & 3.5 & 4 \\
Enhancement & 4.7 & 3 & 2.3 & 4 & 3.7 & 3 \\
Protective & 3.4 & 6 & 2.0 & 6 & 2.8 & 6 \\
Social & 3.7 & 5 & 2.3 & 4 & 3.1 & 5 \\
Understanding & $\mathbf{6 . 1}$ & 1 & 3.5 & 2 & 5.0 & 2 \\
Values & 5.9 & 2 & $\mathbf{4 . 7}$ & 1 & $\mathbf{5 . 4}$ & 1 \\
$M$ (all functions) & 4.6 & & 2.9 & & 3.9 &
\end{tabular}


Overall it is noticeable that values and understanding are consistently ranked as the two most highly ranked functions, across almost all of the demographic areas. By contrast, the protection function ranks consistently low in the order of functions, and does not appear in the top three ranked functions of any demographic grouping. The most fluid of the functions is the career function, which is particularly influenced by age and employment status. 


\section{Participant Perspectives from the Semi-Structured Interviews}

In this section the perspectives of the participants, as described within the semistructured interviews, are summarised and explored. The first part of the chapter details the motivations of the participants to volunteer for each of the organisations. As the interviews progressed it became clear that there were two categories of motivational factors that were being described by the volunteers. Firstly, the initial motivations which impelled the volunteer to enrol with the organisation, and then secondly, the motivations that had developed, or been enhanced, since enrolling with the organisation. These developed motivations were sometimes a result of the training, or at other times a result of other factors, such as camaraderie, or inspirational experience. Both the initial and developed motivational factors are then analysed against the results of the VFI Questionnaire detailed in the previous chapter.

The second part of this chapter examines the volunteers' perceptions of the training events that they were asked to complete. These responses are described according to the positive and negative reactions that volunteers had within each organisation.

\section{The Motivations to Volunteer with Organisation A}

The first organisation invited to participate in the research (Organisation A), is a branch of firefighters based in Wellington New Zealand. Participants were selected from two different stations operating out of separate suburbs within the Wellington region.

All volunteers at Organisation A are required to attend a weekly training night where the squad is updated on relevant news, and training occurs. This training can vary between watching safety videos, undertaking physical drills such as testing hoses, or conducting simulations of a firefighting scenario. In addition to this regular ongoing training, new volunteers are required to complete a number of mandatory assessments which fulfil unit standards on the New Zealand Qualifications Framework (NZQF). These assessments are paper-based and cover fundamental skills such as "operate aerial appliances in emergency situations". Until these initial unit standards are completed, volunteers are restricted in what they are able to do (e.g. attend fires, 
operate pumps). Camaraderie is seen as a key ingredient in the success of these stations, and new recruits are partnered with experienced volunteers during training exercises to "show them the ropes". Many volunteers will turn up long before the training begins to work on equipment and get ready for the evening, and remain behind after the training for drinks, and to socialise with each other.

\section{Initial Motivations}

The four volunteers from Organisation A noted a number of reasons for volunteering, but the most significant initial motivational factors were altruism, career goals, personal challenge, and the influence of others.

\section{Altruism}

The most common motivational factor to be given by the volunteer firefighters in Organisation A was altruism. As discussed in the Literature Review, altruism is the desire to help others without any expectation of personal reward. This was expressed in a number of different ways by the volunteers such as: "I quite like to help people" (VF22), "I actually like giving back" (VF21), and "helping out those most in need" (VF25). It was never the only motivation expressed, and a number of the volunteers acknowledged that being able to help people was a convenient benefit in the fulfilment of another, more self-orientated, motivation.

I: $\quad$ But in terms of your service here, it's very much motivated by wanting to get skills ...

VF19: Yeah, and at the same time it's a good way of doing it. By serving the community for free.

For each of the volunteers there was a complicated mixture of an altruistic desire to serve their communities, and self-orientated desires to benefit themselves. This included improving themselves physically (e.g. to get fitter), mentally (e.g. to learn new skills), socially (e.g. to progress career goals), or psychologically (e.g. to feel better about themselves).

There is a danger within self-reported research that people will respond with answers which they believe to be socially desirable, such as providing altruistic reasons as motivations to volunteer at the expense of more self-orientated reasons (Aydinli et al., 2016; Shye, 2010). This was confirmed to me informally by an experienced firefighter at a training night: 
They always put on the [application] form that they want to make the world a better place, but when they first come in most of them really just want to drive around in the trucks with the lights going.

\section{Career Goals}

For the two youngest volunteers, who were both students in New Zealand secondary schools at the time, career goals were an important factor. One of these volunteers specifically chose the firefighters as preparation for a future career in farming or the forestry industry.

I: $\quad$ So why did you choose to do it here, as opposed to [another emergency services branch]?

VF22: Because I was looking at going into something, in the, like a forestry, or a farming career, so I thought this one would be most sort of um, suitable to that.

The second of these volunteers (VF19) joined the firefighters with the wish to utilise the experience and qualifications to enrol in the New Zealand Army. For this reason the accrual of new skills and knowledge was a highly motivating factor, as was the chance to receive a formal qualification.

\section{Personal Challenge}

Two firefighters noted the aspect of personal challenge as a motivation to volunteer. For the first volunteer (VF25) this primarily related to physical fitness, but at times involved psychological challenge as well. The psychological challenge related to times of stress which had required the volunteer to push through a task which was daunting.

For the second volunteer this related to a change in life, which allowed the opportunity for a new challenge.

VF21: Um, so basically I um, my kids have left home, or in their like, sort of their final years of leaving home, so I've got a bit more time on my hands and firefighting has always been sort of something in the background for me, for actually looking into ... And it was possibly a bit of a challenge I suppose, bearing in my mind [my gender], my age, and everything. But um, and I also look at it as I, I like staying fit and healthy too and this type of role would definitely, sort of need that. 
Having children leave the home is a significant life event for parents, and this volunteer has used this milestone event as impetus for a new challenge.

\section{The Influence of Others}

The last motivational factor which was evident for volunteers in Organisation A was the influence of others. This was expressed in two forms, as a legacy influence (e.g. the memory of a childhood role model), and as a present day energiser. The legacy influence was noted by three of the volunteers. VF22 described fond memories as a child hanging around the fire station with family members who were volunteers. VF19 similarly remembered back to family friends living down the street who volunteered in the fire service. The most significant example, however, was given by VF19 who detailed the early influence of a family friend.

VF21: As a child growing up my best friend's dad, who I spent a lot of time with, he was a firefighter in the village that we lived in, and yeah, so he was a bit of, he was my inspiration to be quite honest out there. And I always looked up to him, he was someone sort of, yeah, that was always, yeah, he was a huge part of my life, so to me, It was like - why not? You know, I can do this.

In all three of these examples there existed a significant role model, or role models, that the volunteers wished to emulate in their volunteer service.

Three volunteers noted specific people who they either consulted, or who encouraged them to volunteer, prior to applying. VF21 had workplace colleagues who volunteered as firefighters and they provided encouragement to apply. VF19 found the fire service by way of a civil defence course which was completed at a police station. The last volunteer to articulate personal encouragement was VF25, who was encouraged to join by a work experience scheme at school.

VF25: Well I found out about this place through ... a work experience placement ... and they set me up with these voluntary firefighting services. And because they know ... what kind of jobs you want to get into ... they got me into this voluntary firefighting, and I've been here ever since.

In at least two of these examples the encouragement was given to somebody who was already actively exploring volunteer options (whether as a firefighter or elsewhere), so it is unlikely that this encouragement in isolation would have been enough to compel them to join Organisation A. External encouragement, however, does appear to be an 
important energising factor which may motivate potential volunteers, who already have existing motivational factors, to act on them and sign up as a volunteer.

\section{Developed Motivations}

As well as capturing the motivations that encouraged the participants to initially apply to be a volunteer, the interviews captured motivational factors that developed after beginning with the organisation. Some of these developed as a result of the training and others developed from non-training related factors. For the volunteers in Organisation A the most significant factors were camaraderie, peer relationships, and new skills.

\section{Camaraderie}

Many of the volunteers identified the camaraderie within the station as an important motivation to stay. This was expressed in a number of ways by the volunteers including friendship, fun, and acceptance. It was stressed by VF25 that this developed motivation of new friendships was in addition to initial motivations, and had not replaced them.

VF25: Well the old motivations I had have kept exactly the same throughout the years I've had. And the extra added motivation I have, is I've got a great crew with me, and they're all those people that I consider as great friends, and I really enjoy the company of every single person there.

A sense of fun and good humour was seen by VF21 to be an important factor, and one that draws a wide group of people together. This social aspect is fostered by the likes of people staying behind for drinks after the weekly training sessions.

VF21: There's a wide range of age groups, so they know when to pull everybody in, it's actually really good. There's no harm in having a bit of humour and banter in everything as well, which they very much encourage - they encourage the social sort of side as in to get to know each other as well better I think. So yeah, no, I think it's, it comes across as a real friendly team.

An emphasis on the social side of the station contributes to new volunteers feeling readily accepted into the group. VF19 noted that this collegiality is more important for the volunteers than having a fancy setting, or the latest equipment. Ultimately, and probably due to the heavy reliance on teamwork within firefighting, the friendliness 
and acceptance shown by the existing volunteers will play a huge part in determining whether a volunteer in this environment will choose to stay on as a volunteer.

VF19: Like you can have a flash as station and have a bunch of boring people and you wouldn't enjoy it. But this place, it's not the flashest place, but the people basically they're all a good bunch of people to work with, and they're not um, they're all real accepting, like if you notice new people come, they're all pretty friendly and that, and they have a bit of banter as well, it's not all, it's not all serious faces and that, so I enjoy it.

As VF22 put it: "I look forward to coming here. So it's not one of those things that sort of, you wake up in the morning and you think 'oh no, I've got to come and do this"'.

\section{Peer Relationships}

Contributing to the strong sense of camaraderie within the organisation is an effective use of semi-formal coaching relationships between experienced volunteers and new recruits. These are semi-formal in the sense that they are generally allocated by the station chief or another senior firefighter at the start of the informal training nights, but not formally assigned or agreed to. A number of volunteers noted examples where these relationships had continued further. Two volunteers gave examples where they were offered extra assistance, outside of the normal training times, by experienced volunteers.

VF21: ...we only really have one training session a week. And I understand the constraints about that but, for somebody who's onboarding sometimes you can sort of give a bit more time and they've been accommodating there. They've actually said to me, "look, yeah if you want to come in on the weekend, as well" ... a few of them are sort of around and they're happy to come in.

In addition to helping out with skills and knowledge related to the new volunteer role, VF19 noted that many of the other volunteers shared tips on preparing for a future career outside of the firefighters.

VF19 Yeah, all the guys are real good about doing that. Like, "oh, you'll be doing this in the Army, so it's good that you understand this". And even um, furthering some of the stuff that we do. Like, when I'll be talking about something, and some of the guys at the end of the night will go have a drink and that, they go and explain something else. They're like "oh you could do this as well", and he says "when you're in the Army you'd have to do it this way" and stuff like that. 
These strong relationships not only help the new recruits to settle in quickly, and feel accepted as a part of the team, they also developed a feeling of reciprocity. As the volunteers feel accepted and able to contribute, they develop a desire to give back and contribute as much as they can to the team.

VF21: ...they've lost a few experienced firefighters so it's about getting on board to actually be able to support them, cause they are quite sort of low on numbers. More the experienced level. There's no way I can get to that level anyway in this time for this year, but hopefully from now, if I can help them out, I think it's just making it easier on the whole station really as well as having another set of hands that can do the role.

The usage of semi-formal peer learning relationships between experienced volunteers and new recruits in Organisation A has contributed to a healthy team culture that stretches further than the standard training requirements of the weekly training nights. This has not only helped the new recruits to feel accepted, and accustomed to their roles, it has helped to contribute to a sense of organisational commitment for the volunteers.

\section{New Skills}

The final developed motivation that was noted by the new volunteers, was the acquisition of skills and knowledge. The chance to learn new skills, and understand new concepts, was noted by two of the volunteers as a motivation that they had developed since joining Organisation A.

VF21: I love to learn new things, and so that's part of it for me, I'm learning something very new, and all these names [laughs] for all the pieces of different equipment and things, [laughs] yeah it's like "woah, who came up with that name, what's that" you know?

A key factor of this knowledge acquisition for VF22 is that it is not restricted to firefighting and can be applied to other contexts. Given that a primary motivation for VF22 to volunteer was to gain new skills and experience towards a future career, this is an important factor.

VF22: ... you learn, sort of, things that are useful and other things, not just firefighting and... it's just valuable stuff to know for later on in life ... Like, I guess the teamwork sort of thing. Working as a team, and knowing about the different equipment and, stuff like that. 
For VF22 the motivation to gain new skills and knowledge is heavily related to this volunteer's initial motivations to volunteer which were discussed earlier. For VF21, however, this was not identified as an initial motivation to volunteer, and arose as a result of the learning that occurred whilst training.

\section{The Motivations to Volunteer with Organisation B}

The second organisation invited to participate (Organisation B), is an adult literacy education provider based in the Wellington area. This organisation is one branch of a parent body which provide adult literacy education throughout New Zealand.

Volunteers in Organisation B are required to completely mandatory training before they are allowed to begin tutoring students. This training programme has recently been changed and now involves completing a National Certificate on the NZQF. New recruits must attend a training block of five days, delivered by the parent body, and then successfully complete a series of competency based assessments in order to receive this qualification. Prior to the new system, volunteers were required to complete a different qualification developed by the parent body which was not registered on the NZQF. Following the block course participants are assigned an experienced tutor as a support person to guide them through the assessments, which include 20 hours of contact time tutoring students. Upon successful completion of the qualification they are assigned permanent volunteer roles tutoring adult literacy students either individually, or occasionally, in small learning groups. Once they have completed the qualification there is little formal interaction between the tutors, other than via professional development activities which occur six times per year on topics such as first aid, and accessing online catalogues.

\section{Initial Motivations}

The three volunteers from Organisation B detailed a number of motivational factors which were important to their decision to volunteer, the most significant of which are detailed below. These factors are altruism, utilisation of existing knowledge and skills, and career enhancement. Due to the small response rate for the research it is acknowledged that there are a number of voices missing that may have provided additional reasons for volunteering. Some of these silent voices are discussed via the 
second-hand observations of one of the volunteers who has an additional paid role within the organisation.

\section{Altruism}

Similar to the firefighters in Organisation A, the adult literacy tutors rated altruism as one of the most important reasons to volunteer. This was described in a variety of ways such as "to try and help people" (VL26), and "doing something to help people" (VL24). For VL24 it was making a contribution to the wider community.

VL24: I just wanted to feel like I was, like a part of my community, I was contributing, I was doing something to help people who are maybe not fitting in as easily as they could.

VL23 was the only volunteer not to explicitly list some form of altruism as an initial reason to volunteer. Interestingly though, this volunteer went on to detail subsequent altruistic motivational factors which are detailed below, and developed as a result of the training.

\section{Utilisation of Existing Knowledge and Skills}

Another prominent motivational factor was the utilisation of previously learnt skills and knowledge. As the average age of volunteers from Organisation B was higher than those from Organisation A, it is not surprising that these volunteers had life experience and learning which they wished to pass on. For all three of the volunteers this acquired knowledge had come through a mixture of qualifications and prior teaching experience in related fields.

VL24: I have a teaching background, and I have quite a bit of experience teaching, lots of kids, but I've done some volunteering with adults and teaching adults as well ... education's what I love and I guess I feel really fortunate, I feel really blessed actually that I'd never struggled with reading ... so if I've got that confidence and those skills, then being able to share that with people and help other people seems like a really amazing thing to be able to do.

For the two volunteers who were retired, this desire to utilise their knowledge and skills was amplified by the spare time now made available to them.

VL26: ...I just thought I've got these qualifications, I've got spare time now that I'm retired, so why not use them and see if I can make a difference. 
For VL26 it appears that this newly available free time allowed for the utilisation of knowledge and skills in a way that was not formerly possible due to previous restrictions on time. However, in the case of VL23, this appears to be more significant. Prior to retirement this volunteer had been working with learners in another workplace, but through a mixture of retirement and changes in the learning needs of the other organisation, found increasingly less opportunities to interact with these learners.

VL23: I've been working casually ... before I retired and after I retired ... and it really perks me up going talking to [learners in a previous workplace]. ... But um, I thought I really missed it ... I'm always much jollier after I've been teaching people.

The "perkiness" that this volunteer gained from the learning interactions came as a result of being a difference for the learner, and feeling that their knowledge and expertise were needed, and being utilised. Thus, within the VFI framework of motivational functions it would sit best within a protective (protecting from negative feeling of the self) or enhancement (to obtain satisfactions related to self-esteem) function, as well as with understanding (the chance to exercise existing knowledge, skills, and abilities).

\section{Career Related}

One of the adult literacy volunteers, VL24, was in a unique position, as both a parttime employee of the organisation, and a volunteer tutor. Thus, a key motivation to volunteer was to gain further insight into the requirements of the voluntary role.

VL24: My role, it sometimes feels like there's a part that I can't fully understand for tutors, because I'm not doing it myself, and as [part of my role] I think I should be able to do what they do ... so I just think it makes sense that I have the qualification that they have and that I go through the same process that the tutors go through and being trained, so I can properly understand it and help them.

Thus, for this volunteer, the desire to volunteer is driven by a motivation to gain skills and experience in order to be more effective in their employment role. This was the only example, within the group of people interviewed from Organisation B, of a career motivation. 


\section{Silent Voices}

As noted above, VL24 has a role working for Organisation B, and therefore, is in a unique position to observe many of the applicants who have applied and been accepted for volunteer roles. After the standard volunteer interview had been completed, the opportunity was taken to pose further questions to VL24 seeking firsthand observations of volunteers who had applied.

I: $\quad$ Just off the top of your head ... what are the general motivations that tend to come out, both in the interviews and on the forms?

VL24: Well I guess, um, it's split between people who, they'll, volunteer, but they want the qualification. Because they, it, it's a really good qualification to have for employment, you know, it's nationally recognised and that's what they want...

I: $\quad$...so why do you think they want the qualification?

VL24: To get paid work.

These answers emphasise a strong career motivation which has not been entirely captured in the responses from Organisation B given the small sample size. The volunteers are given training towards a nationally recognised certificate in the teaching of literacy and numeracy, which is required for employment in most foundational level education and training positions. It is a qualification that is transferrable to a variety of other employment opportunities, and so many of the volunteers who apply are primarily motivated to gain this qualification, as well as the teaching experience that goes with it. Note that all of the volunteers are expected to complete a set length of service once they have completed the training (usually two years depending on how frequently they volunteer) so this motivation is not necessarily a deterrent for the organisation to accept those with this motivation as volunteers.

The second set of motivations that VL24 described was more altruistically and socially motivated.

VL24: So people who, actually they just want to volunteer. Some people are lonely ... we've got people who, they just want to give back, they want to volunteer. They um, it's something they’ve been thinking about for a while and, and you know, this is the right time for them to do it. But we have existing volunteers who are motivated by, you know, wanting to socialise, 
or you know, um ... do something with their time. Maybe they don't work, or they're retired.

You know we get quite a few older people who have got more time on their hands so, yeah.

The altruistic motivation has already been discussed in detail, however, the motivation to alleviate loneliness was only briefly alluded to in one of the volunteer's answers. This does, however, appear to be a valid reason for many people wanting to volunteer with Organisation B, particularly for those who have either retired or are out of work for various reasons. For some, it may be that volunteerism lets them utilise their previously developed skills and abilities so that they do not feel that these skills have lost their usefulness (and possibly that they are also losing their usefulness).

This motivation of filling up spare time (albeit with a productive and worthwhile activity) is not a particularly desirable response for the volunteer to give, a point that was acknowledged by VL24.

I: $\quad$ Do you think the motivations that people put on the form, and the motivations that people put in their interview are generally, um true? Or do you find that actually often people put something on the form but actually you can tell there's something else going on?

VL24: Well yeah, I think, most of the time they're, they're as truthful as they're willing to be but I guess the loneliness part, you would never write on the form, "I'm actually pretty lonely and I think this will be a good way to meet people and socialise".

This suggests, similar to the informal observations of the experienced volunteer in Organisation A, that volunteers may only disclose motivations that they believe to be socially desirable. This could occur at a conscious level, a sub-conscious level, or both. Volunteers may be worried about the social stigma that would arise if people found out that they were volunteering to alleviate loneliness, or to drive in a big truck with the lights on, and disclose instead a motivation that they believe people will more readily accept. Or, it may be that volunteers do not want to admit to themselves that they are feeling lonely and looking for a way to alleviate this, and so convince themselves that they are volunteering in order to make the world a better place.

\section{Developed Motivations}

Volunteers from Organisation B also discussed motivational factors that had developed as result of the training, and other factors. The most significant of these factors were inspirational insight and intellectual challenge. 


\section{Inspirational Insight}

All of the volunteers noted experiences where they had encountered stories or experiences which had convinced them of the value of the volunteer work that they were a part of. For VL24 this came via a role within the organisation, where first-hand experiences were shared by tutors of the progress that was being made with students. Similarly, VL23 was inspired by the stories that were shared during the training event and the insight that this gave into the struggles of people with low literacy. This developed a new sense of altruistic concern that had previously not been evident when applying to be a volunteer.

VL23: During the training they really gave us an insight into the amount of stigma attached to not being able to read and write, and the personal suffering that goes on as a result. And the other day there was a man standing at the, um, spices section of Countdown, and I was going back and forth getting the tea and so on, and I went to get a spice and he said to me "do you know, if they have cinnamon here?", and there was lots of cinnamon there, and I realised, he probably, his wife had asked him to get cinnamon and he just couldn't read the labels. He was standing there for ages you see, before I went up. So I don't think I would have thought of that before. So I have got an insight into the fact that this is really a blight on their life that does have a real sense of shame, and something they try and hide, and stays with them. And it would be really nice to, but it wasn't what was motivating me to do it, but in hindsight, I, I see that um, if you can help them turn that corner and have less of a horror in their life it would be quite nice.

VL26 was the volunteer with the most experience of the three, and reflected upon the advances (and setbacks) that had been made with those receiving tutoring. For this volunteer, contributing to the progress being made by these students was a clear motivation to continue as a volunteer.

\section{Intellectual Challenge}

The desire to utilise existing skills and knowledge has already been noted as an initial motivation to volunteer, but for two of the volunteers, the prospect of encountering new intellectual challenges, and developing their skills and knowledge further, was a new and added motivation for them. For VL23 this challenge related to applying existing skills and knowledge to a new set of learners with significantly lower literacy levels than previously encountered. 
VL23: They get very low level literacy learners sometimes, and the challenge of ... someone who's really quite, you know, can't recognise letters or words as an adult, um, and it is a, a bit more of a challenge, and that's something that I haven't really dealt with at an adult level. So I'm quite interested to see how I can apply what I know to, such a low level of literacy, yeah.

As noted earlier, VL26 has the longest length of volunteer service with Organisation $\mathrm{B}$, and this has included tutoring students with a wide range of intellectual and developmental challenges.

VL26: Look I find it's intellectually challenging, because I can um, like the guy with um, foetal alcohol spectrum disorder, I think I've ended up with learning and knowing more about that than anybody else in the organisation, just because, you know you want to know why things are happening and what's come about. You get these people with other complaints, with the autism, and alzheimers, and why they've got learning difficulties, and you know I just find that's challenging trying to, find out what they are.

This has led this volunteer to conduct independent research into a number of these conditions, and try a variety of different approaches to achieve better results for the students. The challenge of working out what is going on for the students, and the best approach to use, is a developed motivation that has been developed as a result of volunteer experience.

\section{Analysis of Volunteer Motivations Using VFI Results}

The findings of the semi-structured interviews generally relate closely to the results of the VFI Questionnaire. They are briefly compared here, starting with the functions identified by the VFI Questionnaire, and then considering the aspects of the interviews that are not easily allocated. The purpose of this comparison is to ensure that the findings of the interviews are consistent with the results of a rigorously tested theoretical approach in order to enhance the trustworthiness of the findings.

\section{Values}

The values function describes motivational factors that provide the opportunity to express values related to altruistic concerns. This function is commonly found to be the highest rated factor and was rated (on average) the most highly by the volunteers who completed by the questionnaire. This was also reflected in the responses given in the interviews, with all of the volunteers noting that motivations such as "giving 
back" and "helping people" were important for them (though for one of the volunteers this was an aspect that was developed later). The developed motivation of "inspirational insight" also relates to this function, as the insights inspired volunteers with a passion to help others.

\section{Understanding}

The understanding function describes motivational factors that provide the chance to gain new learning experiences and exercise existing knowledge, skills, and abilities. It was rated the second highest function, and was ranked consistently highly across both organisations, and all of the individual responses. It was the most highly rated function for students, and those aged 16-20. This relates strongly to a number of motivations expressed by the volunteers, including the motivational factors of personal challenge, utilising existing skills and knowledge, developing new skills, and intellectual challenge. It is not surprising to see that the younger volunteers rated this function highly, as they were predominantly volunteering in preparation for future careers, and thus new learning experiences would be valuable to help prepare them for this. It is, however, a little surprising that it does not rank as the most important function for those who are retired, and aged above 65, as the utilisation of existing skills and knowledge, and intellectual challenge, were such strong themes for these volunteers.

\section{Enhancement}

The third most highly rated function on the questionnaire was enhancement, which describes motivational functions that involve feelings of positive strivings, and those used to obtain satisfactions related to personal growth and self-esteem. This function relates strongly to the initial motivation of personal challenge, and may have been linked by some volunteers to inspirational insight and social interaction (as described within that section). It is perhaps surprising to see this ranked so highly on average as it is not particularly ranked by any of the volunteers (with the possible exception of VF25), or in any of the demographic areas, and does not feature strongly in the interview responses. It is possible that this is an aspect that people are less likely to discuss openly, or that the interview questions were not framed to capture sufficiently. 


\section{Career}

Three of the volunteers expressed a strong career motivation, whereas for others this was not an influence at all, which made this the most variable of the six motivational factors. For three of our volunteers this was the lowest ranked function, whilst one ranked it as the most important, and another as the second highest. The career function describes motivational factors that allow career related benefits, including preparing for a potential role, and this was rated on average as the fourth highest function by our volunteers. It was ranked as the second most important function for students, but the lowest rated for those who were retired. Clearly this is a very important factor for some volunteers, but not for others, and is closely linked to age and employment status. This was reflected in the interviews with the three volunteers who ranked this function in their top three, all noting this as a motivational factor in the interviews.

\section{Social}

Perhaps surprisingly, the second lowest function as rated on the questionnaire was the social function, which describes motivational factors that provide the ability to socialise or engage in activities viewed favourably by others. This would seemingly relate to a number of motivations identified by the interviews such as the camaraderie factor, as well as the influence of others (role-model figures), and strong peer relationships. It is possible that due to these being factors that generally developed over the course of their service, as opposed to initial motivations, these were omitted by the volunteers when completing the questionnaire. Nevertheless, it is surprising that this was not rated more highly given the responses in the interviews.

\section{Protective}

Lastly, the protective function describes motivational factors which involve processes associated with the ego, including protecting from feelings of guilt or negative feelings of the self. This was rated as the least important function overall by the volunteers, but it was ranked more highly by those who were retired, and as the second highest by VL23. This corresponds with the responses from VL23 indicating that tutoring helps to "perk me up", as well as the responses from both of the retired volunteers that they wished to ensure that their acquired skills and knowledge were 
still being utilised. It is perhaps one of the motivations that may suffer the most from the self-reported nature of both the questionnaire and interviews, with volunteers, as noted earlier, not being willing to share a motivation, such as to avoid loneliness, or loss of usefulness.

\section{Other Motivations and Summary}

There were a few motivations described by the volunteers in the interviews which did not clearly relate to a single VFI function, or alternatively, related to different functions for different people. For example, emulating the good deeds of an important childhood role model could relate to an enhancement function (feelings of positive strivings), a values function (the opportunity to express altruistic concerns), or a social function (the ability to engage in activities viewed favourably by others).

Nevertheless, overall it can be seen that the findings of the semi-structured interviews correspond fairly strongly to the results of the VFI Questionnaire. The values and understanding functions related highly in both, and the protective function was lowly rated by all but a couple of volunteers. In both the interviews and questionnaires the career function was ranked variably, dependant largely on employment status and age, with these variabilities reflected similarly in both data retrieval methods.

\section{Volunteers' Perceptions of the Training Programme for Organisation A}

The volunteers at Organisation A have two forms of training, the preparation and assessment of a series of formal and nationally recognised mandatory assessments, and the informal practical training that occurs at weekly training nights. The formal assessment component comprises a number of unit standards (modular assessment blocks) on the New Zealand Qualifications Framework, and volunteers are restricted in their activities until they complete specified unit standards. So, for example, Unit Standard 3285 "demonstrate knowledge of protection of personal safety at vegetation fires" must be completed before the firefighters are allowed to attend a live fire in a rural setting. The informal practical training includes drills such as rolling out and checking hoses, fire simulations, and learning to use equipment properly. The volunteers' perceptions of both of these training aspects were sought, and these 
responses are summarised in terms of those aspects of the training that were positive, and those that were negative.

\section{Positive Aspects of the Training for Organisation A}

There were a number of aspects of the training that the volunteers in Organisation A found to be helpful. They enjoyed the mixture of formal and informal training, learning that they determined to be relevant and transferable, and the chance to interact and learn from experienced peers.

\section{Mix of Formal and Informal}

The volunteer firefighters in Organisation A appreciated the mix of formal and informal training, which captured both practical skills and theoretical knowledge.

VF25: Both [types of training] have equal values. You can't be a good firefighter if you're not fit enough and don't have the practical, hands on skills, to perform the task. And you can't be a good firefighter if you don't know what a branch is, or what, what a fourth, what the difference between a rubber, and a percolating and a non-percolating hose are. You can't be a good firefighter without the knowledge and without the practical skills. Yeah, so they're both equal.

They noted that without the more formal training structures, the informal training could become a bit overwhelming, as they were constantly confronted with equipment, procedures, and techniques which they did not fully understand. However, the practical training was invaluable to reinforce and contextualise the knowledge that they had learnt in the formal training.

\section{Interactions with Experienced Peers}

A feature of the informal training that was appreciated by most of the volunteers was the opportunity to learn from a range of experienced volunteers. This peer learning was generally effective, and helped to develop relationships between the new recruits and the rest of the squad.

VF21: [The initial training has] been focussed at aiming me to get through, and um, so I've covered things--- it's more sort of someone coaching me, which has been great, and I don't generally have the same person each week that I go along to training, which is good as well because they've got different ways of thinking and how they cover things, but they're generally seniors that I am sort of coached by ... You get to know the squad better, um, because when you're 
under circumstances that can be quite intense people react differently and if you know them a bit better it's actually easier.

As noted earlier, these peer learning relationships sometimes led to the new recruits and experienced volunteers meeting outside of the regular training hours for extra assistance. Two of the volunteers emphasised the openness of the experienced volunteers to help to accommodate them to their roles.

VF19: Like, there's no, no one stopping you from coming down here. ...the other fella who just walked in before ... we come down and we just pull out a truck and we train on it, just to get the training, and that's good. When you go back on a Wednesday night, because you've already done training on there, and they go "oh have you already done this" and you go "yep, I've had some experience on it" and that's good because then they can critique you on where you, where you're going wrong and that.

Clearly these relationships between volunteers must be fuelled by, and in turn help to grow further, the collegiality within the station. Thus the peer learning relationships, and the collegiality within the station, help to reinforce and grow each other.

\section{Relevant Learning Content}

All of the firefighters found at least some part of the training to be useful, and most found it to be invaluable. Typical of their answers was this response from VF22: "Like um, it was good stuff, it wasn't like that sort of useless stuff you get taught sometimes that's of no real point. This stuff is all sort of stuff you'll actually need to know." Given the dangerous nature of firefighting, safety was a paramount issue, and the volunteers stressed the importance of completing training to ensure that they kept themselves (and others) safe when fighting fires. The priority given to safety training was reassuring to one volunteer, who took comfort in the fact that all of the volunteers had completed similar safety protocols.

VF21: I think it was, it actually covers how serious they take it to me, and that we are all safe. Like I say when you're dealing with things like fire, it's um, yeah. And it's knowing that we've all been taught the same things. You can't just literally let anybody in to, just cover this, because you've got to cover everything yourself and have everybody's back around you, you're supporting each other.

For another volunteer, the transferability of skills to other contexts was important. 
VF19: I mean, you're learning new skills about firefighting which is what I want to do, and at the same time there, also, it's basic, like, basic life skills, like problem solving and that. A lot of the stuff we do is problem solving, because obviously going to emergency situations you've got to figure it out, which is really good to use. Especially when you go back into other situations.

It is evident that the relevance of the learning content was a positive factor for the volunteers. This was especially noted in relation to the transferability of the skills learnt, and importance of the learning content to keep them safe in their service.

\section{Negative Aspects of the Training for Organisation A}

Other aspects of the training were found to be unhelpful by the volunteers. They felt that they had little control over the learning content, and there were difficulties with how the training was structured and delivered.

\section{Issues with Learning Content}

The firefighters felt that they had very little control over either the content or delivery of the formal training components. They all described the formal qualifications as being heavily prescribed, with no flexibility in learning content. When this related to safety protocols, this gave comfort to some of the volunteers (as noted earlier), however, for other volunteers this led to a compliance mentality towards the learning.

VF22: A lot of [the formal training] is just sort of I have to do it because I have to do it. So it's... 'cause I don't really enjoy, and some of the stuff in the books are sort of, I find it's a bit over the top some of the safety stuff and that, but I just do it because everybody has to do it.

This rigidity of content is possibly due to a number of factors including the importance of safety protocols within this context, the fact that the training is externally recognised on the national framework, or even the hierarchical nature of Organisation A. The outcome is that volunteers feel that they have no say in the learning process, and this had an adverse effect for some, on their perceptions of the training.

The lack of flexibility caused other issues for the volunteers, with some feeling pushed hard, and others not pushed enough. Three of the volunteers noted that they felt stretched by a number of the training aspects. Two found it challenging to fit the training around responsibilities at work, home, and with friends, whilst another 
struggled at times with the physical nature of the training due to an existing medical condition. One volunteer, however, noted that the informal training was not challenging enough physically, due to the high range of ages and abilities within the group.

VF19: Sometimes we'll go, go to an area, have like, pretend that there's a fire up there. And so, because everyone's at different, different fitness levels and that, they can look a lot more tired than others, but for some of us who are pretty fit and that, you get up the hill, you go up once and you come back down, and they go "oh that was an awesome job, really easy", and it's like "it's only been ten minutes". [laughs]

The inability to customise training to individual needs can lead to some feeling stretched, whilst leaving others feeling unchallenged. Personal challenge ranked highly amongst the volunteers as a motivational factor, so this could clearly have an adverse impact for those with this motivation.

\section{Issues with the Structure and Delivery of the Training}

Additional to the questions surrounding the learning content, there were a number of criticisms regarding the delivery of the training. This included both the formal and informal aspects of the training, and mainly centred on deficiencies of structures and systems to deliver the training efficiently.

When discussing the formal elements of the training, some volunteers expressed disappointment with the pace that they were progressing at, and the communication of how the formal training was to be completed. This was of particularly concern to those with a high career motivation, who were highly motivated to complete formal assessments.

VF19: I've only done one [unit standard], so it's not really--- 'cause they're quite slow on that paper work kind of stuff.

I: Yeah, would you like to have done more?

VF19: Yep. Ideally I would have liked to have been qualified last year. And they, because it's for unit standards, unit standard took one night, literally ... so, [a head office representative] was going to set up a course, where you got all three unit standards in a weekend, that's how easy it is to get. But it just hasn't, it kept falling through. So it is frustrating that it's taking so long.

Despite there being widespread appreciation for the peer learning system during the informal training nights, a lack of structure hindered this aspect for some volunteers. 
One volunteer noted the lack of training for those providing this peer support, as well as the lack of prior planning as to who would provide it. This sometimes led to inconsistent training and a repetition of learning content.

VF19: There was no structure, when I first got here. So the new guys, it would be ideal to have the new people, one person teaching them, and they go through like, like modular through the different things, rather than--- because what I found was we'd get to here, and they'd be like "who's going to take the new recruits?", they chuck you here, you go here, you go here, and you wouldn't have people knowing where you're up to. So you'd have to, they'd almost be reteaching you every single week the same things you've already done.

For many of the volunteers a more transparent training programme, or process for training, would have helped to increase their confidence in what they were being taught.

\section{Volunteers' Perceptions of the Training Programme for Organisation B}

The adult literacy tutors from Organisation B are required to attend a formal training workshop before they are allowed to engage in any tutoring for the organisation. Following this workshop a range of assessments need to be completed towards a formal qualification. These assessments include some practical assessment work, as well as a number of written tasks. Prior to 2016 the tutors completed a learning programme (the Certificate in Adult Literacy Tutoring) created and run by the national body, which did not sit on the New Zealand Qualifications Framework (NZQF). In 2016 however, the requirements were changed and all new tutors were required to complete The National Certificate in Adult Literacy and Numeracy Education (NCALNE) which is a level five National Certificate on the NZQF.

\section{Positive Aspects of the Training for Organisation B}

There were a number of aspects of the training that volunteers from Organisation B found to be helpful. They identified that the training had provided a range of useful content, a good learning atmosphere, and helpful ongoing tutor support.

\section{Relevant Learning Material}


Two of the three volunteers identified a variety of helpful learning content within the course. Valuable content areas included practical ideas for teaching adult learners, the different ways that people learn, and addressing the needs of Māori learners. For both volunteers, and despite the initial reservations of one of them, the course proved to be a necessary preparation for the demands of teaching adult literacy.

VL23: And looking back on it, of course it's hindsight, but it would have been a mistake to just dash in there. I thought I could just dash in there and do it, but when they were talking about the low level of people who were coming, they weren't just say, students who wanted a bit of extra help, or someone who was at work and just needed help writing reports, some of them who would come would be very reluctant to come, and very hesitant, and have to have a lot of courage to show up at the door and all that kind of thing.

Despite initial concerns about completing the assessments for the course, this volunteer found that the learning from completing the assessments was valuable, and the learning and discovery involved in completing assessments was enjoyable.

\section{Enjoyable Atmosphere}

All of the volunteers agreed that the atmosphere within the workshops was friendly and enjoyable. This provided a relaxed and comfortable environment to learn.

VL24: Yeah, I think there was a nice atmosphere. Yeah, I think so. I think everyone seemed to feel quite comfortable, straight off. ... I think it seemed, everyone seemed really happy, and people took part in all the activities.

The use of group activities and collaborative learning activities encouraged the volunteers to participate and enabled input from a range of voices.

\section{Ongoing Tutoring Support}

Following on from the course there was a support system for the volunteers which assisted them to navigate their way through the assessments, and integrate the new ideas into practice. This involved an experienced tutor meeting up with the new recruits following the training, and being available to talk through any comments or concerns. For one of the volunteers, this was particularly helpful, and they relied on this support person, as well as other experienced tutors within the organisation, for support following the training. 
VL24: So we've got a tutor support person, who's a senior tutor here. ... then after [the training] the tutor support person met, made two different times that all the trainees could go to, so we could go through exactly what we needed to do for the assessment. And then, I've talked with another tutor who's, she already tutors here, she's got another qualification, but we've been talking about how it's going, and like setting each other, like, deadlines to submit and stuff. ... it's been quite comforting ... knowing that you can contact someone if you've got questions.

For new tutors with anxieties around assessment, as well as practice, this additional support can be very beneficial.

\section{Negative Aspects of the Training for Organisation B}

Unfortunately there were a number of aspects of the training that the volunteers found to be unhelpful and at times demotivating. A number of issues with the learning content were identified, as well as issues with the structure and delivery of this content. All of the participants from this organisation came into the training with extensive experience and qualifications but these were not recognised. Additionally, one volunteer described the externally mandated requirements as an unhelpful influence on the training.

\section{Prior Knowledge and Qualifications Not Recognised}

The three volunteers from Organisation B had significant experience and advanced qualifications (i.e. higher than the level five qualification that was required), in closely related fields such as education and linguistics. It was a source of frustration for these volunteers, that this previous knowledge and experience was not recognised in any way.

VL26: I know in [another voluntary organisation] they looked at, um, RCC - Recognition of Current Competency, and RPL - Recognition of Prior Learning. This organisation seems to be unaware of it. Um, which is nonsense really. Because I, I would suggest that my, my knowledge in some of these areas is a good deal greater than what's in these bloody unit standards.

VL23 was surprised at being required to do the training despite prior learning: "I was hoping to be able to do the volunteer work with the experience and knowledge I've got ... you know, the training was kind of, thrust upon me". Though most (but not all) of the volunteers eventually acknowledged that the training was of some value, there were still many content areas which did not elicit new learning. 


\section{Issues with Learning Content}

Because of their prior knowledge, many of the volunteers came into the training with expectations of what would be covered, and were disappointed with gaps in the learning content. For example, VL23 noted the absence of any discussion on linguistics.

VL23: The other thing that was challenging was the lack of discussion about language itself. Which I feel in a training course for adult literacy, there should be a real grasp of what language is and how it works, in order, and how the brain functions with language, in order to teach it properly. But there was no mention of that at all.

One of the volunteers took this criticism further, and ultimately concluded that there was little learning content of any real value for potential tutors. This volunteer believed that the primary focus of the course was to fulfil externally mandated assessment requirements, and that there was little to no focus on aspects that would actually help the tutors to be good adult literacy tutors.

VL26: [sigh] It wasn't particularly motivating. In fact, my concern, and it's probably a bit unfair, but, but my concern really was standing back and looking at it and thinking there are people, wellmeaning people, who want to come along and volunteer and try and help some of these people with literacy and numeracy issues, and this stuff isn't helping them at all, cause there was, there was nothing in it that taught them about how to teach. It was about filling in forms just to meet the unit standard requirements. And that's nothing. I mean it's rubbish.

The most contentious aspects of the training for the participants related to Te Tiriti o Waitangi, and to Māori learners. None of the volunteers interviewed were of Māori heritage, but one of the volunteers had studied much of this content in previous qualifications, and so felt that the content was mostly a repetition of prior learning. One volunteer who had immigrated to New Zealand found this aspect to be unexpectedly useful to her, but also found it challenging when forced to speak the language. The remaining volunteer found this material to be particularly confronting, and divisive for non-Māori. This volunteer told the story of a volunteer leaving the training programme as a result of this content.

VL: I took my car, and took another guy who was actually volunteering at the CAB [Citizen's Advice Bureau] across to the first session, which was a Saturday session, and [laughs] I went to pick him up for the second session, and he came out and said he just couldn't hack it, because the first session had all been about Māori issues in education, and, well ... they were 
coming on pretty strong, and, and there were, we were getting a paper right at the end of the day, and I can't remember who it was, but it was one of the Māori radicals, and basically blaming us Pakeha for everything, and um, and this, this guy was a Scotsman, and it just completely turned him off to the whole programme. It was just, it was over the top really. I mean there was no, there was no middle ground, no discussion, it was just, everything was slating the Pakeha. And, which is not necessarily unjustified, but um, it was um, it wasn't helpful.

Discussion of Te Tiriti o Waitangi is an important part of education within New Zealand society, and particularly so for adult literacy tutors, as Māori learners make up a high percentage of those struggling with adult literacy issues (Literacy Aotearoa, n.d.). Unfortunately, some volunteers felt uncomfortable and out of place with the way in which this content was approached.

It is also important to note that this was not the only example provided of volunteers dropping out as a result of the training. Two of the three volunteers had heard of a number of people who had pulled out during the training.

VL24: We had a couple of people who dropped out after the first day. So I guess, they were the people, but they didn't tend to, they weren't saying much anyway? So I guess...

The two volunteers did not personally know the other people who had pulled out, and so could not answer any further as to why they did not return. It is fairly safe to assume, however, that due to the timing of the withdrawals, most were somehow related to either the content or delivery of the training.

\section{Issues with the Structure and Delivery of the Training}

As with Organisation A, the volunteers in Organisation B felt that they had little choice in relation to the learning content and the assessment of the training programme. One suggested that this actually ran against the very principle of "learner centred learning" that was being taught at the course.

VL: I felt, well um, the thing about the training was, it was talking about learner centred learning, but I felt it was, I know she was trying to be learner centred, but the actual training itself wasn't learner centred. Because it was all set out what we, so it wasn't really a mirror of what we were, she had us doing little activities but it's not quite the same thing. 
Further problems were noted with the delivery of the course, with a volunteer feeling that the tutor did not respect input that was offered. This left this volunteer feeling disempowered, and hesitant to offer further input to the group.

VL: ... she had "gap fill exercises" which she was calling "cloze exercises". And a cloze is where you leave out every nth word, like every fifth or every seventh, and it's an assessment tool. And you just don't care what word you do, that's a "cloze test". But she was calling all the "gap fills", "cloze", and I felt that since we were professionals etcetera, we should be using the right term. But she was quite scathing, she said “oh, well you're just being, that's just semantics”. And so I didn't feel she respected my input, or she wasn't willing to learn from the ... Yeah, she was very dismissive of that, and she kept calling them "cloze test", and I thought "those are gap fill". [laughs]

It is noteworthy that for those volunteers training in 2016, the course was being run for the first time in a new format. Perhaps because of this, there were a number of aspects of the training that were either unclear or ill-defined.

VL24: Because ... it was the first time it's been run as the base training for the organisation, so uh, the tutor kept saying that we were the guinea pigs ... So it's been challenging to understand what they want from us in terms of the assessment, because I think they've been figuring that out for themselves. So, yeah.

Learners ultimately want to have confidence in both the training that they are undertaking and the people who are providing it. Being called "guinea pigs" undermined the learners' confidence in whether the course was adequately equipped to deliver the training optimally. The volunteer who had completed the previous training programme (the Certificate in Adult Literacy Tutoring) similarly noted problems with both the assessments, and the organisation of the course in general.

VL26: The CALT had four, four assessments from memory, that had to get sent off, and they're sent off in a bundle, and they said "oh, be about 12 weeks before you hear". I said "pardon?" I thought, you know, as a learning organisation you don't wait for 12 weeks to tell people they've either succeeded or failed from something. I thought it was bad so, actually turned out it was closer to 14 or 15 weeks before I got the results back.

The lack of assessment detail was particularly notable to those who had done previous study, and led to a sense of confusion about what was expected and how things were to be marked. This was not helped by a reportedly inaccurate description of the 
assessment process, which led one of the volunteers to rush to complete assessments that were not actually due for months.

VL24: After the first two days the trainer said, she gave us this print out with what she said we should do in the two weeks between the next block course. And basically I looked at the list, and it looked like I had to complete all the assessments by the next block course, so I think I spent the next week, like I was doing stuff, like either working or looking after my children or doing those assessments, from like six in the morning till ten at night because I was trying to get it all done. But then, when we went back, she said "oh no, you don't have to have it all done" it was like [groans] "oh, but you gave me that list!"

This volunteer found the extra workload required to fulfil the assessment requirements was difficult to fit around work and family requirements. Garnering the motivation to complete these assessments was made more difficult when it was believed that the content was irrelevant, and the only purpose was to fulfil the requirements of the course. VL26 noted that at times this belief led to a "tick the box" attitude, where many volunteers simply fulfil the requirements of the course, with little real engagement with the content.

VL26: I'm always open for new learning. So, that was, that was never an issue. It was, just turned out the quality of it I thought was, not good. And I heard other people say other, other tutors at [Organisation B] ... "well you know you just, you just play the game. Fill in the forms, play the game".

This feeling of irrelevance was a major de-motivator for the training, and led to disengagement with the learning process.

\section{Driven by External Forces}

As noted earlier, VL26 described many of the aspects of the course as irrelevant, and believed that they were included to appease the requirements of the National Qualification, and for government funding, rather than to help the volunteers to be more effective as tutors.

I: $\quad$ So what does NCALNE get you, in terms of what you can do as opposed to the other one?

VL26: Nothing, it's just the level five National Certificate. It's more recognised.

I: $\quad$ Sure. So why did you elect to that as...?

VL26: Oh I thought there might be some more learning involved. Um. Oh, and the fact that TEC want all the tutors who are doing teaching programmes that are funded by them to have NCALNE. So, it's a minimum thing. 
NCALNE, the government funded national qualification, is now mandated for all incoming literacy tutors to the organisation, and in the future may be extended as a requirement for all existing tutors regardless of their previous experience or qualifications. VL24, speaking from a role within the organisation, expressed concern that this may see many of the (mostly older) existing volunteers leave Organisation B.

I: $\quad$ So have you had any of those people who've just, the training has just been the deal-breaker for them?

VL24: I think we're going to. Because it's, because it's now, this, the NCALNE block, that training is now the basic requirement for any tutors, at any [branch], I think we're going to have that as the deal-breaker. So in future if, if more pressure comes, which I think a lot of it, it's all from the Tertiary Education Commission ... So I think in the next year or so, there'll be more pressure for all tutors to have it. And then we're going to start losing our older tutors I think, who don't have it, who don't want to study.

I: Oh, who haven't done it in the past, and will now need to do it?

VL24: Yeah. They've got a minimum qualification which was what [the head office] asked for before, which was the Certificate in Adult Literacy Tutoring, but they don't want to do anything else, and they're not going to commit to it, and I think that's what's going to happen.

On the one hand the advantages of studying towards a nationally recognised qualification are substantial, both in terms of government funding, and attracting volunteers with a career motivation to the organisation. The inflexibility of this qualification, however, appears to have led to reduced learning outcomes for at least some volunteer tutors.

\section{Summary}

The semi-structured interviews outlined a range of motivational factors that were thought to be significant. Volunteers within both organisations identified altruistic factors as being important, and there were also volunteers from each organisation who expressed a career related motivation. A sense of challenge (whether physical, intellectual, or psychological) was another motivation shared by both organisations though they were expressed in different ways by the different groups of volunteers, probably due to the differing nature of the volunteer service. The generally older volunteers from within Organisation B, were keen to utilise their existing knowledge and skills, and received positive feelings from these learning interactions. By contrast, many of the volunteers from Organisation A were motivated by the development of 
new skills and learning. Interactions with experienced peers was a positive ongoing motivation for both groups, with the stories of peers from Organisation B inspiring the volunteers, and the active mentoring of those within Organisation A motivating them. These findings from the interviews were generally consistent with the findings of the VFI Questionnaire.

In terms of the training, there were also remarkable similarities in the answers given by both groups, despite the differences in volunteer profiles and training style. Volunteers from both organisations appreciated when the learning content was relevant, and enjoyed the support of experienced volunteers. In addition to these factors, the mixture of formal and informal training was a successful part of the training for Organisation A, as was the enjoyable training atmosphere of Organisation B. Both organisations had issues with the learning content, and structure and delivery of the training, which discouraged their volunteers. Common complaints included little learning choice, inflexibility of the training, a lack of learning structures, and gaps in learning content. Additionally, volunteers from Organisation B noted the lack of recognition for prior knowledge and experience as a demotivating factor. 


\section{Discussion}

The purpose of this research was firstly to investigate motivations for volunteering, and then to further explore whether these motivations were affected by the training that these volunteers were required to undertake. This is summarised by the main research question: How are the primary motivations of volunteers affected by the initial training that they are required to complete? Whilst there is a growing body of research into the motivations of volunteers, and a limited body of research examining the effective training of volunteers, both of which were discussed in the literature review, there has been little research that tries to examine the relationship between these two factors. This relationship between the motivation of volunteers and the training that they undertake is important for organisations to understand, as most formal volunteer environments provide some form of initiation training to volunteers within the first few months of their service. If the training proves to be a motivating factor for the volunteers, the organisation will be more likely to retain the new volunteers, but adverse reactions to the training may result in volunteers leaving the organisation.

\section{Motivations to Volunteer}

Sitting below the main research questions were three sub-questions. The first subquestion was: What were the volunteers' primary motivations to volunteer? Volunteers from both organisations identified altruism as the most important motivation, and either the acquisition of knowledge and skills, or the utilisation of previously acquired knowledge and skills, as another important motivation. The career motivation was often prominent, but also the most specific, meaning that it was either rated by volunteers as very important or not very important all. Other motivations that were significant included the influence of others, interactions with peers, personal challenge, and camaraderie. An important moderating factor through a number of these motivations appeared to be the stage of life that volunteers were in. Additionally, second-hand accounts of a number of volunteers who did not participate in the research were given, and suggested that other motivations such as the alleviation of loneliness and filling in of time were valid, but under-reported. 


\begin{abstract}
Altrusim
Previous research has suggested that altruism rarely, if ever, exists as a motivation for volunteers in isolation (Clary, Snyder, \& Stukas, 1998; Cnaan \& Goldberg-Glen, 1991). Rather, there exists a complex interplay between altruistic and self-oriented roles (Hustinx et al., 2010). This was the case for the volunteers interviewed within this study. Whilst all professed, at some point, to harbouring altruistic concern as a motivation for volunteering, this was always linked to a variety of other, more selforiented motives. For many, altruism was expressed as a helpful by-product of another more self-orientated goal. Burns et al. (2006) suggest that altruism may be a factor in all other motivations for volunteers, even those concerned with selfadvancement such as career and esteem (though to a lesser extent for these particular motivations than for others). The scope of this study did not allow enough data to examine this hypothesis closely, however, at a bare minimum it can at least be stated that altruism was present for each of the volunteers, alongside other motivations.
\end{abstract}

A more pessimistic way to look at the high altruistic rating, would be to suggest that the desire to make the world a better place is simply a socially desirable answer that people feel they ought to provide, or will make them look better. Or, to take this even further, an answer that they can provide to lessen any feelings of guilt or embarrassment about other self-orientated motives. The tendency for self-reported research to over-emphasise socially desirable answers is certainly an area that has been identified as a danger in previous research (Aydinli et al., 2016; Shye, 2010). And the tendency for volunteers to give socially desirable answers was supported by the informal observations of one of the experienced volunteers in Organisation A, and in the interview with one of the volunteers in Organisation B. I find it doubtful, given the openness of the interviewees, and their candidness about many other subjects, that any deception or misrepresentation was occurring at a conscious level. It has been suggested that helping behaviour, which is an area with close connections to volunteerism, involves a complex interplay of conscious and unconscious beliefs (Aydinli et al., 2014). A possible explanation could therefore be that volunteers are so attuned to hearing the most commonly given response as to why people volunteer (to "make the world a better place"), that they believe this to be the only genuine and proper answer to give. Thus, by deciding to volunteer, they assume at a conscious level that this is indeed their reason for doing it, even if there are other, perhaps sub- 
conscious, motivations at play which are more important to them. There are few people who are not happy to help others, especially if it can be accomplished through an activity they are already planning to undertake for other reasons. But gaining satisfaction from the act of helping people through an already pre-determined activity, and actually wanting to do the activity for the purpose of helping people, are two separate concepts that can possibly become confused. And by its very definition if there is another self-oriented motive present, the activity can no longer be purely altruistic anyway (Piliavin \& Charng, 1990).

\section{The Impacts of Age and Stage of Life}

The acquisition and use of skills and knowledge was expressed as an important motivation for the volunteers of both organisations. But this motivation was described in different ways by different volunteers, and these differences were strongly related to the volunteer's stage of life. For the younger volunteers this motivation was heavily driven by career goals, for those in their mid-life it was linked to the idea of personal challenge and development, and for those in retirement it was linked to the utilisation of knowledge, including that which was previously learnt.

Previous research has noted the influence of age and stage of life upon volunteer motivation. The career motivation has been shown to have a negative correlation with age (Ferreira et al., 2012), and those over 50 have been found to be more highly motivated by altruism and less by career motives (Principi et al., 2012). The three volunteers from this research who exhibited a significant career motivation were all aged under 40, with two of them being aged under 20. It is unsurprising to find out that those who are younger are more likely to be motivated by career motives, than those who are entering into retirement. Yet this has significant implications for the training of volunteers, as training, and more specifically qualifications, are much more sought after by those with this particular motivation. This is not to say that the acquisition of knowledge is not valued by those who are older. To the contrary, the acquisition of new knowledge was highly valued by those who were retired, and actually developed into a strong ongoing motivation. But the formality and recognition of this learning was not as important as the relevance of the learning and the ability to apply learning into practice. 
Warren and Garthwaite (2015) noted a significant effect of milestone events for the volunteers within their research. In the present study, three significant milestones were noted by the volunteers: entrance into the workforce, children moving out of home, and retirement. A growing number of young people are volunteering in order to help them to prepare for a future career path, and this can enhance the chances of a better degree and job prospects (Wilson, 2012). As has been discussed previously, the primary motivation of the two youngest volunteers in this research was to gain skills and experience to help them to prepare for a future career. One of the middle-aged volunteers noted that children starting to move out of the house had freed up more free time in which to volunteer. Clearly the volunteer's description of the need for a fresh challenge was also linked to this milestone event. And lastly, retirement also provided the time, and impetus, for the most senior volunteers in this study to seek a volunteering activity. Rather than a fresh challenge, however, this was seen as a chance to utilise previous gained skills, and "give back".

\section{The Influence of Others}

It was somewhat surprising that the social function from the VFI Questionnaire was not rated more highly by the volunteers in this research, and particularly by those from Organisation A. It is understandable that the sense of camaraderie and peer support that emerged as such a strong feature of the ongoing motivation for these volunteers may not have contributed much to the VFI results, as the questionnaire is primarily aimed towards capturing the initial motivations of volunteers. However, this does not account for the influence of others, both as a legacy influence and a present day energiser, which emerged as a strong initial motivation for the volunteers of Organisation A. Possibly the legacy influence was interpreted by the volunteers as better expressed by the values function, as they associated it with the altruistic nature of the service given by their role models from the past. Or it may be that the low results are another by-product of socially desirable reporting, with volunteers unwilling to admit that they are motivated by the need for social contact, and/or approval of peers. Nevertheless, this result is still unexpected given the answers emerging from the interviews. It should also be noted that the legacy influence of past role models upon present day volunteers is a theme that has not emerged from within the current body of research, and may warrant further investigation. 
The influence of present day energisers is an interesting factor for those hoping to recruit new volunteers, and has been noted in previous research. Okun et al. (2015) suggest that religiosity is such a strong predictor of volunteer service because it is far more common for those within religious contexts to directly ask others to volunteer. This phenomenon has similarly been noted amongst teenage volunteers by Bautista (2015). Many of the volunteers within the present research sought encouragement or clarification from those within the volunteer organisation, or other trusted figures, before committing to volunteering. Seeking reassurance from someone with equivalent practical experience, appears to provide prospective volunteers with a sense of reassurance and confidence about the decision to enrol. A strong sense of camaraderie, and volunteers with a sense of purpose, could thus be a crucial element that is needed to attract potential volunteers, as their decision to volunteer may be influenced by the opinions of those already volunteering for the organisation.

\section{Loneliness and Other "Undesirable" Motivations}

The last significant theme to emerge from the interviews is the third-party descriptions of hidden motivations. The dangers of self-reported research have already been noted, and either because of this phenomenon, or possibly because of the small sample size for this research, there appears to be a number of motivations which have not been captured. These include those who volunteer because they are lonely, those who wish to do something with spare time, and those who want to enhance or protect their ego. Shye (2010) attempted to get around self-reporting bias by asking a sample of people (both volunteers and non-volunteers) how much a pre-devised set of 16 possible volunteer motivations rewards the individual. By giving answers for an unspecified individual, the respondent was free from any judgement that they may have felt in giving socially undesirable answers. Further research, utilising non selfreported methods, would help to give greater insight into whether these undesirable motivations are indeed presently being unreported.

It is also noted by Hustinx et al. (2010) that there is a general tendency within any discussion about volunteerism, to over-emphasise virtuous aspects. At times it seems that any talk of self-interested motivations runs against the true ethos of volunteerism. In reality, both organisations within this research did not particularly care whether a volunteer was volunteering to help make the world a better place, gain skills towards 
a future job, or just to get out of the house, as long as they volunteered! Certain motivations undoubtedly come with added risks for the organisation, such as the risk that those wanting a qualification will leave once they have received it. However, confronted with this danger at Organisation B, VF24 stated that the organisation was quite happy to accept volunteers with overtly stated career motivations, and help them gain a nationally recognised qualification, so long as they commit to a reasonable length of service. As suggested by Clary, Snyder, and Stukas (1998), there is a danger that selling the myth of truly altruistic volunteering motivations may indeed be alienating volunteers with entirely valid, but more self-oriented motivations. Discussing the values of self-oriented volunteer motivations more openly and frequently, may help to encourage people who are lonely, or looking for an emotional lift, that volunteering is a valid and worthy option for them.

\section{Volunteer Perceptions of the Training}

The second sub-question was: What were the volunteers' perceptions of the training that they are required to complete? There were a number of similarities in the responses of the two groups of volunteers, despite the varying demographics and different training approaches of the two organisations. Both groups of volunteers appreciated the training when it was relevant to their learning needs, developed skills that the volunteers found useful, and involved interaction with experienced volunteers within the organisation. Troubles arose when there were unclear or ill-defined expectations, inconsistent or divisive delivery, and little flexibility in the learning structures. When the content was deemed by the volunteers to be non-relevant, the training became little more than a "tick the box" process, which was particularly difficult for those with competing priorities (such as work or family). For one group of volunteers it was demoralising when their prior skills and knowledge were not recognised and utilised.

\section{Relevance of Learning Content}

It has been widely noted that volunteers will be most satisfied, and therefore willing to volunteer for longer, if their motivations to volunteer are met by their volunteer service (Clary \& Snyder, 1999; Douglas \& Rollins, 2007; Houle et al., 2005). Taking this a step further, it has also been suggested that training is most likely to be seen as a 
benefit for a volunteer if it relates directly to their motivations for volunteering (Newton et al., 2014; Pells, 2008b). This was found to be true for a number of the volunteers interviewed for this research, in both a positive and negative sense. The two youngest volunteers, who both professed strong career motivations, were pleased with the practical skills and experience that they had gained to help them towards future careers, but one was frustrated at a lack of rapid progress towards a formal qualification. One of the adult literacy tutors who volunteered with the purpose of sharing a lifetime of knowledge and experience, was disappointed that this prior knowledge was not recognised, or utilised within the training programme (even as a participant). And several of the volunteers detailed how the training had opened their eyes to the difference that their volunteer service could make within their communities, thus satisfying their altruistic motivations. Through these responses it can be seen that where the volunteers' motivations were met by the training they were highly satisfied, but they were similarly frustrated when the training did not meet these motivations.

Perhaps more surprisingly, it was found that volunteer enthusiasm for a training programme could occur completely unrelated to motivations, if the volunteers' believed that the learning content was relevant and useful. The volunteers from Organisation A were happy with aspects of the training that helped them to work with adult learners, and understand better how to combat illiteracy, regardless of motivation. Likewise, all of the volunteers from Organisation B were quite happy to undertake training that they believed would keep them safe, and were willing to come outside of normal training hours if it helped them to familiarise themselves with key equipment. The main dissatisfactions expressed with the training for either organisation was not that the training did not relate to their volunteer motivations, but rather that it was irrelevant, disorganised, or had unclear expectations. Irrelevant content was a particular issue, and led to a "tick the box" mentality towards the training. Where the learning content was relevant and helpful however, the training was appreciated regardless of how relevant it was to the volunteers' primary motivations. 


\section{Overcoming Learning Obstacles}

A number of aspects of the training that the volunteers found troubling would be risks for any training activity. Lack of clear structures, unclear assessment guidelines, inconsistent delivery, and a lack of learner centred learning, are problems that would pose an issue for training in any workplace. However, because of the unique characteristics of volunteers, the risk of many of these potential training issues is even greater than in other environments. Time is already noted as a key training barrier for volunteers, as many of these volunteers are already fitting their volunteer service around family, work, and other social commitments (Attewell, 2009; Pells, 2008a; Warren \& Garthwaite, 2015). Additional commitment in the form of training or assessments, may force them to rethink their commitment to the volunteer organisation. This is a risk for volunteer organisations who may already be understaffed, and unable to offer the same enticements that a regular workplace could, such as promotion and wage increases, It is crucial that volunteers do not feel that their time is being wasted by pointless exercises, or inefficient systems. Noe (1986) notes that the motivation to learn is influenced by accurate assessment, the ability to master content with reasonable effort, and being provided with sufficient resources by the workplace to achieve learning goals. In the training programmes assessed by this research, there were clearly issues with some of these aspects which affected the learning motivation of the volunteers.

\section{Peer Learning with Experienced Volunteers}

An effective aspect of the training programmes for both Organisations was the utilisation of experienced volunteers in peer learning with the new recruits. Within Organisation A this was incorporated into the training programme itself, particularly in relation to the informal training aspects. The use of peer learning is a proven method of training volunteers (Douglas \& Rollins, 2007), and in the case of Organisation A it can be seen that in addition to generating good learning outcomes, it also helped to build camaraderie and integrate the new recruits into the squad. Effective training requires organisational support after the learning event (Boud \& Hager, 2012; Dailey, 1984; Eraut, 2004), and this occurred via the use of peer support within Organisation B. 


\section{Individualisation and Recognition of Prior Knowledge and Skills}

A number of the issues with the training stemmed from a lack of flexibility within the learning programmes which treated all learners as being equal, despite the fact that the volunteers came from vastly different backgrounds and harbour a wide variety of motivations. Whilst it is certainly easier for an organisation to create a single training programme for all volunteers, this approach may not lead to ideal learner outcomes. Volunteers with a high career motivation will most likely desire a robust training programme that leads to recognised qualifications, whereas an altruistically driven volunteer with a busy work and family life may struggle to keep up with the assessment demands that this may require. Likewise, a volunteer with a wealth of previous experience may not believe it worth their time and energy to complete new training at a low level, but a young volunteer with little previous education may find the demands of a programme at this same level to be daunting. This is a tricky balance for any volunteer organisation to manage, juggling the competing needs and motivations of a wide range of potential volunteers. From the responses of the interviews, however, there were few attempts at all to individualise, or even adjust, the training programme to meet learner needs. Organisations that can incorporate flexibility into their training will undoubtedly develop more satisfied and effective volunteers.

\section{External Influences}

It has already been noted that there can be a number of competing factors underlying the training programmes that volunteers are required to complete. It is therefore surprising to find little empirical research into the most effective methods of volunteer training (Huang et al., 2014). Presumably, most training providers rely upon training methods used within the regular workforce, despite the significant differences in profiles and motivations between the regular workforce and volunteers. Nonetheless, besides the desire to equip volunteers and keep them safe, which should always be the first priority, other competing factors are beginning to emerge more prominently as training priorities for volunteer organisations. In particular it is becoming increasingly important within New Zealand for training to be externally recognised, and generally this requires for the training to credit towards a qualification on the NZQF. This is partly for the benefit of the volunteers (so that they come out of the training with a 
recognised qualification which they can use elsewhere if they wish) but also for the benefit of the organisation, to demonstrate to stakeholders and funders that they have stringent and successful training procedures. The tension between serving the best outcomes of learners and appeasing outcomes-based funding structures is a growing concern within the wider educational sector in New Zealand (Chan, 2016, Pells 2008a). When the focus of the training becomes more about what is required to complete a qualification than what is required to equip the volunteers to be effective and safe in their role, this learning will be seen as irrelevant by most volunteers, unless they have a strong career related motivation.

The shift towards externally recognised training packages can work reasonably well within a workplace environment, where a high proportion of employees have reasonable levels of ambition to progress further in their career paths, and incentives such as promotions and pay increases can be gained through higher levels of training and qualifications. But volunteers are more likely than employees to be driven by non-career related motivations, such as altruistic or social reasons, for which training in and of itself has little relevance. For training to be perceived as being valuable for these volunteers it must either relate to their motivations for volunteering (e.g. utilises lots of group work for those with social motivations), or make them more effective in their volunteer activities. This becomes problematic when some of the most effective teaching methods for volunteers, are not a part of formal training systems. For example, the informal relationships between the new recruits and experienced volunteers were very effective for both organisations, but did not play a large role in either of the formal training systems. The shift towards the utilisation of national qualifications has led to less contextualised content and assessment (as by their design the national qualifications are required to be as general as possible to cover as many contexts as possible). This issue goes further than the volunteer sector, with Battersby (1999) arguing that the market based principles that were used to shape many of the reforms of the New Zealand education system led to a technocratic approach which treats professionals as a homogenous group. Whilst, a skilled tutor may be able to utilise generic qualifications so that they relate back to specific contexts, this does not always occur. The outcome of non-contextualised training is the "tick the box" mentality of doing what needs to be done to get through the training. The gap between 
generic concepts and the learner's context is not bridged, and the volunteer interprets the learning as irrelevant, and a waste of time.

\section{Links between Motivation and Training}

The final sub-question was: have the volunteers' motivations shifted since they started as a volunteer, either because of the training, or any other factor? This question had the intention of joining the two threads of volunteer motivation and volunteer training together. Almost all of the motivational factors which had developed since volunteers joined the organisation, either came as a result of the training, or had the potential to be enhanced by training activities. But interestingly, the aspects of training that the volunteers appreciated were not always related to their initial motivations. Volunteers were motivated by the training if it either related positively to their motivations to volunteer, or helped them to be more effective and safe in their volunteer role. Negative perceptions of the training, which had the potential to be de-motivators, were factors that either did not relate well to the volunteers' motivations, or that they perceived as being irrelevant.

\section{Factors that Enhanced Volunteer Motivation}

When asked about the motivations that had developed for the volunteers since joining the volunteer organisation, almost all of the motivations that were noted had some form of existing or potential link to training activities. The volunteers noted the motivations of intellectual challenge, and the development of new skills, which clearly have the potential to be developed through training. It must be noted, however, that this was not always the case, and there were a number of examples given where these motivations were developed quite independently of scheduled training activities, such as the adult literacy tutor who conducted independent research into learning challenges, and the firefighters who met with other more experienced firefighters in their spare time. The inspirational insight that was an important developed motivation for the literacy tutors, was for one of the volunteers introduced by the stories told during the training block course. And the peer relationships and camaraderie that were such strong features of the ongoing motivations of the firefighters, were at least partly fostered by the learning partnerships between experienced volunteers and new recruits, which were initially developed during the informal training nights. 
Identifying and integrating developing motivational factors within the training programmes of volunteer organisations can only help to increase the likelihood of new volunteers staying within the organisation. These motivations would probably look different from organisation to organisation (and even volunteer to volunteer), but using the training programmes detailed by this research as an example, there could be a number of potential changes to the training programmes which would possibly enhance these motivations further. For example, allowing the adult literacy tutors to investigate areas of interest for their assessments and then share them back to the group, would help to foster intellectual challenge, the ability to share existing (or in this case developed) skills and knowledge, and to create inspirational insight for others in the organisation. This would of course require some creativity and flexibility in training design, and there would undoubtedly be practical hurdles to overcome, however, the findings of this research suggest that when volunteer motivations are developed within a training programme, the training can create high levels of commitment towards the organisation by the volunteer.

\section{Training as a Motivator}

Overall, by far the most important aspect of training for any of the volunteers, was that it was relevant to the learning needs of the volunteer. Existing research stating that training should relate to volunteer motivation has already been noted (Newton et al., 2014; Pells, 2008b). And whilst matching the training to key motivations did show positive effects in this research, it was more important for the volunteers that the training was relevant and useful. Thus, for the firefighters, whilst none of them expressed that a motivation to volunteer was the chance to work in a safe work environment, all of them appreciated understanding the safety requirements that they learnt through the training. This is obviously connected to the fact that they are operating in dangerous environments, and thus appreciate the need to understand how to keep themselves (and others) safe. When the training helped them to understand this effectively, they appeared to be positive about both the training, and their volunteering, regardless of prior motivation. This makes sense in the context of this research, where each of the volunteers harboured some form of altruistic motivation. If volunteers are motivated to help people through their service, anything that helps 
them to be more effective in their service (and therefore more effective in helping others) will surely help to satisfy this motivation.

This should not discount the positive effect that linking motivation to training can have. Those who entered volunteering with a hope of personal challenge expressed satisfaction when they were stretched by the training. Others who entered volunteering with a high career motivation, appreciated the skills and knowledge that they received (including some which were unrelated to their volunteering activities) which helped them to advance their career goals. Though there were no volunteers from within the sample who wanted to alleviate loneliness, it is safe to assume that if these volunteers were to be interviewed, they would express more fulfilment from group learning activities than learning activities with no physical social contact, such as an online learning module.

In summary, the training activities helped to motivate volunteers when they either related positively to their motivations to volunteer, or made them more effective in their volunteering activities.

\section{Training as a Potential De-Motivator}

It follows from the previous section, that training acted as a de-motivator when it was seen to be irrelevant, or unrelated to their volunteer motivations. The training was deemed to be irrelevant by the volunteers when it was perceived to have little relevance to the volunteer's context, and thus became a "ticking the box" exercise. Volunteers noted additional de-motivation factors such as; a lack of learning structures, unclear or unrealistic expectations (particularly when this put pressure on other commitments such as work and family), and prior knowledge and experience not being recognised. 


\section{Conclusion}

This research has explored the motivations of volunteers from two contrasting volunteer organisations, and sought to find out whether these motivations were changed or enhanced by the training programmes that they were required to undertake. It was found that the motivational functions of the two groups of volunteers were very similar, with the values function and understanding function, rating the highest, and the careers function as the most variable. How these motivational functions were expressed, however, was different across the two groups, primarily in relation to demographic differences. For example, whilst younger volunteers tended to hold the understanding function due to a desire to obtain new knowledge, older volunteers tended to harbour the same motivational function as a way to utilise existing skills and knowledge. As well as age, the motivational functions were also affected by key milestone life events such as entering the work force, children leaving home, and retirement. Altruism (the values motivational function) was expressed as the most important motivation overall, but it was always present alongside other more self-oriented motivations. It is unclear whether this is because altruism often works alongside and moderates other motivations, or if this was as a result of socially desirable reporting. The absence of a number of motivations such as the alleviation of loneliness, and filling in time, could suggest that these may be motivations that people are less likely to disclose.

Volunteers appreciated the training when they found the content to be relevant and useful, there was good interaction with experienced peers, and they felt challenged by it (but not challenged too far). It was found to be unhelpful when expectations were unclear, prior learning and experience was not recognised, and the content was based on issues that were thought to be irrelevant to the practice of the volunteers. Overall the motivations of the volunteers were enhanced by the training when they either found the training to be relevant and useful to their volunteer role, or it related well to their motivations to volunteer.

\section{Recommendations for Volunteer Organisations}

It is apparent from the research available that volunteers differ greatly as to their reasons for volunteering with an organisation. By acquiring information about the 
motivations of their volunteers, organisations will be better equipped to ensure that both their volunteer environments, and the training that they provide, are suited to the motivations of their volunteers. The utilisation of a tool such as the VFI Questionnaire, or well-crafted interview questions, can provide useful information. But those gathering the information should be aware of the tendency for respondents to over-emphasise socially desirable responses (such as altruism) and downplay others that are less favourable (such as alleviating loneliness). It should also be acknowledged that motivations are constantly changing, and may alter as a result of the volunteer's service, or any training that they undertake.

In designing training programmes, organisations should ensure that the training material is highly relevant to the volunteer's practice, and contextualised to their setting. As much as possible, there should be flexibility within the system to allow volunteers to explore areas of interest and need, and utilise existing skills and knowledge. The use of experienced volunteers to help to guide new recruits is a factor that should be incorporated into training programmes wherever possible. And, as altruism is one of the most common and highly rated motivations for volunteers, those designing training programmes should always attempt to demonstrate how the training will help the volunteers to help others, such as through the sharing of inspirational stories and experiences.

\section{Limitations of the Research}

This study has a number of possible limitations which may have influenced the findings. Due to the small sample size, it cannot be assumed that any of the findings are representative of the views of all of the volunteers from either of the organisations represented within this study. As noted in the literature review, culture and ethnicity have a significant impact upon volunteering, both within New Zealand and internationally. All participants in this research were either British European, or New Zealand European, and this lack of cultural diversity may have limited the findings. It is acknowledged that there are a number of potential motivations which were not identified by those participating in the research, and the views of volunteers with these differing motivations or experiences may have changed the findings significantly. The usage of self-reported motivations may have skewed the motivational findings towards motivations which are more socially desirable (such as 
altruism) at the expense of the less social desirable motivations (such as the alleviation of loneliness). This bias may have affected the research at two levels, the extent to which those who participated felt able to fully share these motivations, but also the exclusion of volunteers who did not wish to participate in the research due to not wanting to disclose motivations which they felt to be embarrassing. Most importantly, the views of those who withdrew from volunteering activities, and/or training events, are an important source of feedback that is not captured here (other than through second-hand anecdotal evidence).

\section{Recommendations for Further Research}

Six avenues for further research are suggested which would help to further explore the interactions between volunteer motivations and training.

The majority of research into volunteer motivations has utilised self-reporting methods which may skew findings towards those that are more socially desirable. More research utilising alternative techniques, such as those utilised by Shye (2010), would help to explore whether social desirability is a significant factor in the majority of existing research into volunteer motivations.

A significant contributor to the motivations of the volunteers in this study, which has not been identified previously, is the influence of past role models. The impact of these role models, and how this legacy impacts upon the present-day motivations of volunteers, is another area worthy of further investigation.

It is difficult to locate volunteers who have left volunteer organisations, let alone convince them to participate in research, however, these past volunteers may have valuable perspectives, particularly regarding barriers towards volunteer training. Research which explores the views of those who have withdrawn from volunteer service or training programmes would help to give a greater understanding of why this may occur.

There is a lack of substantial research into the most effective methods of training volunteers. Exploration into the specific training needs of volunteers, and what differences there are between training regular employees and volunteers, would be highly valuable to the volunteer sector. 
There is a suspicion that the growing professionalisation of the volunteer sector is excluding a number of volunteers who may otherwise have given useful service. Research into this training shift (including those who have left or decided not to enrol as a volunteer) may help to identify whether an increase in professional requirements within the sector is proving to be a barrier to participation for potential volunteers.

Lastly, it has been noted in this research that there is an increasing move towards utilising nationally recognised qualifications within volunteer training. Research into the effects that this shift is having on the sector would help to identify the positive and negative aspects of nationally recognised training, and thus provide valuable information for volunteer organisations to consider. 


\section{References}

Alexander, A., Kim, S., \& Kim, D. (2015). Segmenting volunteers by motivation in the 2012 London Olympic Games. Tourism Management, 47, 1-10. https://doi.org/10.1016/j.tourman.2014.09.002

Arthur, W., Bennett, W., Edens, P. S., \& Bell, S. T. (2003). Effectiveness of training in organizations: A meta-analysis of design and evaluation features. Journal of Applied Psychology, 88(2), 234-245. https://doi.org/10.1037/0021-9010.88.2.234

Attewell, A. (2009). Participation of New Zealand Fire Service volunteer firefighters in a training and progression system. Unpublished Manuscript.

Aydinli, A., Bender, M., Chasiotis, A., Cemalcilar, Z., \& van de Vijver, F. J. R., (2014). When does self-reported prosocial motivation predict helping? The moderating role of implicit prosocial motivation. Motivation and Emotion; New York, 38(5), 645-658. https://doi.org/http://dx.doi.org.helicon.vuw.ac.nz/10.1007/s11031-014-9411-8

Aydinli, A., Bender, M., Chasiotis, A., van de Vijver, F. J. R., Cemalcilar, Z., Chong, A., \& Yue, X. (2016). A cross-cultural study of explicit and implicit motivation for longterm volunteering. Nonprofit and Voluntary Sector Quarterly, 45(2), 375-396. https://doi.org/10.1177/0899764015583314

Babbage, S. E. (1990). Research into the training of volunteers (Master's thesis). Retrieved from http://restrictedarchive.vuw.ac.nz//handle/123456789/5619

Bandura, A. (1986). Social foundations of thought and action: A social cognitive theory. Englewood Cliffs, N.J.: Prentice-Hall.

Battersby, D. (1999). The learning organization and CPE: Some philosophical considerations. The Learning Organization, 6(2), 58-62. https://doi.org/10.1108/09696479910262578

Bautista, R. (2015). Examining learner-centered training with teen volunteer staff at an aquarium (Master's $\quad$ Thesis). $\quad$ Retrieved from http://search.proquest.com/docview/1659817391/

Baxter, P., \& Jack, S. (2009). Qualitative case study methodology: Study design and implementation for novice researchers. Qualitative Report, 13(4), 544-559.

Boud, D., \& Hager, P. (2012). Re-thinking continuing professional development through changing metaphors and location in professional practices. Studies in Continuing Education, 34(1), 17-30. https://doi.org/10.1080/0158037X.2011.608656

Boyatzis, R. E. (1998). Transforming qualitative information: Thematic analysis and code development. Thousand Oaks, CA: Sage Publications.

Braun, V., \& Clarke, V. (2006). Using thematic analysis in psychology. Qualitative Research in Psychology, 3(2), 77-101. https://doi.org/10.1191/1478088706qp063oa

Burns, D. J., Reid, J. S., Toncar, M., Fawcett, J., \& Anderson, C. (2006). Motivations to volunteer: The role of altruism. International Review on Public and Non - Profit Marketing, $3(2)$, 79-91. https://doi.org/http://dx.doi.org.helicon.vuw.ac.nz/10.1007/BF02893621 
Burns, D. J., Reid, J., Toncar, M., Anderson, C., \& Wells, C. (2008). The effect of gender on the motivation of members of Generation Y college students to volunteer. Journal of Nonprofit \& Public Sector Marketing, 19(1), 99-118. https://doi.org/10.1300/J054v19n01_05

Callero, P., Howard, J., \& Piliavin, J. (1987). Helping behavior as role behavior: Disclosing social structure and history in the analysis of prosocial action. Social Psychology Quarterly, 50(3), 247.

Cappellari, L., \& Turati, G. (2004). Volunteer labour supply: The role of workers' motivations. Annals of Public and Cooperative Economics, 75(4), 619-643. https://doi.org/10.1111/j.1467-8292.2004.00265.x

Cervero, R. M. (2001). Continuing professional education in transition, 1981-2000. International Journal of Lifelong Education, 20(1-2), 16-30. https://doi.org/10.1080/09638280010008282

Chacón, F., Vecina, M. L., \& Dávila, M. C. (2007). The three-stage model of volunteers' duration of service. Social Behavior and Personality, 35(5), 627-642.

Chan, S. (2016). New Zealand's move to graduate-profile framed qualifications: Implications, challenges and the occupational identity solution. International Journal of Training Research, 14(1), 5-18. https://doi.org/http://dx.doi.org.helicon.vuw.ac.nz/10.1080/14480220.2016.1156315

Charbonneau, J., Brennan, M., \& Hercus, A. (2006). New Zealand volunteers: A snapshot. Retrieved from http://www.communityresearch.org.nz/

Chou, K. (1996). The Rushton, Chrisjohn and Fekken self-report Altruism scale: A Chinese translation. Personality and Individual Differences, 21(2), 297-298.

Clark, J. (1997). Educational research: Philosophy, politics, ethics. Palmerston North: ERDC Press.

Clary, E., \& Snyder, M. (1999). The motivations to volunteer: Theoretical and practical considerations. Current Directions in Psychological Science, 8(5), 156-159. https://doi.org/10.1111/1467-8721.00037

Clary, E., Snyder, M., Ridge, R. D., Copeland, J., Stukas, A. A., Haugen, J., \& Miene, P. (1998). Understanding and assessing the motivations of volunteers: A functional approach. Journal of Personality and Social Psychology, 74(6), 1516-1530.

Clary, E., Snyder, M., \& Stukas, A. (1998). Service-learning and psychology: Lessons from the psychology of volunteers' motivations. In R. G. Bringle \& D. K. Duffy (Eds.), With service in mind: Concepts and models for service-learning in psychology (pp. 35-50). Washington, DC: American Psychological Association. https://doi.org/10.1037/10505-002

Clary, E., Snyder, M., \& Stukas, A. A. (1996). Volunteers' motivations: Findings from a national survey. Nonprofit and Voluntary Sector Quarterly, 25(4), 485-505. https://doi.org/10.1177/0899764096254006

Cnaan, R. A., \& Goldberg-Glen, R. S. (1991). Measuring motivation to volunteer in human services. The Journal of Applied Behavioral Science, 27(3), 269.

Cnaan, R. A., Handy, F., \& Wadsworth, M. (1996). Defining who is a volunteer: Conceptual and empirical considerations. Nonprofit and Voluntary Sector Quarterly, 25(3), 364383. https://doi.org/10.1177/0899764096253006 
Creswell, J. W., Hanson, W. E., Plano Clark, V. L., \& Morales, A. (2007). Qualitative research designs: Selection and implementation. Counseling Psychologist, 35(2), 236-264. https://doi.org/10.1177/0011000006287390

Dailey, N. (1984). Adult Learning and Organizations. Training and Development Journal, 38(12). 64-68.

Daley, B. J. (2001). Learning and professional practice: A study of four professions. Adult Education Quarterly, 52(1), 39-54. https://doi.org/10.1177/074171360105200104

Davis Smith, J. (2000). Volunteering and social development. Voluntary Action, 3(1).

Davis Smith, J. (2001). Volunteering, capital of the future? The UNESCO Courier, 54(6), 2021.

de Haas, R. (2012). 2011 Volunteer sustainability report. Unpublished Manuscript.

Deci, E. L. (1971). Effects of externally mediated rewards on intrinsic motivation. Journal of Personality and Social Psychology, 18(1), 105-115. https://doi.org/10.1037/h0030644

Deci, E. L., \& Ryan, R. M. (2008). Self-determination theory: A macrotheory of human motivation, development, and health. Canadian Psychology, 49(3), 182-185. https://doi.org/10.1037/a0012801

Degli Antoni, G. (2009). Intrinsic vs. extrinsic motivations to volunteer and social capital formation. Kyklos, 62(3), 359-370. https://doi.org/10.1111/j.1467-6435.2009.00440.x

Denzin, N. K., \& Lincoln, Y. S. (2008). Strategies of qualitative inquiry. (3rd ed.). Los Angeles: Sage Publications.

Deslandes, M., \& Rogers, L. (2008). A volunteer training framework. Australian Journal of Adult Learning, 48(2), 354-368.

Do Paço, A., Agostinho, D., \& Nave, A. (2013). Corporate versus non-profit volunteering: Do the volunteers' motivations significantly differ? International Review on Public and Non - Profit Marketing, 10(3), 221-233. https://doi.org/http://dx.doi.org.helicon.vuw.ac.nz/10.1007/s12208-013-0101-0

Douglas, C., \& Rollins, R. (2007). Motivations, training and supervision of volunteers in conservation. Environments, 35(1), 79-89.

Duxfield, A. (2016). A Volunteering New Zealand summary report: State of the world's volunteerism report. Wellington, New Zealand: Volunteering New Zealand. Retrieved from

https://www.dia.govt.nz/diawebsite.nsf/Files/About_volunteering_NZ/\$file/About_vo lunteering_NZ.pdf

Ellis, C., Holmes, K., O’Dwyer, L., Oppenheimer, M., Paull, M., \& Webber, C.. (2014). Volunteering Australia's national review of the definition of volunteering in Australia. Volunteering Australia. Retrieved from http://www.volunteeringaustralia.org/

Eraut, M. (2004). Informal learning in the workplace. Studies in Continuing Education, 26(2), 247-273. https://doi.org/10.1080/158037042000225245

Erez, A., Mikulincer, M., van Ijzendoorn, M. H., \& Kroonenberg, P. M. (2008). Attachment, personality, and volunteering: Placing volunteerism in an attachment-theoretical framework. Personality and Individual Differences, 44(1), 64-74. https://doi.org/10.1016/j.paid.2007.07.021 
Fényes, H. (2015). Effect of religiosity on volunteering and on the types of volunteering among higher education students in a cross-border central and Eastern European region. Acta Universitatis Sapientiae. Social Analysis; Cluj-Napoca, 5(2), 181-203.

Ferreira, M. R., Proença, T., \& Proença, J. F. (2012). Motivation among hospital volunteers: An empirical analysis in Portugal. International Review on Public and Non - Profit Marketing, $9(2)$,

$137-152$. https://doi.org/http://dx.doi.org.helicon.vuw.ac.nz/10.1007/s12208-012-0083-3

Finkelstien, M. A. (2009). Intrinsic vs. extrinsic motivational orientations and the volunteer process. Personality and Individual Differences, 46(5-6), 653-658. https://doi.org/10.1016/j.paid.2009.01.010

Ford, M. E. (1992). Motivating humans: Goals, emotions and personal agency beliefs. London: Sage Publications.

Fortier, J., Auger, D., \& Froment-Prévosto, C. (2007). The motivations and satisfactions of youth volunteers in leisure and sport activities in Quebec: The perspective of the volunteer and the organisation. Voluntary Action, 8(3), 79-97.

Ginsberg, M. (2005). Cultural diversity, motivation, and differentiation. Theory into Practice, $44(3), 218-225$.

Grimm, K. E., \& Needham, M. D. (2012). Moving beyond the 'I' in motivation: Attributes and perceptions of conservation volunteer tourists. Journal of Travel Research, 51(4), 488-501. https://doi.org/10.1177/0047287511418367

Grönlund, H., Holmes, K., Kang, C., Cnaan, R. A., Handy, F., Brudney, J., Zrinscak, S. (2011). Cultural values and volunteering: A cross-cultural comparison of students' motivation to volunteer in 13 countries. Journal of Academic Ethics, 9(2), 87-106. https://doi.org/http://dx.doi.org.helicon.vuw.ac.nz/10.1007/s10805-011-9131-6

Grünbaum, N. N. (2007). Identification of ambiguity in the case study research typology: What is a unit of analysis? Qualitative Market Research: An International Journal, 10(1), 78-97. https://doi.org/10.1108/13522750710720413

Hackl, F., Halla, M., \& Pruckner, G. (2007). Volunteering and Income - The Fallacy of the Good Samaritan? Kyklos, 60(1), 77-104.

Handy, F., \& Srinivasan, N. (2005). The demand for volunteer labor: A study of hospital volunteers. Nonprofit and Voluntary Sector Quarterly, 34(4), 491-509. https://doi.org/10.1177/0899764005278037

Holmes, K., \& Slater, A. (2012). Patterns of voluntary participation in membership associations: A study of UK heritage supporter groups. Nonprofit and Voluntary Sector Quarterly, 41(5), 850-869. https://doi.org/10.1177/0899764011420881

Houle, B. J., Sagarin, B. J., \& Kaplan, M. F. (2005). A functional approach to volunteerism: Do volunteer motives predict task preference? Basic and Applied Social Psychology, 27(4), 337-344. https://doi.org/10.1207/s15324834basp2704_6

Huang, Y., Strawderman, L., Babski-Reeves, K., Ahmed, S., \& Salehi, A. (2014). Training effectiveness and trainee performance in a voluntary training program: Are trainees really motivated? Nonprofit and Voluntary Sector Quarterly, 43(6), 1095-1110. https://doi.org/10.1177/0899764013506787 
Hudson, S., \& Inkson, K. (2006). Volunteer overseas development workers: The hero's adventure and personal transformation. Career Development International, 11(4), 304-320.

https://doi.org/http://dx.doi.org.helicon.vuw.ac.nz/10.1108/13620430610672522

Hustinx, L., Cnaan, R. A., \& Handy, F. (2010). Navigating theories of volunteering: A hybrid map for a complex phenomenon. Journal for the Theory of Social Behaviour. https://doi.org/10.1111/j.1468-5914.2010.00439.x

Internal Affairs. (2016). Briefing to the incoming minister: Community and voluntary sector. No author. Retrieved from https://www.dia.govt.nz/diawebsite.nsf/wpg_URL/Resource-material-Briefings-toIncoming-Ministers-Index?OpenDocument

Jasperse, M., Herst, P., \& Kane, J. (2012). Evaluation of the training and support received by facilitators of a cancer education and support programme in New Zealand. European Journal of Cancer Care, 21(2), 224-232. https://doi.org/10.1111/j.13652354.2011.01303.x

Jiménez, M. L. V., Fuertes, F. C., \& Abad, M. J. S. (2010). Differences and similarities among volunteers who drop out during the first year and volunteers who continue after eight years. The Spanish Journal of Psychology; Madrid, 13(1), 343-52.

Johnson, B., \& Christensen, L. B. (2012). Educational research: quantitative, qualitative, and mixed approaches (4th ed.). Thousand Oaks, CA: SAGE Publications.

Kenwright, H. (2000). Volunteering to learn: Approaches to educational provision for volunteers and their clients. Voluntary Action, 3(1). Retrieved from http://www.ivr.org.uk/component/ivr/volunteering-to-learn

Kinnane, N. A., Waters, T., \& Aranda, S. (2011). Evaluation of a pilot 'peer support' training programme for volunteers in a hospital-based cancer information and support centre. Supportive Care in Cancer, 19(1), 81-90. https://doi.org/http://dx.doi.org.helicon.vuw.ac.nz/10.1007/s00520-009-0791-3

Konrath, S., Fuhrel-Forbis, A., Lou, A., \& Brown, S. (2012). Motives for volunteering are associated with mortality risk in older adults. Health Psychology, 31(1), 87-96. https://doi.org/http://dx.doi.org.helicon.vuw.ac.nz/10.1037/a0025226

Laczo, R. M., \& Hanisch, K. A. (1999). An examination of behavioral families of organizational withdrawal in volunteer workers and paid employees. Human Resource Management Review, 9(4), 453-477. https://doi.org/10.1016/S1053-4822(99)00029-7

Lavenburg, P., \& Bernt, F. M. (2012). Training and supporting hospice volunteers: A regional survey. American Journal of Hospice and Palliative Medicine, 29(5), 355361. https://doi.org/10.1177/1049909111423527

Literacy Aotearoa. (n.d.). Statistics. Retrieved from http://www.literacy.org.nz/who-weare/statistics

Lo Presti, A. (2013). The interactive effects of job resources and motivations to volunteer among a sample of Italian volunteers. Voluntas, 24(4), 969-985. https://doi.org/http://dx.doi.org.helicon.vuw.ac.nz/10.1007/s11266-012-9288-7

Lukka, P., \& Locke, M (2003). Faith and voluntary action: Community, values and resources. London: Institute for Volunteering Research. Retrieved from http://www.ivr.org.uk/component/ivr/faith-and-voluntary-action 
Manetti, G., Bellucci, M., Como, E., \& Bagnoli, L. (2015). Investing in volunteering: Measuring social returns of volunteer recruitment, training and management. VOLUNTAS: International Journal of Voluntary and Nonprofit Organizations, 26(5), 2104-2129. https://doi.org/10.1007/s11266-014-9497-3

McCudden, J. (2000). What makes a committed volunteer? Research into the factors affecting the retention of volunteers in home-start. Voluntary Action, 2(2), 59-75.

McDonald, C., \& Warburton, J. (2001). The invisibility of volunteers and the need for research: An Australian perspective. Voluntary Action, 4(1), 1-15.

Merriam, S. B. (1998). Qualitative research and case study applications in education. San Francisco: Jossey-Bass.

Merriam, S. B. (2009). Qualitative research a guide to design and implementation. San Francisco: Jossey-Bass. Retrieved from http://helicon.vuw.ac.nz/login?url=http://www.VUW.eblib.com/EBLWeb/patron/?tar get $=$ patron\&extendedid=P_1662771_0

Ministry of Education. (2014). Ministry of Education statement of intent 2014 - 2018. Wellington, Author: Retrieved from http://www.education.govt.nz/assets/Documents/Ministry/Publications/Statements-ofintent/2014SOI.pdf

Moses, K., \& Strathdee, R. (2007). The changing role of industry training organisations in New Zealand. New Zealand Annual Review of Education, 17, 59-72.

Muckaden, M., \& Pandya, S. (2016). Motivation of volunteers to work in palliative care setting: A qualitative study. Indian Journal of Palliative Care, 22(3), 348-353. https://doi.org/http://dx.doi.org.helicon.vuw.ac.nz/10.4103/0973-1075.185083

Murphy, G. A., \& Calway, B. A. (2008). Professional development for professionals: beyond sufficiency learning. Australian Journal of Adult Learning, 48(3), 424-444

Mykletun, R. J., \& Himanen, K. (2016). Volunteers at biking race events: Antecedents of commitment and intention to remain volunteering at future events. Sport, Business and Management: An International Journal, 6(3), 246-273. https://doi.org/10.1108/SBM-12-2014-0051

Newton, C., Becker, K., \& Bell, S. (2014). Learning and development opportunities as a tool for the retention of volunteers: A motivational perspective. Human Resource Management Journal, 24(4), 514-530. https://doi.org/10.1111/1748-8583.12040

Nichols, G., Taylor, P., James, M., Holmes, K., King, L., \& Garrett, R. (2005). Pressures on the UK voluntary sport sector. Voluntas, 16(1), 33-50. https://doi.org/http://dx.doi.org.helicon.vuw.ac.nz/10.1007/s11266-005-3231-0

Noe, R. A. (1986). Trainees' attributes and attitudes: Neglected influences on training effectiveness. The Academy of Management Review, 11(4), 736-749. https://doi.org/10.2307/258393

Offstein, E. H., Chory, R. M., \& Childers Jr, J. S. (2015). A cross-cultural analysis of factors influencing choice in volunteering activities. Competitiveness Review, 25(1), 74-97.

Okun, M. A., O’Rourke, H. P., Keller, B., Johnson, K. A., \& Enders, C. (2015). Valueexpressive volunteer motivation and volunteering by older adults: Relationships with religiosity and spirituality. The Journals of Gerontology, 70(6), 860-870. https://doi.org/10.1093/geronb/gbu029 
Oosterman, J., Sedgwick, C., \& Grey, S. (2017). Education under pressure: The 2016 New Zealand tertiary education state of the sector survey. Tertiary Education Union Te Hautu Kahurangi o Aotearoa. Retrieved from http://teu.ac.nz/wpcontent/uploads/2017/03/Education-under-pressure-report-1.pdf

Pells, S. (2008a). Volunteer-related training in emergency services: Findings from a literature review. Wellington: NZIER. Retrieved from http://www.communityresearch.org.nz/

Pells, S. (2008b). Volunteer-related training in emergency services - improved training outcomes: Findings from qualitative research. Wellington: NZIER. Retrieved from http://www.communityresearch.org.nz/

Piggot-Irvine, E. (2006). Establishing criteria for effective professional development and use in evaluating an action research based programme. Journal of In-Service Education, 32(4), 477-496. https://doi.org/10.1080/13674580601024432

Piliavin, J. A., \& Charng, H.-W. (1990). Altruism: A review of recent theory and research. Annual Review of Sociology, 16, 27.

Piliavin, J. A., Grube, J. A., \& Callero, P. L. (2002). Role as resource for action in public service. Journal of Social Issues, 58(3), 469-485. https://doi.org/10.1111/00224537.t01-1-00027

Principi, A., Chiatti, C., \& Lamura, G. (2012). Motivations of older volunteers in three European countries. International Journal of Manpower, 33(6), 704-722. https://doi.org/http://dx.doi.org.helicon.vuw.ac.nz/10.1108/01437721211261831

Salehi, A., Strawderman, L., Huang, Y., Ahmed, S., \& Babski-Reeves, K. (2009). Effectiveness of three training delivery methods in a voluntary program. Proceedings of the Human Factors and Ergonomics Society Annual Meeting, 53(26), 1959-1963. https://doi.org/10.1177/154193120905302617

Senior, D. (2006). Stock-take of community organisations providing social services in Waitakere. Auckland: Ministry of Social Development. Retrieved from http://www.communityresearch.org.nz/

Shye, S. (2010). The motivation to volunteer: A systemic quality of life theory. Social Indicators Research, 98(2), 183-200. https://doi.org/10.1007/s11205-009-9545-3

Silverberg, K., Ellis, G., Backman, K., \& Backman, S. (1999). An identification and explication of a typology of public parks and recreation volunteers. World Leisure \& Recreation, 41(2), 30-34. https://doi.org/10.1080/10261133.1999.9674148

Simha, A., Topuzova, L. N., \& Albert, J. F. (2011). V for volunteer(ing): The journeys of undergraduate volunteers. Journal of Academic Ethics, 9(2), 107-126. https://doi.org/http://dx.doi.org.helicon.vuw.ac.nz/10.1007/s10805-011-9136-1

Skinner, B. F. (1954). The science of learning and the art of teaching. Harvard Educational Review, 24, 86-97.

Smith, K., Cordery, C., \& Dutton, N. (2010). Managers matter: Who manages New Zealand's volunteers? Wellington: Victoria University of Wellington. Retrieved from http://www.volunteeringnz.org.nz/

Snyder, M., \& Omoto, A. M. (2008). Volunteerism: Social issues perspectives and social policy implications. Social Issues and Policy Review, 2(1), 1-36. https://doi.org/10.1111/j.1751-2409.2008.00009.x 
Stewart, E., \& Weinstein, R. S. (1997). Volunteer participation in context: Motivations and political efficacy within three AIDS organizations. American Journal of Community Psychology, 25(6), 809-837. https://doi.org/10.1023/A:1022265213167

Strathdee, R. (2011). The implementation, evolution and impact of New Zealand's national qualifications framework. Journal of Education and Work, 24(3-4), 303-321. https://doi.org/10.1080/13639080.2011.584688

Strathdee, R. (2012). The social composition of VET in New Zealand. Globalisation, $\begin{array}{llll}\text { Societies } \quad \text { and } & \text { Education, }\end{array}$ https://doi.org/10.1080/14767724.2012.646882

Stukas, A. A., Worth, K. A., Clary, E. G., \& Snyder, M. (2009). The matching of motivations to affordances in the volunteer environment. Nonprofit and Voluntary Sector Quarterly, 38(1), 5-28. https://doi.org/10.1177/0899764008314810

Tamasese, T. K., Parsons, T. L., Sullivan, G., \& Waldegrave, C. (2010). A qualitative study into Pacific perspectives on cultural obligations and volunteering. The Family Centre Social Policy Research Unit. Retrieved from http://www.familycentre.org.nz/

Taylor, B., \& Garratt, D. (2010). The professionalisation of sports coaching: Relations of power, resistance and compliance. Sport, Education and Society, 15(1), 121-139. https://doi.org/10.1080/13573320903461103

Tennant, M. (2001). Governments and voluntary sector welfare: Historians' perspectives. Social Policy Journal of New Zealand, 147.

The Department of Internal Affairs. (2001). Report of the Ministerial Reference Group for International Year of Volunteers 2001. Wellington: Author. Retrieved from www.dia.govt.nz

Tollefson, N. (2000). Classroom applications of cognitive theories of motivation. Educational Psychology Review, 12(1), 63-83.

Urdan, T., \& Schoenfelder, E. (2006). Classroom effects on student motivation: Goal structures, social relationships, and competence beliefs. Journal of School Psychology, 44(5), 331-349. https://doi.org/10.1016/j.jsp.2006.04.003

Vaughan, K. (2005). The pathways framework meets consumer culture: Young people, careers, and commitment. Journal of Youth Studies, 8(2), 173-186. https://doi.org/10.1080/13676260500149261

Vocino, A., \& Polonsky, M. J. (2011). Volunteering for research: A test of the psychometric properties of the volunteer functions inventory with online panellists. International Journal of Public Opinion Research, 23(4), 508-521. https://doi.org/10.1093/ijpor/edr013

Volunteering New Zealand. (2014). Constitution of Volunteering New Zealand Incorporated. Retrieved from http://www.volunteeringnz.org.nz/wp-content/uploads/2014-11Constitution-of-Volunteering-New-Zealand-Incorporated-FINAL_signed.pdf

Volunteering New Zealand. (n.d.). Statistics on Volunteering. Retrieved from http://www.volunteeringnz.org.nz/policy/statistics/

Warren, J., \& Garthwaite, K. (2015). 'We are volunteers and that sometimes gets forgotten': Exploring the motivations and needs of volunteers at a healthy living resource centre in the North East of England. Perspectives in Public Health; London, 135(2), 102107. 
Wilson, J. (2012). Volunteerism research: A review essay. Nonprofit and Voluntary Sector Quarterly, 41(2), 176-212. https://doi.org/10.1177/0899764011434558

Wlodkowski, R., \& Ginsberg, M. (1995). A framework for culturally responsive teaching. Educational Leadership, 53(1), 17-21.

Wlodkowski, R. J. (1997). Motivation with a mission: Understanding motivation and culture in workshop design. New Directions for Adult and Continuing Education, 1997(76), 19-31. https://doi.org/10.1002/ace.7602

Yeung, A. B. (2004). The octagon model of volunteer motivation: Results of a phenomenological analysis. VOLUNTAS: International Journal of Voluntary and Nonprofit

Organizations, $15(1)$ $21-46$. https://doi.org/10.1023/B:VOLU.0000023632.89728.ff

Yin, R. K. (2009). Case study research: Design and methods (4th ed.). Los Angeles: Sage Publications. 


\section{Appendices}

Appendix A - Organisational Information Sheet

Appendix B - Organisational Consent Form

Appendix C - Participant Information Sheet

Appendix D - Participant Consent Form

Appendix E - Interview Guide

Appendix F - VFI Questionnaire

Appendix G - VFI Questions by Motivational Function 


\section{Appendix A - Organisational Information Sheet}

Research Project Title: Training for Volunteers - a multiple case study investigating the influence of volunteer motivations in two training programmes.

Researcher: Phil Daly, Faculty of Education, Victoria University of Wellington

\section{Background}

For my Thesis towards a Master of Education, I am researching people volunteer, and how this is influenced by training for volunteers. Research has found volunteers' motivations vary and change, but there is not a lot of research into the impact of training on volunteers. Volunteers are really important for healthy communities, and so my hope is that this research might help to find out what is useful when training volunteers and also helps them to feel valued for the important work that they do.

\section{What I am asking of your organisation}

I would like to contact 5-10 new volunteers within your organisation and invite them to participate in this research. Participants will be asked to complete a short questionnaire and then take part in a 30-40 minute interview. This would take place at a time and place that is convenient to the participant. If required, a second interview would be arranged a number of months after the first so that I can see if motivations have changed at all since the first interview.

In addition to these interviews I would also like take a copy of any relevant documents that your organisation may keep in relation to volunteering. This could include such resources as recruitment and training materials, publicity documents, and volunteer entrance forms. After all of this data has been analysed, your organisation will be sent a summary of key themes so that you can let me know if you agree with them or not.

\section{What will happen to the information given?}

Participants will not be identified personally in any of the reports, nor will your organisation be identified directly. Some background around the nature of the volunteer work involved will be given however to provide context to the responses. Due to the small amount of volunteer organisations within the Wellington Region this may inadvertently imply your organisation to those who are familiar with your workplace. Note however that you will be made aware of any findings prior to publication, and the intention of the research is not to criticise, but rather to explore relationships between the motivations of volunteers and the training events you run.

All of the audio recordings and transcripts from interviews will be kept confidential, and viewed only by myself and my supervisor Stephanie Doyle (Senior Lecturer, School of Education). The finished thesis will be handed in for marking to the Faculty of Education, and then placed in the University Library. If you want to read the whole report at the end of my research, I am happy to make one available to you, and I would also be happy to provide a short presentation to your organisation on my research and findings. Reports on the 
research may also be published at academic conferences or in journals. All individual data collected from participants will be destroyed two years after the project is finished.

\section{What if I someone wants to pull out?}

Participation is entirely voluntary, and so anyone can elect to withdraw from the research up until the $30^{\text {th }}$ of September 2016. Any data collected about them would then be immediately destroyed.

\section{Contacts}

If you have any questions or would like to receive further information about the project, please contact me (dalyphil@myvuw.ac.nz), or my supervisor Dr Stephanie Doyle (Stephanie.Doyle@vuw.ac.nz or 04 463-6657). Victoria University requires, and has granted, approval from the University's Human Ethics Committee for this research. Should you have any concerns about the study, please contact the Convenor of the Human Ethics Committee, Associate Professor Susan Corbett (susan.corbett@vuw.ac.nz or 04463 5480).

Thank you very much for considering whether you want to take part in this project. I hope to talk to you soon.

Sincerely,

Phil Daly 


\section{Appendix B - Organisational Consent Form}

Research Project Title: Training for Volunteers - a multiple case study investigating the influence of volunteer motivations in two training programmes.

Researcher: Phil Daly, Faculty of Education, Victoria University of Wellington

I have had this research project explained to me, and understand what is required of individual participants and of my organisation.

I consent to the researcher interviewing members of my organisation for the purpose of this research, and to examine relevant organisational documents as outlined in the information sheet.

I have been given the opportunity to ask questions, and have been happy with the answers to these questions.

I understand that participation is voluntary, and that participants can withdraw from this project, without having to give reasons, by e-mailing dalyphil@myvuw.ac.nz by the $30^{\text {th }}$ of September 2016.

I understand that any information provided will be kept confidential to the researcher and their supervisor, the published results will not use individual participant's names, and that no opinions will be attributed in any way that will identify individual participants.

I understand that whilst my organisation will not be directly identified in the published results, as a result of the background information provided it may be able to be identified by those with knowledge of my workplace.

I understand that the data I provide will not be used for any other purpose or released to others, however findings may be published via academic conferences and/or journals.

I understand that the recording and transcripts of any interviews will be erased within two years after the conclusion of the project.

Please indicate (by ticking the boxes below) which of the following apply:

I would like to receive a summary of the results of this research when it is completed.

I agree to our volunteers being invited to participate in in this research.

I consent to provide the researchers with relevant documents about the recruitment and training of my our volunteers

Signed:

Name:

Job Title:

Date: 


\section{Appendix C - Participant Information Sheet}

Research Project Title: Training for Volunteers - a multiple case study investigating the influence of volunteer motivations in two training programmes.

Researcher: Phil Daly, Faculty of Education, Victoria University of Wellington

\section{Background}

For my Thesis towards a Master of Education, I am researching why people volunteer, and how training influences volunteering. Volunteers are really important for healthy communities, and so my hope is that this research might help to find out what is useful when training volunteers and also helps volunteers to feel valued for the important work that they do.

\section{What I am asking of you}

I am hoping that you might agree to take part in this research by completing a short questionnaire and begin interviewed once or twice. Each interview would take 30-40 minutes at a time and place that is easy for you. At the start of the interview I will ask if you are happy for me to make an audio recording of the interview, which I will later transcribe. If required, the second interview will take place a number of months after the first so that I can see if your motivations have changed at all since the first interview. After I have looked through all of the data I am going to make a summary of key themes, and send it out to you so that you can let me know if you agree with them or not.

What will happen to the information I give?

You will not be named in any of the reports, and I will be careful not to use any information that might identify you to others. The recordings and transcripts from interviews will be kept securely, and viewed only by myself and my supervisor Stephanie Doyle (Senior Lecturer, School of Education).

I will write my findings up as a thesis (report) and this will be handed in for marking to the Faculty of Education, and then placed in the University Library. If you want to read the whole report at the end of my research, I am happy to make one available to you. Reports on the research may also be published at academic conferences or in journals. All individual data collected from participants will be destroyed two years after the project is finished.

\section{What if you want to pull out?}

It is entirely up to you as to whether you want to take part in this research and you can pull out at any time up until the 30th of September 2016. Any data collected about you would then be immediately destroyed. 


\section{$\underline{\text { Contacts }}$}

If you have any questions or would like to receive further information about the project, please contact me (dalyphil@myvuw.ac.nz), or my supervisor Dr Stephanie Doyle (Stephanie.Doyle@vuw.ac.nz or 04 463-6657). Victoria University requires, and has granted, approval from the University's Human Ethics Committee for this research. Should you have any concerns about the study, please contact the Convenor of the Human Ethics Committee, Associate Professor Susan Corbett (susan.corbett@vuw.ac.nz or 04463 5480).

Thank you very much for considering whether you want to take part in this project. I hope to talk to you soon.

Sincerely,

Phil Daly 


\section{Appendix D - Participant Consent Form}

Research Project Title: Training for Volunteers - a multiple case study investigating the influence of volunteer motivations in two training programmes.

Researcher: Phil Daly, Faculty of Education, Victoria University of Wellington

I have had this research project explained to me, and understand what I am being asked to do.

I consent to complete a brief questionnaire, be interviewed by the researcher, and have this interview recorded for later transcription.

I have been given the opportunity to ask questions, and have been happy with the answers to these questions.

I understand that participation is voluntary, and that I can withdraw from this project, without having to give reasons, by e-mailing dalyphil@myvuw.ac.nz by the $30^{\text {th }}$ of September 2016.

I understand that any information I provide will be kept confidential to the researcher and their supervisor, the published results will not use my name, and that no opinions will be attributed to me in any way that will identify me.

I understand that the data I provide will not be used for any other purpose or released to others, however findings may be published via academic conferences and/or journals.

I understand that the recording and transcripts of the interviews will be erased within two years after the conclusion of the project.

Please indicate (by ticking the boxes below) which of the following apply:

I would like to receive a summary of the results of this research when it is completed.

I agree to this interview being audio recorded.

Signed:

Name of participant:

Date: 


\section{Appendix E - Interview Guide}

\section{Background info}

Gender:

Ethnicity:

Age Bracket:

Primary Occupation:

\section{Interview Questions}

How long have you volunteered with this organisation?

How would you describe your role with the organisation?

What were some of the main reasons for you to volunteer with ... when you first started?

Have these motivations shifted, or have you got any new motivations since volunteering with $\ldots$ ?

What were your initial thoughts towards the training event?

Did you see the training as a positive or negative for you, and why?

How would you describe the atmosphere at the training, and in particular the interactions between the tutor and the volunteers?

Did you feel that the training was relevant to you?

Were you allowed to make choices about your learning at the training?

How were your perspectives and values included into the training?

In what ways was the training challenging for you?

How did the training impact on you as a volunteer, and in terms of your volunteer role?

Were your reasons for wanting to volunteer helped, challenged, or shifted by what you learnt at the training?

Did the training relate at all to your reasons for volunteering?

Do you think that the training has inspired or equipped you to continue in your volunteering role?

Is there anything else that you would like to say which may be relevant to this research? 


\section{Appendix F - VFI Questionnaire}

Please indicate how important or accurate each of the 30 possible reasons for volunteering are for you in doing volunteer work. ( $1=$ not at all important/accurate; $7=$ extremely important/accurate.)

1. Volunteering can help me to get my foot in the door at a place where I would like to work.

$$
\begin{array}{llllllll}
1 & 2 & 3 & 4 & 5 & 6 & 7
\end{array}
$$

2. My friends volunteer.

$\begin{array}{lllllll}1 & 2 & 3 & 4 & 5 & 6 & 7\end{array}$

3. I am concerned about those less fortunate than myself.

$\begin{array}{llllllll}1 & 2 & 3 & 4 & 5 & 6 & 7\end{array}$

4. People I'm close to want me to volunteer.

$\begin{array}{lllllll}1 & 2 & 3 & 4 & 5 & 6 & 7\end{array}$

5. Volunteering makes me feel important.

$\begin{array}{lllllll}1 & 2 & 3 & 4 & 5 & 6 & 7\end{array}$

6. People I know share an interest in community service.

$\begin{array}{lllllll}1 & 2 & 3 & 4 & 5 & 6 & 7\end{array}$

7. No matter how bad I've been feeling, volunteering helps me to forget about it.

$\begin{array}{lllllll}1 & 2 & 3 & 4 & 5 & 6 & 7\end{array}$

8. I am genuinely concerned about the particular group I am serving.

$\begin{array}{lllllll}1 & 2 & 3 & 4 & 5 & 6 & 7\end{array}$

9. By volunteering I feel less lonely.

$\begin{array}{lllllll}1 & 2 & 3 & 4 & 5 & 6 & 7\end{array}$

10. I can make new contacts that might help my business or career.

$\begin{array}{lllllll}1 & 2 & 3 & 4 & 5 & 6 & 7\end{array}$

11. Doing volunteer work relieves me of some of the guilt over being more fortunate than others.

$$
\begin{array}{lllllll}
1 & 2 & 3 & 4 & 5 & 6 & 7
\end{array}
$$

12. I can learn more about the cause for which I am working.

$$
\begin{array}{llllllll}
1 & 2 & 3 & 4 & 5 & 6 & 7
\end{array}
$$

13. Volunteering increases my self-esteem.

$$
\begin{array}{lllllll}
1 & 2 & 3 & 4 & 5 & 6 & 7
\end{array}
$$

14. Volunteering allows me to gain a new perspective on things.

$$
\begin{array}{lllllll}
1 & 2 & 3 & 4 & 5 & 6 & 7
\end{array}
$$


15. Volunteering allows me to explore different career options.

$$
\begin{array}{lllllll}
1 & 2 & 3 & 4 & 5 & 6 & 7
\end{array}
$$

16. I feel compassion toward people in need.

$$
\begin{array}{llllllll}
1 & 2 & 3 & 4 & 5 & 6 & 7
\end{array}
$$

17. Others with whom I am close place a high value on community service.

$$
\begin{array}{lllllll}
1 & 2 & 3 & 4 & 5 & 6 & 7
\end{array}
$$

18. Volunteering lets me learn things through direct, hands on experience.

$$
\begin{array}{lllllll}
1 & 2 & 3 & 4 & 5 & 6 & 7
\end{array}
$$

19. I feel it is important to help others.

$$
\begin{array}{lllllll}
1 & 2 & 3 & 4 & 5 & 6 & 7
\end{array}
$$

20. Volunteering helps me work through my own personal problems.

$$
\begin{array}{lllllll}
1 & 2 & 3 & 4 & 5 & 6 & 7
\end{array}
$$

21. Volunteering will help me to succeed in my chosen profession.

$$
\begin{array}{lllllll}
1 & 2 & 3 & 4 & 5 & 6 & 7
\end{array}
$$

22. I can do something for a cause that is important to me.

$$
\begin{array}{lllllll}
1 & 2 & 3 & 4 & 5 & 6 & 7
\end{array}
$$

23. Volunteering is an important activity to the people I know best.

$$
\begin{array}{lllllll}
1 & 2 & 3 & 4 & 5 & 6 & 7
\end{array}
$$

24. Volunteering is a good escape from my own troubles.

$$
\begin{array}{llllllll}
1 & 2 & 3 & 4 & 5 & 6 & 7
\end{array}
$$

25. I can learn how to deal with a variety of people.

$$
\begin{array}{lllllll}
1 & 2 & 3 & 4 & 5 & 6 & 7
\end{array}
$$

26. Volunteering makes me feel needed.

$$
\begin{array}{lllllll}
1 & 2 & 3 & 4 & 5 & 6 & 7
\end{array}
$$

27. Volunteering makes me feel better about myself.

$$
\begin{array}{llllllll}
1 & 2 & 3 & 4 & 5 & 6 & 7
\end{array}
$$

28. Volunteering experience will look good on my resume

$$
\begin{array}{lllllll}
1 & 2 & 3 & 4 & 5 & 6 & 7
\end{array}
$$

29. Volunteering is a way to make new friends.

$$
\begin{array}{lllllll}
1 & 2 & 3 & 4 & 5 & 6 & 7
\end{array}
$$

30. I can explore my own strengths.

$\begin{array}{lllllll}1 & 2 & 3 & 4 & 5 & 6 & 7\end{array}$




\section{Appendix G - VFI Questions by Motivational Function}

VFI Questions (re-ordered by motivational function)

\section{Career}

Volunteering can help me to get my foot in the door at a place where I would like to work.

I can make new contacts that might help my business or career.

Volunteering allows me to explore different career options.

Volunteering will help me to succeed in my chosen profession.

Volunteering experience will look good on my resume.

\section{Social}

My friends volunteer.

People I'm close to want me to volunteer.

People I know share an interest in community service.

Others with whom I am close place a high value on community service.

Volunteering is an important activity to the people I know best.

\section{Values}

I am concerned about those less fortunate than myself.

I am genuinely concerned about the particular group I am serving.

I feel compassion toward people in need.

I feel it is important to help others.

I can do something for a cause that is important to me.

\section{Enhancement}

Volunteering makes me feel important.

Volunteering increases my self-esteem.

Volunteering makes me feel needed.

Volunteering makes me feel better about myself.

Volunteering is a way to make new friends. 


\section{Protection}

No matter how bad I've been feeling, volunteering helps me to forget about it.

By volunteering I feel less lonely.

Doing volunteer work relieves me of some of the guilt over being more fortunate than others.

Volunteering helps me work through my own personal problems.

Volunteering is a good escape from my own troubles.

\section{Understanding}

I can learn more about the cause for which I am working.

Volunteering allows me to gain a new perspective on things.

Volunteering lets me learn things through direct, hands on experience.

I can learn how to deal with a variety of people.

I can explore my own strengths. 